\title{
Rhodium-Catalyzed Dehydrogenative Annulation of \\ $N$-Arylmethanimines with Vinylene Carbonate for Synthesizing \\ Quinolines
}

\begin{abstract}
Yan $\mathrm{Hu}$, Jiang Nan,* Jiacheng Yin, Guanjie Huang, Xin Ren, Yangmin Ma*
Key Laboratory of Chemical Additives for China National Light Industry, College of Chemistry and Chemical Engineering, Shaanxi University of Science and Technology, Xi'an 710021, China
\end{abstract}

Email: nanjiang@sust.edu.cn

E-mail: mym63@sina.com.

\section{Supporting Information}

Table of Contents:

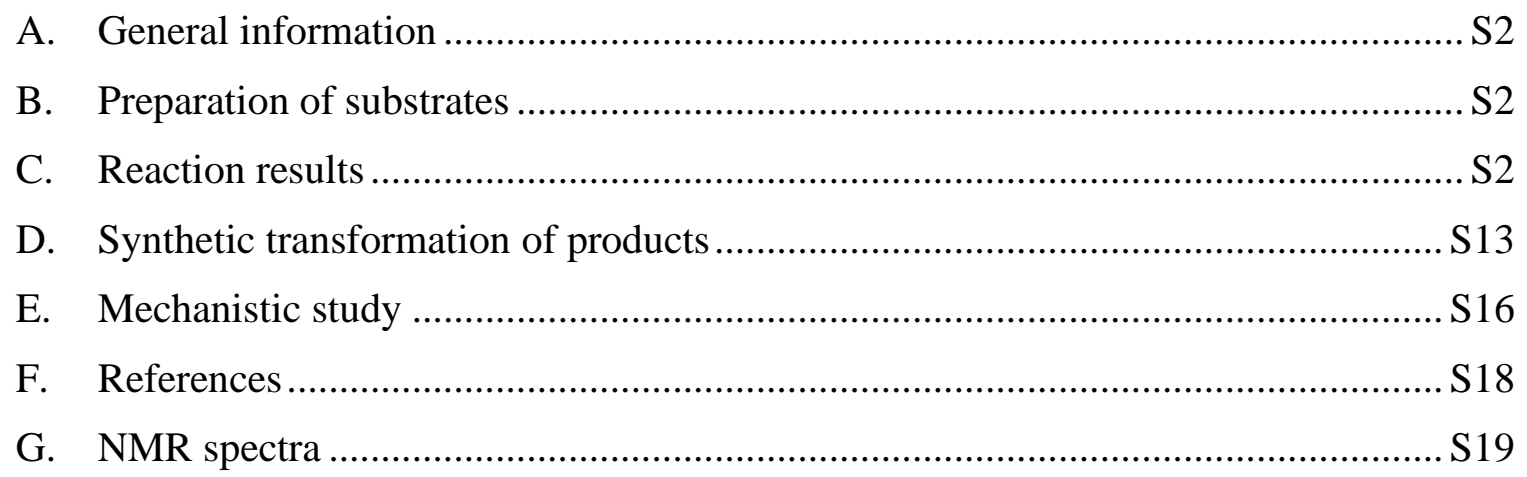




\section{A. General information}

All reagents were used as received unless otherwise noted. The vinylene carbonate $\mathbf{2}$, amines 4 and aldehydes 5 used for experiments were purchased from Aladdin and Sigma-Aldrich. Analytical thin-layer chromatography was performed with $0.25 \mathrm{~mm}$ coated commercial silica gel plates (TLC Silica Gel $60 \mathrm{~F}_{254}$ ); visualization of the developed chromatogram was performed by fluorescence. Flash Chromatography was performed with silica gel (300-400 mesh). Proton-1 nuclear magnetic resonance ( ${ }^{1} \mathrm{H}$ NMR) data were acquired at $400 \mathrm{MHz}$ on a Bruker Ascend 400 $(400 \mathrm{MHz})$ spectrometer, and chemical shifts are reported in delta $(\delta)$ units, in parts per million (ppm) downfield from tetramethylsilane. Splitting patterns are designated as s, singlet; d, doublet; t, triplet; q, quartet; m, multiplet, coupling constants $J$ are quoted in Hz. Carbon-13 nuclear magnetic resonance $\left({ }^{13} \mathrm{C} \mathrm{NMR}\right)$ data were acquired at $100 \mathrm{MHz}$ on a Bruker Ascend 400 spectrometer, chemical shifts are reported in ppm relative to the center line of a triplet at $77.0 \mathrm{ppm}$ for $\mathrm{CDCl}_{3}$. Infrared spectra (IR) data were recorded on a TENSOR 27 FT-IR spectrometer and recorded in wave numbers $\left(\mathrm{cm}^{-1}\right)$. High resolution mass spectra were acquired on a Bruker Daltonics MicroTof-Q II mass spectrometer. $\mathbf{1 a}^{\mathbf{1}}, \mathbf{1 b}-\mathbf{1 f}^{\mathbf{2}}, \mathbf{1 g}^{\mathbf{1}}, \mathbf{1 h}-\mathbf{1 l}^{\mathbf{3}}, \mathbf{1 m}-\mathbf{1 r}^{\mathbf{4}}, \mathbf{1} \mathbf{s}^{\mathbf{5}}, \mathbf{1 t}-\mathbf{1 u}^{\mathbf{6}}$ were prepared according to literature methods.

\section{B. Preparation of substrates}

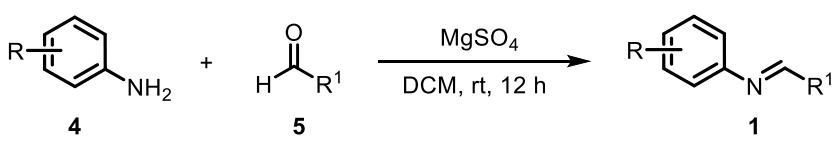

A round bottom flask equipped with stir bar was charged with amines $4(3.0 \mathrm{mmol})$, aldehydes $5(3.0 \mathrm{mmol})$ and anhydrous DCM $(8.0 \mathrm{~mL})$, followed by addition of anhydrous $\mathrm{MgSO}_{4}$ ( $6.0 \mathrm{mmol})$. The mixture was stirred at room temperature overnight. After filtration of $\mathrm{MgSO}_{4}$, The filtrate was concentrated under reduced pressure, and the crude product was purified by column chromatography to obtain the corresponding imines $\mathbf{1}$.

\section{Reaction results}

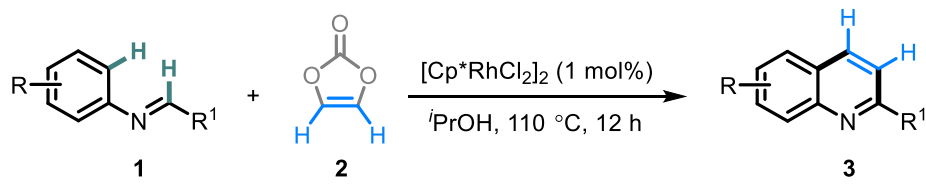

A pressure tube was charged with imines $1(0.30 \mathrm{mmol})$, vinylene carbonate $2(0.20 \mathrm{mmol})$ $\left[\mathrm{Cp} * \mathrm{RhCl}_{2}\right]_{2}(1.2 \mathrm{mg}, 0.002 \mathrm{mmol})$, and ${ }^{i} \mathrm{PrOH}(2.5 \mathrm{~mL})$. The reaction mixture was stirred at $110{ }^{\circ} \mathrm{C}$ for $12 \mathrm{~h}$ under Ar atmosphere in an oil bath. After cooling to room temperature, all volatiles were removed under reduced pressure. The residue was purified by silica gel chromatography using PE/EA eluent to afford the product 3 .

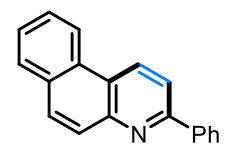

\section{3-Phenylbenzo[f]quinoline (3a)}

White solid (34.7 mg, 68\% yield). PE/EA $=30: 1, \mathrm{R}_{\mathrm{f}}=0.28 . \mathrm{mp}: 179-181{ }^{\circ} \mathrm{C} .{ }^{1} \mathrm{H}$ NMR $(400$ $\left.\mathrm{MHz}, \mathrm{CDCl}_{3}\right): \delta 9.01(\mathrm{~d}, J=8.7 \mathrm{~Hz}, 1 \mathrm{H}), 8.66(\mathrm{~d}, J=8.0 \mathrm{~Hz}, 1 \mathrm{H}), 8.26(\mathrm{~d}, J=7.5 \mathrm{~Hz}, 2 \mathrm{H}), 8.13$ $(\mathrm{d}, J=9.2 \mathrm{~Hz}, 1 \mathrm{H}), 8.09-8.01(\mathrm{~m}, 2 \mathrm{H}), 7.99(\mathrm{~d}, J=7.9 \mathrm{~Hz}, 1 \mathrm{H}), 7.78-7.65(\mathrm{~m}, 2 \mathrm{H}), 7.64-7.48(\mathrm{~m}$, 
3H). ${ }^{13} \mathrm{C}$ NMR (100 MHz, $\left.\mathrm{CDCl}_{3}\right): \delta 156.9,148.2,139.5,131.7,131.5,131.0,129.7,129.3,128.9$, 128.7, 128.7, 127.5, 127.1, 127.1, 124.2, 122.7, 118.8. IR (KBr): 3109, 1641, 1258, 1125, 832, $748,685 \mathrm{~cm}^{-1}$. HRMS (ESI) m/z Calcd for $\mathrm{C}_{19} \mathrm{H}_{14} \mathrm{~N}[\mathrm{M}+\mathrm{H}]^{+} 256.1126$, found 256.1123 .

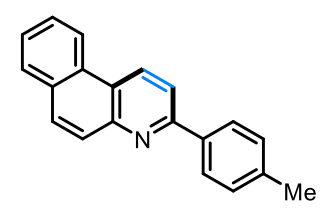

\section{3-(p-Tolyl)benzo[f]quinoline (3b)}

White solid (38.8 mg, 72\% yield). PE/EA $=30: 1, \mathrm{R}_{\mathrm{f}}=0.26 . \mathrm{mp}: 138-140{ }^{\circ} \mathrm{C} .{ }^{1} \mathrm{H}$ NMR $(400$ $\left.\mathrm{MHz}, \mathrm{CDCl}_{3}\right): \delta 8.98(\mathrm{~d}, J=8.7 \mathrm{~Hz}, 1 \mathrm{H}), 8.65(\mathrm{~d}, J=7.9 \mathrm{~Hz}, 1 \mathrm{H}), 8.21-8.10(\mathrm{~m}, 3 \mathrm{H}), 8.08-7.95$ (m, 3H), 7.77-7.65 (m, 2H), $7.40(\mathrm{~d}, J=7.9 \mathrm{~Hz}, 2 \mathrm{H}), 2.50(\mathrm{~s}, 3 \mathrm{H}) .{ }^{13} \mathrm{C} \mathrm{NMR}\left(100 \mathrm{MHz}, \mathrm{CDCl}_{3}\right)$ : $\delta 156.9,148.2,139.3,136.6,131.6,131.4,130.9,129.7,129.6,128.7,128.6,127.3,127.0,127.0$, 124.0, 122.6, 118.6, 21.4. IR (KBr): 3021, 1635, 1493, 1370, 1203, 879, 816, $746 \mathrm{~cm}^{-1}$. HRMS (ESI) $\mathrm{m} / \mathrm{z}$ Calcd for $\mathrm{C}_{20} \mathrm{H}_{16} \mathrm{~N}[\mathrm{M}+\mathrm{H}]^{+} 270.1283$, found 270.1281 .

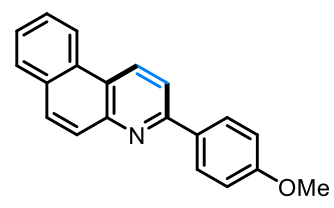

\section{3-(4-Methoxyphenyl)benzo[ $f]$ quinoline (3c)}

White solid (42.8 mg, 75\% yield). PE/EA $=30: 1, \mathrm{R}_{\mathrm{f}}=0.21 . \mathrm{mp}: 155-157{ }^{\circ} \mathrm{C} .{ }^{1} \mathrm{H}$ NMR $(400$ $\left.\mathrm{MHz}, \mathrm{CDCl}_{3}\right): \delta 9.00(\mathrm{~d}, J=8.7 \mathrm{~Hz}, 1 \mathrm{H}), 8.66(\mathrm{~d}, J=8.1 \mathrm{~Hz}, 1 \mathrm{H}), 8.23(\mathrm{~d}, J=8.9 \mathrm{~Hz}, 2 \mathrm{H}), 8.10$ (d, $J=9.1 \mathrm{~Hz}, 1 \mathrm{H}), 8.07-7.94(\mathrm{~m}, 3 \mathrm{H}), 7.78-7.63(\mathrm{~m}, 2 \mathrm{H}), 7.11(\mathrm{~d}, J=8.8 \mathrm{~Hz}, 2 \mathrm{H}), 3.95(\mathrm{~s}, 3 \mathrm{H})$. ${ }^{13} \mathrm{C}$ NMR $\left(100 \mathrm{MHz}, \mathrm{CDCl}_{3}\right): \delta 160.8,156.5,148.2,132.1,131.6,131.4,130.9,129.7,128.7$, 128.7, 128.6, 127.0, 126.9, 123.7, 122.5, 118.3, 114.3, 55.4. IR (KBr): 2987, 1599, 1467, 1253, 1179, 1026, 824, $748 \mathrm{~cm}^{-1}$. HRMS (ESI) m/z Calcd for $\mathrm{C}_{20} \mathrm{H}_{16} \mathrm{NO}[\mathrm{M}+\mathrm{H}]^{+}$286.1232, found 286.1229 .

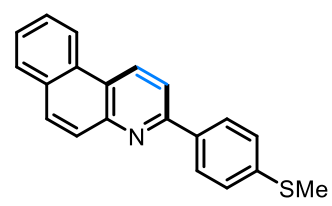

\section{3-(4-(Methylthio)phenyl)benzo[f]quinoline (3d)}

White solid (44.6 mg, 74\% yield). PE/EA $=30: 1, R_{\mathrm{f}}=0.22 . \mathrm{mp}: 201-203{ }^{\circ} \mathrm{C} .{ }^{1} \mathrm{H}$ NMR (400 $\left.\mathrm{MHz}, \mathrm{CDCl}_{3}\right): \delta 9.03(\mathrm{~d}, J=8.7 \mathrm{~Hz}, 1 \mathrm{H}), 8.67(\mathrm{~d}, J=8.2 \mathrm{~Hz}, 1 \mathrm{H}), 8.22(\mathrm{~d}, J=7.8 \mathrm{~Hz}, 2 \mathrm{H}), 8.12$ (d, $J=9.1 \mathrm{~Hz}, 1 \mathrm{H}), 8.08-7.97$ (m, 3H), 7.80-7.68 (m, 2H), $7.46(\mathrm{~d}, J=7.3 \mathrm{~Hz}, 2 \mathrm{H}), 2.62(\mathrm{~s}, 3 \mathrm{H})$. ${ }^{13} \mathrm{C}$ NMR (100 MHz, $\mathrm{CDCl}_{3}$ ): $\delta 156.2,148.2,140.3,136.1,131.7,131.5,131.0,129.7,128.7$, 128.6, 127.7, 127.1, 126.5, 124.1, 122.6, 118.3, 15.6. IR (KBr): 1659, 1473, 1321, 1090, 989, 814 , $744 \mathrm{~cm}^{-1}$. HRMS (ESI) m/z Calcd for $\mathrm{C}_{20} \mathrm{H}_{16} \mathrm{NS}[\mathrm{M}+\mathrm{H}]^{+} 302.1003$, found 302.1003 .

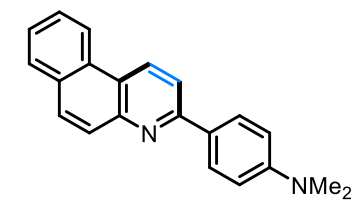

\section{4-(Benzo[f]quinolin-3-yl)-N,N-dimethylaniline (3e)}


Yellow solid (33.4 mg, 56\% yield). PE/EA $=20: 1, \mathrm{R}_{\mathrm{f}}=0.20 . \mathrm{mp}: 207-209^{\circ} \mathrm{C} .{ }^{1} \mathrm{H}$ NMR $(400$ MHz, $\left.\mathrm{CDCl}_{3}\right): \delta 8.96(\mathrm{~d}, J=8.7 \mathrm{~Hz}, 1 \mathrm{H}), 8.64(\mathrm{~d}, J=8.2 \mathrm{~Hz}, 1 \mathrm{H}), 8.21(\mathrm{~d}, J=8.5 \mathrm{~Hz}, 2 \mathrm{H}), 8.09$ (d, $J=9.1 \mathrm{~Hz}, 1 \mathrm{H}), 8.05-7.93(\mathrm{~m}, 3 \mathrm{H}), 7.76-7.61(\mathrm{~m}, 2 \mathrm{H}), 6.90(\mathrm{~d}, J=8.8 \mathrm{~Hz}, 2 \mathrm{H}), 3.10(\mathrm{~s}, 6 \mathrm{H})$. ${ }^{13} \mathrm{C}$ NMR (100 MHz, $\left.\mathrm{CDCl}_{3}\right): \delta 157.0,151.4,148.2,131.4,131.1,130.6,129.9,128.7,128.6$, 128.3, 127.2, 126.9, 126.6, 123.2, 122.4, 117.9, 112.3, 40.4. IR (KBr): 3126, 1644, 1598, 1503, 1363, 1135, 818, $748 \mathrm{~cm}^{-1}$. HRMS (ESI) $\mathrm{m} / \mathrm{z}$ Calcd for $\mathrm{C}_{21} \mathrm{H}_{19} \mathrm{~N}_{2}[\mathrm{M}+\mathrm{H}]^{+} 299.1548$, found 299.1545.

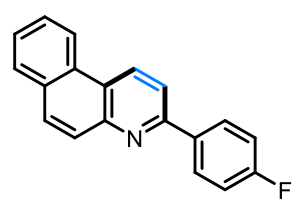

\section{3-(4-Fluorophenyl)benzo[f]quinoline (3f)}

White solid (38.8 mg, 71\% yield). PE/EA $=30: 1, \mathrm{R}_{\mathrm{f}}=0.33 . \mathrm{mp}: 157-159{ }^{\circ} \mathrm{C} .{ }^{1} \mathrm{H}$ NMR $(400$ $\left.\mathrm{MHz}, \mathrm{CDCl}_{3}\right): \delta 8.97(\mathrm{~d}, J=8.7 \mathrm{~Hz}, 1 \mathrm{H}), 8.63(\mathrm{~d}, J=7.4 \mathrm{~Hz}, 1 \mathrm{H}), 8.28-8.19(\mathrm{~m}, 2 \mathrm{H}), 8.10(\mathrm{~d}, J=$ $9.1 \mathrm{~Hz}, 1 \mathrm{H}), 8.04(\mathrm{~d}, J=9.1 \mathrm{~Hz}, 1 \mathrm{H}), 8.01-7.93(\mathrm{~m}, 2 \mathrm{H}), 7.79-7.64(\mathrm{~m}, 2 \mathrm{H}), 7.26(\mathrm{t}, J=8.7 \mathrm{~Hz}$, 2H). ${ }^{13} \mathrm{C}$ NMR (100 MHz, $\left.\mathrm{CDCl}_{3}\right): \delta 163.5(\mathrm{~d}, J=247.4 \mathrm{~Hz}), 155.7,148.2,135.6(\mathrm{~d}, J=2.8 \mathrm{~Hz})$, 131.7, 131.6, 131.1, 129.6, 129.3, 129.2, 128.6 (d, $J=24.7 \mathrm{~Hz}), 127.2$, 127.1, 124.1, 122.6, 118.4, $115.8(\mathrm{~d}, J=21.4 \mathrm{~Hz}) .{ }^{19} \mathrm{~F}$ NMR $\left(376 \mathrm{MHz}, \mathrm{CDCl}_{3}\right): \delta-112.44$. IR (KBr): 3029, 1644, 1466, 1124 , $1019,822,745 \mathrm{~cm}^{-1}$. HRMS (ESI) m/z Calcd for $\mathrm{C}_{19} \mathrm{H}_{13} \mathrm{FN}[\mathrm{M}+\mathrm{H}]^{+} 274.1032$, found 274.1028.

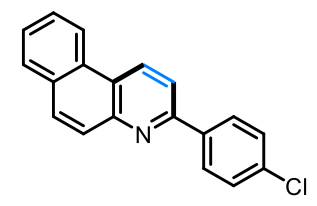

\section{3-(4-Chlorophenyl)benzo[f $f$ quinoline (3g)}

White solid (39.8 mg, 69\% yield). PE/EA $=30: 1, \mathrm{R}_{\mathrm{f}}=0.29 . \mathrm{mp}: 178-180{ }^{\circ} \mathrm{C} .{ }^{1} \mathrm{H}$ NMR $(400$ $\left.\mathrm{MHz}, \mathrm{CDCl}_{3}\right): \delta 9.03(\mathrm{~d}, J=8.7 \mathrm{~Hz}, 1 \mathrm{H}), 8.67(\mathrm{~d}, J=8.3 \mathrm{~Hz}, 1 \mathrm{H}), 8.21(\mathrm{~d}, J=8.3 \mathrm{~Hz}, 2 \mathrm{H})$, 8.13-7.95 (m, 4H), 7.80-7.66 (m, 2H), $7.56(\mathrm{~d}, J=8.3 \mathrm{~Hz}, 2 \mathrm{H}) .{ }^{13} \mathrm{C} \mathrm{NMR}\left(100 \mathrm{MHz}, \mathrm{CDCl}_{3}\right): \delta$ 155.5, 148.2, 137.8, 135.4, 131.7, 131.6, 131.2, 129.5, 129.0, 128.7, 128.6, 128.5, 127.2, 127.2, 124.3, 122.6, 118.4. IR (KBr): 3016, 1643, 1423, 1202, 1097, 822, $749 \mathrm{~cm}^{-1}$. HRMS (ESI) m/z Calcd for $\mathrm{C}_{19} \mathrm{H}_{13} \mathrm{ClN}[\mathrm{M}+\mathrm{H}]^{+} 290.0737$, found 290.0734 .

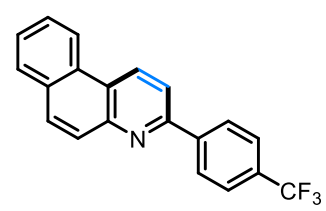

\section{3-(4-(Trifluoromethyl)phenyl)benzo[f]quinoline (3h)}

White solid (42.1 mg, 65\% yield). PE/EA $=30: 1, \mathrm{R}_{\mathrm{f}}=0.32 . \mathrm{mp}: 163-165{ }^{\circ} \mathrm{C} .{ }^{1} \mathrm{H}$ NMR $(400$ $\left.\mathrm{MHz}, \mathrm{CDCl}_{3}\right): \delta 9.07(\mathrm{~d}, J=8.7 \mathrm{~Hz}, 1 \mathrm{H}), 8.68(\mathrm{~d}, J=8.8 \mathrm{~Hz}, 1 \mathrm{H}), 8.37(\mathrm{~d}, J=8.1 \mathrm{~Hz}, 2 \mathrm{H})$, 8.15-8.04 (m, 3H), $8.00(\mathrm{~d}, J=7.7 \mathrm{~Hz}, 1 \mathrm{H}), 7.84(\mathrm{~d}, J=8.1 \mathrm{~Hz}, 2 \mathrm{H}), 7.79-7.69(\mathrm{~m}, 2 \mathrm{H}) .{ }^{13} \mathrm{C}$ NMR (100 MHz, $\left.\mathrm{CDCl}_{3}\right): \delta 155.1,148.3,142.7,131.8,131.8,131.4,131.0$ (q, $\left.J=32.3 \mathrm{~Hz}\right), 129.5$, 128.8, 128.5, 127.7, 127.5, 127.3, $125.8(\mathrm{q}, J=3.2 \mathrm{~Hz}), 124.7,124.2(\mathrm{q}, J=270 \mathrm{~Hz}), 122.8$, 118.7. 
${ }^{19} \mathrm{~F}$ NMR (376 MHz, $\left.\mathrm{CDCl}_{3}\right): \delta$-62.49. IR (KBr): 2987, 1622, 1511, 1269, 1121, 826, $753 \mathrm{~cm}^{-1}$. HRMS (ESI) m/z Calcd for $\mathrm{C}_{20} \mathrm{H}_{13} \mathrm{~F}_{3} \mathrm{~N}[\mathrm{M}+\mathrm{H}]^{+} 324.1000$, found 324.0999 .

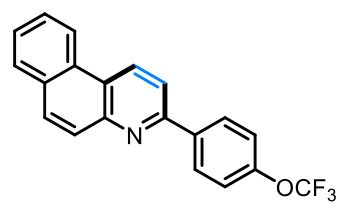

\section{3-(4-(Trifluoromethoxy)phenyl)benzo[f]quinoline (3i)}

White solid (35.9 mg, 53\% yield). PE/EA $=50: 1, \mathrm{R}_{\mathrm{f}}=0.25 . \mathrm{mp}: 182-184{ }^{\circ} \mathrm{C} .{ }^{1} \mathrm{H}$ NMR $(400$ $\left.\mathrm{MHz}, \mathrm{CDCl}_{3}\right): \delta 9.05(\mathrm{~d}, J=8.6 \mathrm{~Hz}, 1 \mathrm{H}), 8.68(\mathrm{~d}, J=8.0 \mathrm{~Hz}, 1 \mathrm{H}), 8.30(\mathrm{~d}, J=8.2 \mathrm{~Hz}, 2 \mathrm{H})$, 8.15-7.97 (m, 4H), 7.80-7.68 (m, 2H), $7.43(\mathrm{~d}, J=8.2 \mathrm{~Hz}, 2 \mathrm{H}) .{ }^{13} \mathrm{C} \mathrm{NMR}\left(100 \mathrm{MHz}, \mathrm{CDCl}_{3}\right): \delta$ $155.3,150.1,148.2,138.1,131.7,131.7,131.3,129.5,128.9,128.8,128.5,127.3,127.2,124.3$, 122.7, 121.2, $120.1(\mathrm{q}, J=255.8 \mathrm{~Hz}), 118.5 .{ }^{19} \mathrm{~F} \mathrm{NMR}\left(376 \mathrm{MHz}, \mathrm{CDCl}_{3}\right): \delta-57.65 . \mathrm{IR}(\mathrm{KBr}):$ 3087, 1632, 1476, 1326, 1238, 1159, 817, $752 \mathrm{~cm}^{-1}$. HRMS (ESI) $\mathrm{m} / \mathrm{z}$ Calcd for $\mathrm{C}_{20} \mathrm{H}_{13} \mathrm{~F}_{3} \mathrm{NO}$ $[\mathrm{M}+\mathrm{H}]^{+}$340.0949, found 340.0945 .

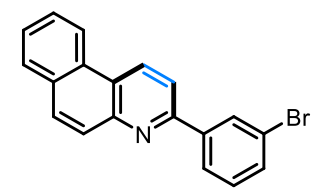

\section{3-(3-Bromophenyl)benzo[f $f$ quinoline (3j)}

White solid (44.8 mg, 67\% yield). PE/EA $=30: 1, \mathrm{R}_{\mathrm{f}}=0.31 . \mathrm{mp}: 106-108{ }^{\circ} \mathrm{C} .{ }^{1} \mathrm{H}$ NMR $(400$ MHz, $\left.\mathrm{CDCl}_{3}\right): \delta 9.05(\mathrm{~d}, J=8.6 \mathrm{~Hz}, 1 \mathrm{H}), 8.68(\mathrm{~d}, J=7.9 \mathrm{~Hz}, 1 \mathrm{H}), 8.45(\mathrm{~s}, 1 \mathrm{H}), 8.17(\mathrm{~d}, J=7.8$ $\mathrm{Hz}, 1 \mathrm{H}), 8.14-7.96(\mathrm{~m}, 4 \mathrm{H}), 7.80-7.67(\mathrm{~m}, 2 \mathrm{H}), 7.64(\mathrm{~d}, J=7.9 \mathrm{~Hz}, 1 \mathrm{H}), 7.45(\mathrm{t}, J=7.9 \mathrm{~Hz}, 1 \mathrm{H})$. ${ }^{13} \mathrm{C}$ NMR $\left(100 \mathrm{MHz}, \mathrm{CDCl}_{3}\right): \delta 155.1,148.2,141.5,132.2,131.8,131.7,131.3,130.5,130.4$, 129.5, 128.8, 128.5, 127.4, 127.2, 125.9, 124.5, 123.2, 122.7, 118.6. IR (KBr): 3058, 1643, 1477, 1273, 1079, 807, $738 \mathrm{~cm}^{-1}$. HRMS (ESI) $\mathrm{m} / \mathrm{z}$ Calcd for $\mathrm{C}_{19} \mathrm{H}_{13} \mathrm{BrN}[\mathrm{M}+\mathrm{H}]^{+} 334.0231$, found 334.0228 .

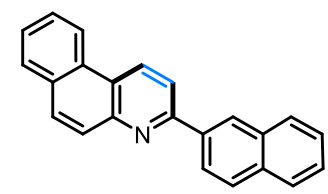

3-(Naphthalen-2-yl)benzo[f]quinoline (3k)

White solid (38.5 mg, 63\% yield). PE/EA $=30: 1, \mathrm{R}_{\mathrm{f}}=0.26 . \mathrm{mp}: 206-208{ }^{\circ} \mathrm{C} .{ }^{1} \mathrm{H}$ NMR $(400$ $\left.\mathrm{MHz}, \mathrm{CDCl}_{3}\right): \delta 9.07(\mathrm{~d}, J=8.7 \mathrm{~Hz}, 1 \mathrm{H}), 8.76-8.65(\mathrm{~m}, 2 \mathrm{H}), 8.45(\mathrm{~d}, J=8.6 \mathrm{~Hz}, 1 \mathrm{H}), 8.26-8.14$ (m, 2H), 8.11-7.92 (m, 5H), 7.80-7.66 (m, 2H), 7.63-7.53 (m, 2H). $\left.{ }^{13} \mathrm{C} \mathrm{NMR} \mathrm{(100} \mathrm{MHz,} \mathrm{CDCl}_{3}\right)$ : $\delta 156.7,148.4,136.8,133.9,133.6,131.7,131.5,131.1,129.7,128.9,128.8,128.7,128.6,127.7$, 127.2, 127.1, 127.0, 126.7, 126.3, 125.0, 124.2, 122.7, 119.0. IR (KBr): 3060, 1702, 1643, 1568, $1167,822,740 \mathrm{~cm}^{-1}$. HRMS (ESI) m/z Calcd for $\mathrm{C}_{23} \mathrm{H}_{16} \mathrm{~N}[\mathrm{M}+\mathrm{H}]^{+} 306.1283$, found 306.1281 .

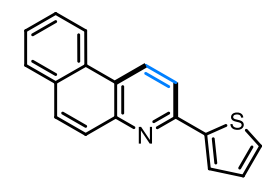


White solid (30.3 mg, 58\% yield). PE/EA $=30: 1, \mathrm{R}_{\mathrm{f}}=0.28 . \mathrm{mp}: 176-178{ }^{\circ} \mathrm{C} .{ }^{1} \mathrm{H}$ NMR $(400$ $\left.\mathrm{MHz}, \mathrm{CDCl}_{3}\right): \delta 8.95(\mathrm{~d}, J=8.7 \mathrm{~Hz}, 1 \mathrm{H}), 8.62(\mathrm{~d}, J=8.1 \mathrm{~Hz}, 1 \mathrm{H}), 8.08-8.00(\mathrm{~m}, 2 \mathrm{H}), 7.97(\mathrm{~d}, J=$ $8.6 \mathrm{~Hz}, 2 \mathrm{H}), 7.81(\mathrm{~d}, J=3.7 \mathrm{~Hz}, 1 \mathrm{H}), 7.77-7.64(\mathrm{~m}, 2 \mathrm{H}), 7.52(\mathrm{~d}, J=5.1 \mathrm{~Hz}, 1 \mathrm{H}), 7.25-7.19(\mathrm{~m}$, 1H). ${ }^{13} \mathrm{C}$ NMR (100 MHz, $\left.\mathrm{CDCl}_{3}\right): \delta 152.0,148.1,145.2,131.6,131.4,131.1,129.6,128.7,128.3$, 128.2, 128.1, 127.1, 127.0, 125.6, 124.1, 122.5, 117.4. IR (KBr): 3087, 1644, 1488, 1253, 1060 , $926,830,675 \mathrm{~cm}^{-1}$. HRMS (ESI) $\mathrm{m} / \mathrm{z}$ Calcd for $\mathrm{C}_{17} \mathrm{H}_{12} \mathrm{NS}[\mathrm{M}+\mathrm{H}]^{+} 262.0690$, found 262.0688 .

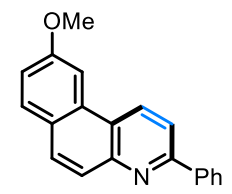

\section{9-Methoxy-3-phenylbenzo[f]quinoline (3m)}

White solid (45.6 mg, 80\% yield). PE/EA $=30: 1, \mathrm{R}_{\mathrm{f}}=0.19 . \mathrm{mp}: 181-183{ }^{\circ} \mathrm{C} .{ }^{1} \mathrm{H}$ NMR $(400$ $\left.\mathrm{MHz}, \mathrm{CDCl}_{3}\right): \delta 8.93(\mathrm{~d}, J=8.6 \mathrm{~Hz}, 1 \mathrm{H}), 8.29-8.21(\mathrm{~m}, 2 \mathrm{H}), 8.04-7.94(\mathrm{~m}, 4 \mathrm{H}), 7.89(\mathrm{~d}, J=8.7$ $\mathrm{Hz}, 1 \mathrm{H}), 7.63-7.56(\mathrm{~m}, 2 \mathrm{H}), 7.55-7.48(\mathrm{~m}, 1 \mathrm{H}), 7.34-7.30(\mathrm{~m}, 1 \mathrm{H}), 4.07(\mathrm{~s}, 3 \mathrm{H}) .{ }^{13} \mathrm{C}$ NMR $(100$ $\left.\mathrm{MHz}, \mathrm{CDCl}_{3}\right): \delta 158.9,156.9,148.6,139.5,131.5,131.1,130.7,130.1,129.3,128.9,127.5,126.4$, 126.2, 123.6, 118.3, 117.2, 104.0, 55.5. IR (KBr): 3017, 1618, 1494, 1226, 1031, 836, 756, 686 $\mathrm{cm}^{-1}$. HRMS (ESI) $\mathrm{m} / \mathrm{z}$ Calcd for $\mathrm{C}_{20} \mathrm{H}_{16} \mathrm{NO}[\mathrm{M}+\mathrm{H}]^{+} 286.1232$, found 286.1228 .

\section{8-Methyl-3-phenylbenzo[f]quinoline (3n)}

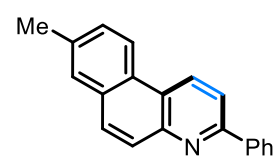

White solid (42.0 mg, 78\% yield). PE/EA = 30:1, $\mathrm{R}_{\mathrm{f}}=0.30 . \mathrm{mp}: 145-147{ }^{\circ} \mathrm{C} .{ }^{1} \mathrm{H}$ NMR (400 $\left.\mathrm{MHz}, \mathrm{CDCl}_{3}\right): \delta 8.95(\mathrm{~d}, J=8.7 \mathrm{~Hz}, 1 \mathrm{H}), 8.52(\mathrm{~d}, J=8.4 \mathrm{~Hz}, 1 \mathrm{H}), 8.30-8.22(\mathrm{~m}, 2 \mathrm{H}), 8.10(\mathrm{~d}, J=$ $9.0 \mathrm{~Hz}, 1 \mathrm{H}), 7.99(\mathrm{~d}, J=13.0 \mathrm{~Hz}, 2 \mathrm{H}), 7.76(\mathrm{~s}, 1 \mathrm{H}), 7.64-7.48(\mathrm{~m}, 4 \mathrm{H}), 2.63(\mathrm{~s}, 3 \mathrm{H}) .{ }^{13} \mathrm{C}$ NMR $\left(100 \mathrm{MHz}, \mathrm{CDCl}_{3}\right): \delta 156.4,148.0,139.6,137.0,131.9,131.2,130.7,129.1,128.8,128.6,128.3$, 127.5, 127.4, 124.2, 122.5, 118.7, 21.5. IR (KBr): 3087, 1639, 1486, 1263, 1054, 887, $684 \mathrm{~cm}^{-1}$. HRMS (ESI) m/z Calcd for $\mathrm{C}_{20} \mathrm{H}_{16} \mathrm{~N}[\mathrm{M}+\mathrm{H}]^{+} 270.1283$, found 270.1281 .

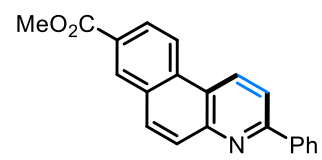

\section{Methyl 3-phenylbenzo[f]quinoline-8-carboxylate (3o)}

White solid (38.9 mg, 62\% yield). PE/EA $=20: 1, R_{\mathrm{f}}=0.19 . \mathrm{mp}: 156-158{ }^{\circ} \mathrm{C} .{ }^{1} \mathrm{H}$ NMR $(400$ $\left.\mathrm{MHz}, \mathrm{CDCl}_{3}\right): \delta 8.99(\mathrm{~d}, J=8.7 \mathrm{~Hz}, 1 \mathrm{H}), 8.70-8.60(\mathrm{~m}, 2 \mathrm{H}), 8.31(\mathrm{~d}, J=8.5 \mathrm{~Hz}, 1 \mathrm{H}), 8.26(\mathrm{~d}, J=$ $7.4 \mathrm{~Hz}, 2 \mathrm{H}), 8.17-8.12(\mathrm{~m}, 1 \mathrm{H}), 8.11-8.02(\mathrm{~m}, 2 \mathrm{H}), 7.63-7.49(\mathrm{~m}, 3 \mathrm{H}), 4.06(\mathrm{~s}, 3 \mathrm{H}) .{ }^{13} \mathrm{C}$ NMR $\left(100 \mathrm{MHz}, \mathrm{CDCl}_{3}\right): \delta 166.9,157.8,149.0,139.1,132.7,132.0,131.2,131.0,130.9,129.6,129.5$, 128.9, 128.5, 127.5, 126.9, 123.6, 122.9, 119.1, 52.4. IR (KBr): 2987, 1714, 1642, 1272, 1080 , 830, 761, $685 \mathrm{~cm}^{-1}$. HRMS (ESI) $\mathrm{m} / \mathrm{z}$ Calcd for $\mathrm{C}_{21} \mathrm{H}_{16} \mathrm{NO}_{2}[\mathrm{M}+\mathrm{H}]^{+} 314.1181$, found 314.1177 .

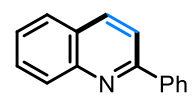

\section{2-Phenylquinoline (3p)}

White solid (22.6 mg, 55\% yield). PE/EA $=30: 1, \mathrm{R}_{\mathrm{f}}=0.27 . \mathrm{mp}: 87-89{ }^{\circ} \mathrm{C} .{ }^{1} \mathrm{H}$ NMR $(400$ $\left.\mathrm{MHz}, \mathrm{CDCl}_{3}\right): \delta 8.32-8.19(\mathrm{~m}, 4 \mathrm{H}), 7.94(\mathrm{~d}, J=8.6 \mathrm{~Hz}, 1 \mathrm{H}), 7.89(\mathrm{~d}, J=8.2 \mathrm{~Hz}, 1 \mathrm{H}), 7.79(\mathrm{t}, J=$ 
$7.8 \mathrm{~Hz}, 1 \mathrm{H}), 7.64-7.49(\mathrm{~m}, 4 \mathrm{H}) .{ }^{13} \mathrm{C}$ NMR $\left(100 \mathrm{MHz}, \mathrm{CDCl}_{3}\right): \delta 157.4,148.3,139.7,136.8,129.8$, 129.7, 129.3, 128.8, 127.6, 127.5, 127.2, 126.3, 119.0. IR (KBr): 3069, 1644, 1066, 827, 761, 683 $\mathrm{cm}^{-1}$. HRMS (ESI) m/z Calcd for $\mathrm{C}_{15} \mathrm{H}_{12} \mathrm{~N}[\mathrm{M}+\mathrm{H}]^{+}$206.0970, found 206.0968.

\section{6-Methyl-2-phenylquinoline (3q)}

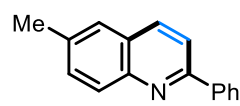

White solid (25.4 mg, 58\% yield). PE/EA $=30: 1, \mathrm{R}_{\mathrm{f}}=0.26 . \mathrm{mp}: 103-105{ }^{\circ} \mathrm{C} .{ }^{1} \mathrm{H}$ NMR $(400$ $\left.\mathrm{MHz}, \mathrm{CDCl}_{3}\right): \delta 8.23-8.15(\mathrm{~m}, 3 \mathrm{H}), 8.12(\mathrm{~d}, J=8.5 \mathrm{~Hz}, 1 \mathrm{H}), 7.88(\mathrm{~d}, J=8.6 \mathrm{~Hz}, 1 \mathrm{H}), 7.65-7.53$ (m, 4H), 7.53-7.46 (m, 1H), 2.60 (s, 3H). ${ }^{13} \mathrm{C}$ NMR (100 MHz, $\left.\mathrm{CDCl}_{3}\right): \delta 156.5,146.9,139.8$, 136.2, 136.1, 132.0, 129.4, 129.1, 128.8, 127.5, 127.2, 126.3, 119.0, 21.6. IR (KBr): 3011, 1642, 1561, 1189, 877, 862, $682 \mathrm{~cm}^{-1}$. HRMS (ESI) m/z Calcd for $\mathrm{C}_{16} \mathrm{H}_{14} \mathrm{~N}[\mathrm{M}+\mathrm{H}]^{+} 220.1126$, found 220.1126 .

6-(Methylthio)-2-phenylquinoline (3r)

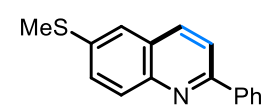

White solid (30.6 mg, 61\% yield). PE/EA $=30: 1, \mathrm{R}_{\mathrm{f}}=0.24 . \mathrm{mp}: 121-124{ }^{\circ} \mathrm{C} .{ }^{1} \mathrm{H}$ NMR $(400$ $\left.\mathrm{MHz}, \mathrm{CDCl}_{3}\right): \delta 8.21(\mathrm{~d}, J=7.4 \mathrm{~Hz}, 2 \mathrm{H}), 8.19-8.10(\mathrm{~m}, 2 \mathrm{H}), 7.92(\mathrm{~d}, J=8.6 \mathrm{~Hz}, 1 \mathrm{H}), 7.67(\mathrm{~d}, J=$ $8.9 \mathrm{~Hz}, 1 \mathrm{H}), 7.63-7.56(\mathrm{~m}, 3 \mathrm{H}), 7.55-7.49(\mathrm{~m}, 1 \mathrm{H}), 2.67$ (s, 3H). ${ }^{13} \mathrm{C} \mathrm{NMR}\left(100 \mathrm{MHz}, \mathrm{CDCl}_{3}\right): \delta$ 156.5, 146.5, 139.6, 137.1, 135.5, 129.9, 129.3, 129.2, 128.9, 127.7, 127.4, 122.3, 119.5, 15.7. IR (KBr): 1642, 1597, 1467, 1253, 1179, 823, 756, $684 \mathrm{~cm}^{-1}$. HRMS (ESI) m/z Calcd for $\mathrm{C}_{16} \mathrm{H}_{14} \mathrm{NS}$ $[\mathrm{M}+\mathrm{H}]^{+}$252.0847, found 252.0846.

\section{6-Fluoro-2-phenylquinoline (3s)}

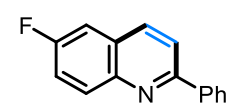

White solid $(19.2 \mathrm{mg}, 43 \%$ yield $) . \mathrm{PE} / \mathrm{EA}=30: 1, \mathrm{R}_{\mathrm{f}}=0.32 . \mathrm{mp}: 101-103{ }^{\circ} \mathrm{C} .{ }^{1} \mathrm{H}$ NMR $(400$ $\left.\mathrm{MHz}, \mathrm{CDCl}_{3}\right): \delta 8.26-8.14(\mathrm{~m}, 4 \mathrm{H}), 7.93(\mathrm{~d}, J=8.6 \mathrm{~Hz}, 1 \mathrm{H}), 7.62-7.43(\mathrm{~m}, 5 \mathrm{H}) .{ }^{13} \mathrm{C}$ NMR $(100$ $\left.\mathrm{MHz}, \mathrm{CDCl}_{3}\right): \delta 160.4(\mathrm{~d}, J=246.3 \mathrm{~Hz}), 156.8,145.4,139.4,136.1(\mathrm{~d}, J=5.2 \mathrm{~Hz}), 132.2,129.4$, $128.9,127.7(\mathrm{~d}, J=9.9 \mathrm{~Hz}), 127.4,119.8(\mathrm{~d}, J=25.7 \mathrm{~Hz}), 119.7,110.5(\mathrm{~d}, J=21.6 \mathrm{~Hz}) .{ }^{19} \mathrm{~F}$ NMR (376 MHz, $\mathrm{CDCl}_{3}$ ): $\delta$-113.70. IR (KBr): 1721, 1643, 1507, 1067, 832, 764, $684 \mathrm{~cm}^{-1}$. HRMS (ESI) $\mathrm{m} / \mathrm{z}$ Calcd for $\mathrm{C}_{15} \mathrm{H}_{11} \mathrm{FN}[\mathrm{M}+\mathrm{H}]^{+}$224.0876, found 224.0874.

\section{6-Phenyl-[1,3]dioxolo[4,5-g]quinoline (3t)}

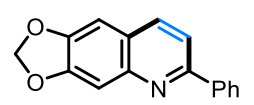

White solid (32.4 mg, 65\% yield). PE/EA $=30: 1, \mathrm{R}_{\mathrm{f}}=0.25 . \mathrm{mp}: 106-108{ }^{\circ} \mathrm{C} .{ }^{1} \mathrm{H}$ NMR $(400$ $\left.\mathrm{MHz}, \mathrm{CDCl}_{3}\right): \delta 8.16(\mathrm{~d}, J=7.6 \mathrm{~Hz}, 2 \mathrm{H}), 8.03(\mathrm{~d}, J=8.5 \mathrm{~Hz}, 1 \mathrm{H}), 7.75(\mathrm{~d}, J=8.5 \mathrm{~Hz}, 1 \mathrm{H})$, 7.58-7.42 (m, 4H), 7.09 (s, 1H), $\left.6.13(\mathrm{~s}, 2 \mathrm{H}) .{ }^{13} \mathrm{C} \mathrm{NMR} \mathrm{(100} \mathrm{MHz,} \mathrm{CDCl}_{3}\right): \delta 155.3,150.8,147.7$, 146.6, 139.8, 135.4, 128.8, 128.7, 127.2, 124.1, 117.1, 106.3, 102.5, 101.6. IR (KBr): 1644, 1462, 1229, 1040, 917, 858, 745, $687 \mathrm{~cm}^{-1}$. HRMS (ESI) m/z Calcd for $\mathrm{C}_{16} \mathrm{H}_{12} \mathrm{NO}_{2}[\mathrm{M}+\mathrm{H}]^{+} 250.0868$, found 250.0866 . 


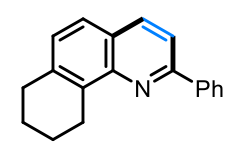

\section{2-Phenyl-7,8,9,10-tetrahydrobenzo $[h]$ quinoline (3u)}

White solid (34.7 mg, 67\% yield). PE/EA $=30: 1, \mathrm{R}_{\mathrm{f}}=0.28 . \mathrm{mp}: 118-120{ }^{\circ} \mathrm{C} .{ }^{1} \mathrm{H}$ NMR $(400$ $\left.\mathrm{MHz}, \mathrm{CDCl}_{3}\right): \delta 8.31(\mathrm{~d}, J=7.5 \mathrm{~Hz}, 2 \mathrm{H}), 8.18(\mathrm{~d}, J=8.6 \mathrm{~Hz}, 1 \mathrm{H}), 7.90(\mathrm{~d}, J=8.5 \mathrm{~Hz}, 1 \mathrm{H})$, 7.65-7.46 (m, 4H), $7.29(\mathrm{~d}, J=8.8 \mathrm{~Hz}, 1 \mathrm{H}), 3.58-3.48(\mathrm{~m}, 2 \mathrm{H}), 3.09-2.92(\mathrm{~m}, 2 \mathrm{H}), 2.10-1.92(\mathrm{~m}$, 4H). ${ }^{13} \mathrm{C}$ NMR (100 MHz, $\left.\mathrm{CDCl}_{3}\right): \delta 155.3,147.0,140.1,138.3,136.7,135.3,129.1,128.7,128.6$, 127.4, 125.3, 124.3, 117.4, 30.4, 24.8, 23.2, 23.0. IR (KBr): 3029, 1643, 1476, 1257, 837, 759, $684 \mathrm{~cm}^{-1}$. HRMS (ESI) $\mathrm{m} / \mathrm{z}$ Calcd for $\mathrm{C}_{19} \mathrm{H}_{18} \mathrm{~N}[\mathrm{M}+\mathrm{H}]^{+} 260.1439$, found 260.1437 .

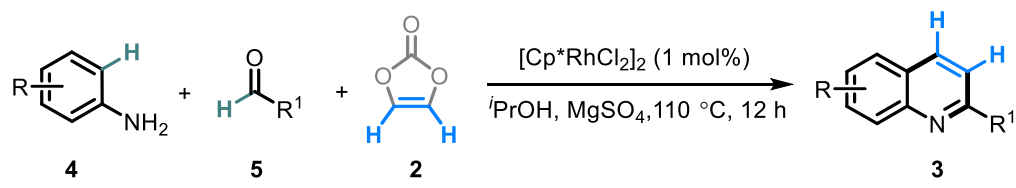

A pressure tube was charged with amines $4(0.3 \mathrm{mmol})$, aldehydes $5(0.3 \mathrm{mmol})$, anhydrous $\mathrm{MgSO}_{4}(0.6 \mathrm{mmol})$ vinylene carbonate $2(0.2 \mathrm{mmol}),\left[\mathrm{Cp} * \mathrm{RhCl}_{2}\right]_{2}(1.2 \mathrm{mg}, 0.002 \mathrm{mmol})$, and ${ }^{i} \mathrm{PrOH}(2.5 \mathrm{~mL})$. The reaction mixture was stirred at $110{ }^{\circ} \mathrm{C}$ for $12 \mathrm{~h}$ under $\mathrm{Ar}$ atmosphere in an oil bath. After cooling to room temperature, the mixture was filtered through a short Celite pad and all volatiles were removed under reduced pressure. The residue was purified by silica gel chromatography using PE/EA eluent to afford the product 3 .

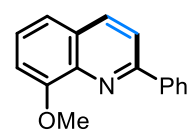

\section{8-Methoxy-2-phenylquinoline (3a')}

Colorless oil (24.5 mg, 52\% yield). PE/EA $=30: 1, \mathrm{R}_{\mathrm{f}}=0.23 .{ }^{1} \mathrm{H}$ NMR $\left(400 \mathrm{MHz}, \mathrm{CDCl}_{3}\right): \delta$ 8.32-8.19 (m, 3H), 7.97 (d, $J=8.6 \mathrm{~Hz}, 1 \mathrm{H}), 7.62-7.43(\mathrm{~m}, 5 \mathrm{H}), 7.14(\mathrm{~d}, J=7.4 \mathrm{~Hz}, 1 \mathrm{H}), 4.17$ (s, $3 \mathrm{H}) .{ }^{13} \mathrm{C} \mathrm{NMR}\left(100 \mathrm{MHz}, \mathrm{CDCl}_{3}\right): \delta 156.3,155.6,140.2,139.8,136.8,129.2,128.8,128.3,127.7$, 126.5, 119.5, 119.4, 108.1, 56.2. IR (KBr): 3011, 1641, 1526, 1378, 1107, 879, 736, $628 \mathrm{~cm}^{-1}$. HRMS (ESI) m/z Calcd for $\mathrm{C}_{16} \mathrm{H}_{14} \mathrm{NO}[\mathrm{M}+\mathrm{H}]^{+} 236.1075$, found 236.1072 .

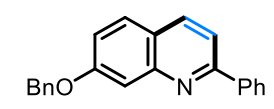

7-(Benzyloxy)-2-phenylquinoline (3b')

White solid (37.9 mg, $61 \%$ yield). PE/EA $=30: 1, \mathrm{R}_{\mathrm{f}}=0.24 . \mathrm{mp}: 93-95{ }^{\circ} \mathrm{C} .{ }^{1} \mathrm{H}$ NMR $(400$ $\left.\mathrm{MHz}, \mathrm{CDCl}_{3}\right): \delta 8.25-8.16(\mathrm{~m}, 3 \mathrm{H}), 7.84-7.75(\mathrm{~m}, 2 \mathrm{H}), 7.66(\mathrm{~s}, 1 \mathrm{H}), 7.63-7.38(\mathrm{~m}, 8 \mathrm{H})$, 7.37-7.33 (m, 1H), $5.31(\mathrm{~s}, 2 \mathrm{H}) .{ }^{13} \mathrm{C}$ NMR (100 MHz, $\left.\mathrm{CDCl}_{3}\right): \delta 160.0,157.8,149.9,139.9,136.5$, 136.4, 129.2, 128.8, 128.7, 128.6, 128.2, 127.7, 127.6, 127.6, 122.6, 119.9, 117.1, 108.8, 70.2. IR (KBr): 3033, 2890, 1616, 1451, 1380, 1187, 841, $687 \mathrm{~cm}^{-1}$. HRMS (ESI) m/z Calcd for $\mathrm{C}_{22} \mathrm{H}_{18} \mathrm{NO}$ $[\mathrm{M}+\mathrm{H}]^{+}$312.1388, found 312.1388.

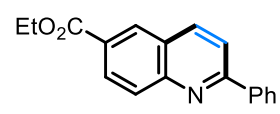

Ethyl 2-phenylquinoline-6-carboxylate (3c') 
White solid (33.2 mg, 60\% yield). PE/EA $=30: 1, \mathrm{R}_{\mathrm{f}}=0.28 . \mathrm{mp}: 95-97{ }^{\circ} \mathrm{C} .{ }^{1} \mathrm{H}$ NMR $(400$ $\left.\mathrm{MHz}, \mathrm{CDCl}_{3}\right): \delta 8.64(\mathrm{~s}, 1 \mathrm{H}), 8.36(\mathrm{~d}, J=8.7 \mathrm{~Hz}, 2 \mathrm{H}), 8.23(\mathrm{~d}, J=8.0 \mathrm{~Hz}, 3 \mathrm{H}), 7.99(\mathrm{~d}, J=8.6$ $\mathrm{Hz}, 1 \mathrm{H}), 7.68-7.47(\mathrm{~m}, 3 \mathrm{H}), 4.50(\mathrm{q}, J=7.1 \mathrm{~Hz}, 2 \mathrm{H}), 1.50(\mathrm{t}, J=7.2 \mathrm{~Hz}, 3 \mathrm{H}) .{ }^{13} \mathrm{C}$ NMR $(100$ $\left.\mathrm{MHz}, \mathrm{CDCl}_{3}\right): \delta 166.3,159.3,150.2,139.1,138.0,130.6,129.9,129.2,129.0,128.0,127.7,126.3$, 119.7, 61.3, 14.4. IR (KBr): 2987, 1706, 1677, 1275, 1090, 766, $682 \mathrm{~cm}^{-1}$. HRMS (ESI) m/z Calcd for $\mathrm{C}_{18} \mathrm{H}_{16} \mathrm{NO}_{2}[\mathrm{M}+\mathrm{H}]^{+}$278.1181, found 278.1178.

\section{2-Phenyl-6-(trifluoromethyl)quinoline (3d')}

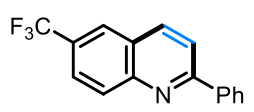

White solid (30.1 mg, 55\% yield). PE/EA $=30: 1, \mathrm{R}_{\mathrm{f}}=0.30 . \mathrm{mp}: 114-116^{\circ} \mathrm{C} .{ }^{1} \mathrm{H}$ NMR $(400$ $\left.\mathrm{MHz}, \mathrm{CDCl}_{3}\right): \delta 8.40-8.28(\mathrm{~m}, 2 \mathrm{H}), 8.27-8.16(\mathrm{~m}, 3 \mathrm{H}), 8.03(\mathrm{~d}, J=8.5 \mathrm{~Hz}, 1 \mathrm{H}), 7.94(\mathrm{~d}, J=8.9$ $\mathrm{Hz}, 1 \mathrm{H}), 7.64-7.51(\mathrm{~m}, 3 \mathrm{H}) .{ }^{13} \mathrm{C} \mathrm{NMR}\left(100 \mathrm{MHz}, \mathrm{CDCl}_{3}\right): \delta 159.4,149.3,139.0,137.5,130.9$, 130.0, 129.0, $128.0(\mathrm{q}, J=32.9 \mathrm{~Hz}), 127.7,126.1,125.5(\mathrm{q}, J=4.2 \mathrm{~Hz}), 125.4(\mathrm{q}, J=4.3 \mathrm{~Hz})$, $124.1(\mathrm{q}, J=275.0 \mathrm{~Hz}), 120.1 .{ }^{19} \mathrm{~F}$ NMR $\left(376 \mathrm{MHz}, \mathrm{CDCl}_{3}\right): \delta-62.18 . \mathrm{IR}(\mathrm{KBr}): 3021,1642$, 1567, 1117, 1069, 837, 757, $683 \mathrm{~cm}^{-1}$. HRMS (ESI) m/z Calcd for $\mathrm{C}_{16} \mathrm{H}_{11} \mathrm{~F}_{3} \mathrm{~N}[\mathrm{M}+\mathrm{H}]^{+} 274.0844$, found 274.0840 .

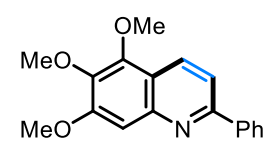

\section{5,6,7-Trimethoxy-2-phenylquinoline (3e')}

Colorless oil (40.2 mg, 68\% yield). PE/EA $=20: 1, \mathrm{R}_{\mathrm{f}}=0.21 .{ }^{1} \mathrm{H}$ NMR $\left(400 \mathrm{MHz}, \mathrm{CDCl}_{3}\right): \delta$ $8.47(\mathrm{~d}, J=8.7 \mathrm{~Hz}, 1 \mathrm{H}), 8.17(\mathrm{~d}, J=7.6 \mathrm{~Hz}, 2 \mathrm{H}), 7.79(\mathrm{~d}, J=8.6 \mathrm{~Hz}, 1 \mathrm{H}), 7.62-7.47(\mathrm{~m}, 3 \mathrm{H})$, 7.40 (s, 1H), 4.15 (s, 3H), 4.10 (s, 3H), 4.06 (s, 3H). ${ }^{13} \mathrm{C} \mathrm{NMR} \mathrm{(100} \mathrm{MHz,} \mathrm{CDCl}_{3}$ ): $\delta$ 157.0, 156.2, 147.0, 146.2, 140.8, 140.0, 131.1, 129.1, 128.8, 127.5, 118.2, 116.8, 104.7, 61.6, 61.3, 56.2. IR (KBr): 3011, 2901, 1606, 1473, 1387, 1241, 1102, 834, $688 \mathrm{~cm}^{-1}$. HRMS (ESI) m/z Calcd for $\mathrm{C}_{18} \mathrm{H}_{18} \mathrm{NO}_{3}[\mathrm{M}+\mathrm{H}]^{+} 296.1287$, found 296.1289 .

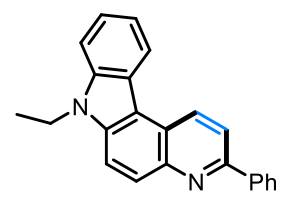

\section{7-Ethyl-3-phenyl-7H-pyrido[2,3-c]carbazole (3f')}

Yellow solid (45.1 mg, 70\% yield). PE/EA $=10: 1, R_{\mathrm{f}}=0.19 . \mathrm{mp}: 151-153{ }^{\circ} \mathrm{C} .{ }^{1} \mathrm{H}$ NMR $(400$ $\left.\mathrm{MHz}, \mathrm{CDCl}_{3}\right): \delta 9.14(\mathrm{~d}, J=8.7 \mathrm{~Hz}, 1 \mathrm{H}), 8.56(\mathrm{~d}, J=7.9 \mathrm{~Hz}, 1 \mathrm{H}), 8.32-8.23(\mathrm{~m}, 3 \mathrm{H}), 8.08(\mathrm{~d}, J=$ $8.7 \mathrm{~Hz}, 1 \mathrm{H}), 7.91(\mathrm{~d}, J=9.2 \mathrm{~Hz}, 1 \mathrm{H}), 7.66-7.54(\mathrm{~m}, 4 \mathrm{H}), 7.52(\mathrm{t}, J=7.0 \mathrm{~Hz}, 1 \mathrm{H}), 7.44(\mathrm{t}, J=8.0$ $\mathrm{Hz}, 1 \mathrm{H}), 4.54$ (q, $J=7.3 \mathrm{~Hz}, 2 \mathrm{H}), 1.54(\mathrm{t}, J=7.2 \mathrm{~Hz}, 3 \mathrm{H}) .{ }^{13} \mathrm{C} \mathrm{NMR}\left(100 \mathrm{MHz}, \mathrm{CDCl}_{3}\right): \delta 153.7$, 144.7, 140.0, 139.2, 137.0, 131.7, 128.8, 128.8, 128.6, 127.3, 124.5, 123.9, 123.4, 121.8, 120.0, 119.0, 114.4, 114.0, 109.5, 37.8, 14.4. IR (KBr): 2993, 1598, 1460, 1369, 1149, 1026, 823, 693 $\mathrm{cm}^{-1}$. HRMS (ESI) $\mathrm{m} / \mathrm{z}$ Calcd for $\mathrm{C}_{23} \mathrm{H}_{19} \mathrm{~N}_{2}[\mathrm{M}+\mathrm{H}]^{+} 323.1548$, found 323.1551 . 


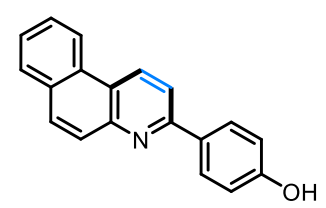

\section{4-(Benzo[f]quinolin-3-yl)phenol (3g')}

White solid (39.1 mg, 72\% yield). PE/EA $=3: 1, \mathrm{R}_{\mathrm{f}}=0.20 . \mathrm{mp}: 290-292{ }^{\circ} \mathrm{C} .{ }^{1} \mathrm{H}$ NMR $(400$ MHz, DMSO): $\delta 9.90(\mathrm{~s}, 1 \mathrm{H}), 9.23(\mathrm{~d}, J=8.8 \mathrm{~Hz}, 1 \mathrm{H}), 8.86(\mathrm{~d}, J=8.1 \mathrm{~Hz}, 1 \mathrm{H}), 8.27-8.16(\mathrm{~m}$, $3 \mathrm{H}), 8.13(\mathrm{~d}, J=9.1 \mathrm{~Hz}, 1 \mathrm{H}), 8.07(\mathrm{~d}, J=7.9 \mathrm{~Hz}, 1 \mathrm{H}), 7.97(\mathrm{~d}, J=9.1 \mathrm{~Hz}, 1 \mathrm{H}), 7.81-7.67(\mathrm{~m}$, 2H), $6.98(\mathrm{~d}, J=8.4 \mathrm{~Hz}, 2 \mathrm{H}) .{ }^{13} \mathrm{C}$ NMR (100 MHz, DMSO): $\delta 159.5,156.2,147.8,132.6,131.4$, 131.3, 129.8, 129.0, 128.5, 127.8, 127.6, 123.6, 123.6, 118.4, 116.1. IR (KBr): 3404, 2987, 1599 , 1467, 1253, 1179, 1026, 824, $748 \mathrm{~cm}^{-1}$. HRMS (ESI) m/z Calcd for $\mathrm{C}_{19} \mathrm{H}_{14} \mathrm{NO}[\mathrm{M}+\mathrm{H}]^{+} 272.1075$, found 272.1074 .

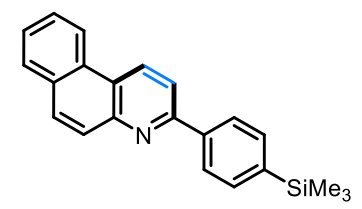

\section{3-(4-(Trimethylsilyl)phenyl)benzo[f $f$ quinoline $\left(3 h^{\prime}\right)$}

White solid (45.9 mg, 70\% yield). PE/EA $=20: 1, \mathrm{R}_{\mathrm{f}}=0.27 . \mathrm{mp}: 139-141{ }^{\circ} \mathrm{C} .{ }^{1} \mathrm{H}$ NMR $(400$ $\left.\mathrm{MHz}, \mathrm{CDCl}_{3}\right): \delta 9.03(\mathrm{~d}, J=8.6 \mathrm{~Hz}, 1 \mathrm{H}), 8.68(\mathrm{~d}, J=8.0 \mathrm{~Hz}, 1 \mathrm{H}), 8.26(\mathrm{~d}, J=7.6 \mathrm{~Hz}, 2 \mathrm{H}), 8.16$ $(\mathrm{d}, J=9.2 \mathrm{~Hz}, 1 \mathrm{H}), 8.07(\mathrm{~d}, J=8.8 \mathrm{~Hz}, 2 \mathrm{H}), 8.01(\mathrm{~d}, J=7.9 \mathrm{~Hz}, 1 \mathrm{H}), 7.83-7.67(\mathrm{~m}, 4 \mathrm{H}), 0.42(\mathrm{~s}$, 9H). ${ }^{13} \mathrm{C}$ NMR $\left(100 \mathrm{MHz}, \mathrm{CDCl}_{3}\right): \delta 156.9,148.3,141.8,139.8,133.9,131.7,131.5,131.0,129.7$ 128.7, 128.7, 127.1, 127.1, 126.6, 124.2, 122.7, 118.9, -1.1. IR (KBr): 3011, 1643, 1453, 1248, 1167, 1026, 827, $750 \mathrm{~cm}^{-1}$. HRMS (ESI) $\mathrm{m} / \mathrm{z}$ Calcd for $\mathrm{C}_{22} \mathrm{H}_{22} \mathrm{NSi}[\mathrm{M}+\mathrm{H}]^{+} 328.1522$, found 328.1517 .

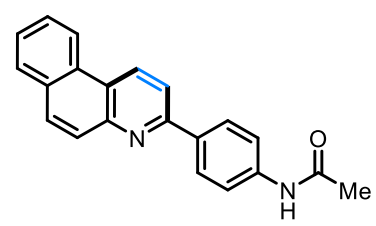

\section{N-(4-(benzo[f]quinolin-3-yl)phenyl)acetamide (3i')}

White solid (35.0 mg, 56\% yield). PE/EA $=2: 1, \mathrm{R}_{\mathrm{f}}=0.27 . \mathrm{mp}: 236-238{ }^{\circ} \mathrm{C} .{ }^{1} \mathrm{H}$ NMR $(400$ MHz, DMSO): $\delta 10.21(\mathrm{~s}, 1 \mathrm{H}), 9.28(\mathrm{~d}, J=8.8 \mathrm{~Hz}, 1 \mathrm{H}), 8.88(\mathrm{~d}, J=8.1 \mathrm{~Hz}, 1 \mathrm{H}), 8.32(\mathrm{~d}, J=8.4$ $\mathrm{Hz}, 2 \mathrm{H}), 8.27(\mathrm{~d}, J=8.7 \mathrm{~Hz}, 1 \mathrm{H}), 8.15(\mathrm{~d}, J=9.1 \mathrm{~Hz}, 1 \mathrm{H}), 8.09(\mathrm{~d}, J=7.8 \mathrm{~Hz}, 1 \mathrm{H}), 8.00(\mathrm{~d}, J=$ $9.1 \mathrm{~Hz}, 1 \mathrm{H}), 7.87-7.70$ (m, 4H), 2.14 (s, 3H). ${ }^{13} \mathrm{C}$ NMR (100 MHz, DMSO): $\delta$ 169.0, 155.8, 147.8, $141.2,133.4,132.8,131.5,131.5,129.8,129.1,128.5,128.1,127.9,127.7,124.0,123.7,119.5$, 118.7, 24.6. IR (KBr): 3010, 1658, 1492, 1253, 1156, 997, 835, $744 \mathrm{~cm}^{-1}$. HRMS (ESI) m/z Calcd for $\mathrm{C}_{21} \mathrm{H}_{17} \mathrm{~N}_{2} \mathrm{O}[\mathrm{M}+\mathrm{H}]^{+}$313.1341, found 313.1339.

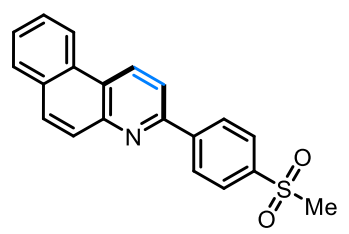


White solid (40.0 mg, 60\% yield). PE/EA $=5: 1, \mathrm{R}_{\mathrm{f}}=0.25 . \mathrm{mp}: 188-190{ }^{\circ} \mathrm{C} .{ }^{1} \mathrm{H}$ NMR $(400$ MHz, DMSO): $\delta 9.43(\mathrm{~d}, J=8.7 \mathrm{~Hz}, 1 \mathrm{H}), 8.96(\mathrm{~d}, J=8.0 \mathrm{~Hz}, 1 \mathrm{H}), 8.62(\mathrm{~d}, J=8.1 \mathrm{~Hz}, 2 \mathrm{H}), 8.44$ $(\mathrm{d}, J=8.7 \mathrm{~Hz}, 1 \mathrm{H}), 8.22(\mathrm{~d}, J=9.1 \mathrm{~Hz}, 1 \mathrm{H}), 8.19-8.10(\mathrm{~m}, 3 \mathrm{H}), 8.06(\mathrm{~d}, J=9.1 \mathrm{~Hz}, 1 \mathrm{H})$, 7.87-7.73 (m, 2H), 3.33 (s, 4H). ${ }^{13} \mathrm{C}$ NMR (100 MHz, DMSO): $\delta 154.3,147.9,143.6,141.8$, 133.3, 132.0, 131.9, 129.6, 129.1, 128.4, 128.3, 128.1, 128.0, 125.1, 124.0, 119.8, 44.1. IR (KBr): 3087, 1643, 1488, 1267, 1149, 928, 755, $676 \mathrm{~cm}^{-1}$. HRMS (ESI) m/z Calcd for $\mathrm{C}_{20} \mathrm{H}_{16} \mathrm{NO}_{2} \mathrm{~S}$ $[\mathrm{M}+\mathrm{H}]^{+} 334.0902$, found 334.0901 .

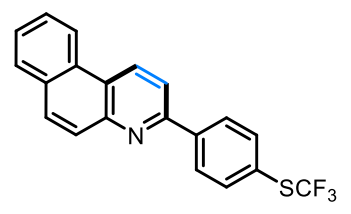

\section{3-(4-((Trifluoromethyl)thio)phenyl)benzo[f $f$ quinoline (31')}

White solid (44.1 mg, 62\% yield). PE/EA $=30: 1, \mathrm{R}_{\mathrm{f}}=0.26 . \mathrm{mp}: 185-187{ }^{\circ} \mathrm{C} .{ }^{1} \mathrm{H}$ NMR (400 $\left.\mathrm{MHz}, \mathrm{CDCl}_{3}\right): \delta 9.05(\mathrm{~d}, J=8.6 \mathrm{~Hz}, 1 \mathrm{H}), 8.67(\mathrm{~d}, J=8.0 \mathrm{~Hz}, 1 \mathrm{H}), 8.31(\mathrm{~d}, J=8.5 \mathrm{~Hz}, 2 \mathrm{H})$, 8.15-8.02 (m, 3H), $8.00(\mathrm{~d}, J=7.7 \mathrm{~Hz}, 1 \mathrm{H}), 7.86(\mathrm{~d}, J=8.1 \mathrm{~Hz}, 2 \mathrm{H}), 7.80-7.67(\mathrm{~m}, 2 \mathrm{H}) .{ }^{13} \mathrm{C}$ NMR $\left(100 \mathrm{MHz}, \mathrm{CDCl}_{3}\right): \delta 155.2,148.3,141.8,136.6,131.8,131.7,131.4,129.6$ (q, $J=279.4$ $\mathrm{Hz}), 129.5,128.8,128.5,128.3,127.5,127.3,125.3,124.6,122.7,118.7 .{ }^{19} \mathrm{~F}$ NMR $(376 \mathrm{MHz}$, $\left.\mathrm{CDCl}_{3}\right): \delta$-42.40. IR (KBr): 1648, 1596, 1488, 1232, 1114, 1026, 822, $753 \mathrm{~cm}^{-1}$. HRMS (ESI) m/z Calcd for $\mathrm{C}_{20} \mathrm{H}_{13} \mathrm{~F}_{3} \mathrm{NS}[\mathrm{M}+\mathrm{H}]^{+}$356.0721, found 356.0720.

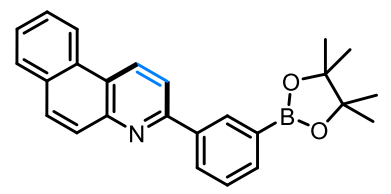

\section{3-(3-(4,4,5,5-Tetramethyl-1,3,2-dioxaborolan-2-yl)phenyl)benzo[f]quinoline (3m')}

White solid (48.1 mg, 63\% yield). PE/EA $=5: 1, \mathrm{R}_{\mathrm{f}}=0.22 . \mathrm{mp}: 155-157{ }^{\circ} \mathrm{C} .{ }^{1} \mathrm{H}$ NMR $(400$ $\left.\mathrm{MHz}, \mathrm{CDCl}_{3}\right): \delta 9.04(\mathrm{~d}, J=8.6 \mathrm{~Hz}, 1 \mathrm{H}), 8.69(\mathrm{~d}, J=8.1 \mathrm{~Hz}, 1 \mathrm{H}), 8.64(\mathrm{~s}, 1 \mathrm{H}), 8.42(\mathrm{~d}, J=7.8$ $\mathrm{Hz}, 1 \mathrm{H}), 8.14(\mathrm{~d}, J=8.8 \mathrm{~Hz}, 2 \mathrm{H}), 8.05(\mathrm{~d}, J=9.1 \mathrm{~Hz}, 1 \mathrm{H}), 7.98(\mathrm{t}, J=7.5 \mathrm{~Hz}, 2 \mathrm{H}), 7.80-7.66(\mathrm{~m}$, 2H), $7.61(\mathrm{t}, J=7.5 \mathrm{~Hz}, 1 \mathrm{H}), 1.44(\mathrm{~s}, 12 \mathrm{H}) .{ }^{13} \mathrm{C} \mathrm{NMR}\left(100 \mathrm{MHz}, \mathrm{CDCl}_{3}\right): \delta 156.9,148.2,138.8$, 135.7, 133.6, 131.7, 131.4, 130.9, 130.4, 129.7, 128.7, 128.4, 127.1, 127.0, 124.1, 122.7, 119.0, 84.0, 24.9. IR (KBr): 3016, 1642, 1360, 1249, 1144, 1046, 824, $695 \mathrm{~cm}^{-1}$. HRMS (ESI) m/z Calcd for $\mathrm{C}_{25} \mathrm{H}_{25} \mathrm{BNO}_{2}[\mathrm{M}+\mathrm{H}]^{+}$382.1978, found 382.1974.

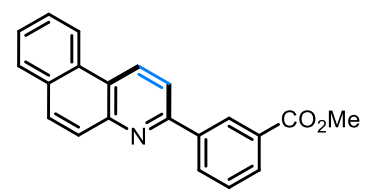

\section{Methyl 3-(benzo[f]quinolin-3-yl)benzoate (3n')}

White solid (35.1 mg, 56\% yield). PE/EA $=30: 1, \mathrm{R}_{\mathrm{f}}=0.23 . \mathrm{mp}: 120-122{ }^{\circ} \mathrm{C} .{ }^{1} \mathrm{H}$ NMR $(400$ MHz, $\left.\mathrm{CDCl}_{3}\right): \delta 9.06(\mathrm{~d}, J=8.7 \mathrm{~Hz}, 1 \mathrm{H}), 8.91(\mathrm{t}, J=1.8 \mathrm{~Hz}, 1 \mathrm{H}), 8.68(\mathrm{~d}, J=8.1 \mathrm{~Hz}, 1 \mathrm{H}), 8.52$ $(\mathrm{d}, J=7.8 \mathrm{~Hz}, 1 \mathrm{H}), 8.19(\mathrm{~d}, J=7.8 \mathrm{~Hz}, 1 \mathrm{H}), 8.16-8.04(\mathrm{~m}, 3 \mathrm{H}), 8.00(\mathrm{~d}, J=7.8 \mathrm{~Hz}, 1 \mathrm{H})$, 7.79-7.63 (m, 3H), 4.04 (s, 3H). ${ }^{13} \mathrm{C}$ NMR (100 MHz, $\left.\mathrm{CDCl}_{3}\right): \delta 167.0,155.5,148.2,139.7,131.8$, 131.7, 131.6, 131.2, 130.8, 130.2, 129.5, 129.0, 128.7, 128.5, 128.5, 127.3, 127.2, 124.4, 122.7, 118.6, 52.3. IR (KBr): 3064, 1720, 1640, 1246, 1076, 827, $753 \mathrm{~cm}^{-1}$. HRMS (ESI) m/z Calcd for 


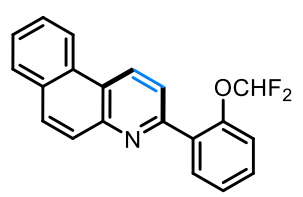

\section{3-(2-(Difluoromethoxy)phenyl)benzo[f $f$ quinoline $\left(3^{\prime}\right.$ ')}

White solid (39.2 mg, $61 \%$ yield). PE/EA $=30: 1, R_{\mathrm{f}}=0.27 . \mathrm{mp}: 201-203{ }^{\circ} \mathrm{C} .{ }^{1} \mathrm{H}$ NMR $(400$ $\mathrm{MHz}, \mathrm{CDCl}_{3}$ ): $\delta 9.03(\mathrm{~d}, J=8.7 \mathrm{~Hz}, 1 \mathrm{H}), 8.69(\mathrm{~d}, J=7.2 \mathrm{~Hz}, 1 \mathrm{H}), 8.16-7.93(\mathrm{~m}, 5 \mathrm{H}), 7.82-7.66$ $(\mathrm{m}, 2 \mathrm{H}), 7.58-7.42(\mathrm{~m}, 2 \mathrm{H}), 7.36(\mathrm{~d}, J=8.2 \mathrm{~Hz}, 1 \mathrm{H}), 6.64(\mathrm{t}, J=74.5 \mathrm{~Hz}, 1 \mathrm{H}) .{ }^{13} \mathrm{C}$ NMR $(100$ $\left.\mathrm{MHz}, \mathrm{CDCl}_{3}\right): \delta 154.8,149.1,148.2,133.1,132.0,131.8,131.1,130.9,130.3,129.6,128.8,128.4$ $127.4,127.2,126.3,124.3,122.8,122.8,120.7,116.8$ (t, $J=257.8 \mathrm{~Hz}) .{ }^{19} \mathrm{~F}$ NMR $(376 \mathrm{MHz}$, $\mathrm{CDCl}_{3}$ ): $\delta$-80.50. IR (KBr): 3026, 1621, 1467, 1210, 1124, 1039, 830, $753 \mathrm{~cm}^{-1} . \mathrm{HRMS}(\mathrm{ESI}) \mathrm{m} / \mathrm{z}$ Calcd for $\mathrm{C}_{20} \mathrm{H}_{14} \mathrm{~F}_{2} \mathrm{NO}[\mathrm{M}+\mathrm{H}]^{+} 322.1043$, found 322.1042 .

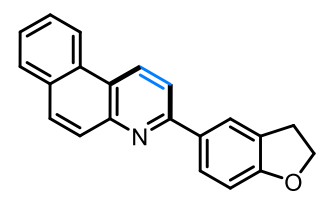

\section{3-(2,3-Dihydrobenzofuran-5-yl)benzo[f]quinoline (3p')}

White solid (32.1 mg, 54\% yield). PE/EA $=30: 1, \mathrm{R}_{\mathrm{f}}=0.24 . \mathrm{mp}: 179-181{ }^{\circ} \mathrm{C} .{ }^{1} \mathrm{H}$ NMR $(400$ $\left.\mathrm{MHz}, \mathrm{CDCl}_{3}\right): \delta 9.00(\mathrm{~d}, J=8.7 \mathrm{~Hz}, 1 \mathrm{H}), 8.67(\mathrm{~d}, J=8.2 \mathrm{~Hz}, 1 \mathrm{H}), 8.21(\mathrm{~s}, 1 \mathrm{H}), 8.14-7.95(\mathrm{~m}$, $5 \mathrm{H}), 7.80-7.64(\mathrm{~m}, 2 \mathrm{H}), 6.99(\mathrm{~d}, J=8.3 \mathrm{~Hz}, 1 \mathrm{H}), 4.73(\mathrm{t}, J=8.7 \mathrm{~Hz}, 2 \mathrm{H}), 3.39(\mathrm{t}, J=8.8 \mathrm{~Hz}, 2 \mathrm{H})$. ${ }^{13} \mathrm{C}$ NMR $\left(100 \mathrm{MHz}, \mathrm{CDCl}_{3}\right): \delta 161.5,156.9,148.2,132.3,131.5,131.4,130.9,129.7,128.7$, 128.6, 128.0, 127.8, 127.0, 126.9, 124.2, 123.6, 122.5, 118.4, 109.5, 71.8, 29.6. IR (KBr): 2987, $1639,1483,1238,1164,821,749,673 \mathrm{~cm}^{-1}$. HRMS (ESI) $\mathrm{m} / \mathrm{z}$ Calcd for $\mathrm{C}_{21} \mathrm{H}_{16} \mathrm{NO}[\mathrm{M}+\mathrm{H}]^{+}$ 298.1232, found 298.1231.

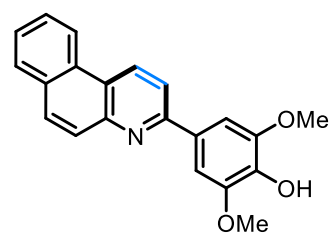

\section{4-(Benzo[f]quinolin-3-yl)-2,6-dimethoxyphenol (3q')}

White solid (49.1 mg, 74\% yield). PE/EA $=5: 1, \mathrm{R}_{\mathrm{f}}=0.32 . \mathrm{mp}: 138-140{ }^{\circ} \mathrm{C} .{ }^{1} \mathrm{H}$ NMR $(400$ $\left.\mathrm{MHz}, \mathrm{CDCl}_{3}\right): \delta 8.99(\mathrm{~d}, J=8.7 \mathrm{~Hz}, 1 \mathrm{H}), 8.65(\mathrm{~d}, J=8.1 \mathrm{~Hz}, 1 \mathrm{H}), 8.15-7.94(\mathrm{~m}, 4 \mathrm{H}), 7.79-7.63$ (m, 2H), 7.54 (s, 2H), 5.85 (s, 1H), 4.08 (s, 6H). $\left.{ }^{13} \mathrm{C} \mathrm{NMR} \mathrm{(100} \mathrm{MHz,} \mathrm{CDCl}_{3}\right): \delta$ 156.5, 148.1, 147.5, 136.4, 131.6, 131.5, 131.0, 130.9, 129.7, 128.7, 128.5, 127.1, 127.0, 123.9, 122.6, 118.4, 104.4, 56.5. IR (KBr): 3011, 1608, 1454, 1340, 1212, 1112, 826, $745 \mathrm{~cm}^{-1}$. HRMS (ESI) m/z Calcd for $\mathrm{C}_{21} \mathrm{H}_{18} \mathrm{NO}_{3}[\mathrm{M}+\mathrm{H}]^{+}$332.1287, found 332.1281.

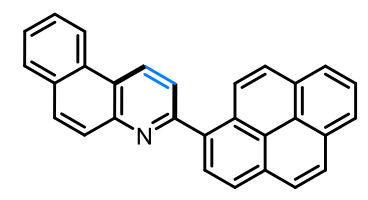

3-(Pyren-1-yl)benzo[f]quinoline (3r') 
White solid (50.1 mg, 67\% yield). PE/EA $=10: 1, \mathrm{R}_{\mathrm{f}}=0.27 . \mathrm{mp}: 216-218{ }^{\circ} \mathrm{C} .{ }^{1} \mathrm{H}$ NMR $(400$ $\left.\mathrm{MHz}, \mathrm{CDCl}_{3}\right): \delta 9.17(\mathrm{~d}, J=8.4 \mathrm{~Hz}, 1 \mathrm{H}), 8.77(\mathrm{~d}, J=8.1 \mathrm{~Hz}, 1 \mathrm{H}), 8.56(\mathrm{~d}, J=9.3 \mathrm{~Hz}, 1 \mathrm{H}), 8.38$ (s, 2H), 8.31-8.21 (m, 3H), 8.19 (s, 2H), 8.17-8.03 (m, 5H), 7.85-7.72 (m, 2H). ${ }^{13} \mathrm{C}$ NMR (100 $\left.\mathrm{MHz}, \mathrm{CDCl}_{3}\right): \delta 159.2,148.3,135.8,131.8,131.6,131.4,131.2,131.0,131.0,129.7,128.9,128.8$, 128.6, 128.2, 128.0, 127.9, 127.5, 127.3, 127.2, 126.1, 125.4, 125.1, 125.0, 124.9, 124.8, 123.9, 123.8, 122.7. IR (KBr): 3058, 1643, 1487, 1265, 834, $748 \mathrm{~cm}^{-1}$. HRMS (ESI) m/z Calcd for $\mathrm{C}_{29} \mathrm{H}_{18} \mathrm{~N}[\mathrm{M}+\mathrm{H}]^{+} 380.1439$, found 380.1434 .

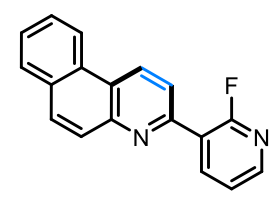

\section{3-(2-Fluoropyridin-3-yl)benzo[f]quinoline (3s')}

White solid (32.4 mg, 59\% yield). PE/EA $=10: 1, \mathrm{R}_{\mathrm{f}}=0.29 . \mathrm{mp}: 197-199{ }^{\circ} \mathrm{C} .{ }^{1} \mathrm{H}$ NMR $(400$ MHz, $\left.\mathrm{CDCl}_{3}\right): \delta 9.06(\mathrm{~d}, J=8.7 \mathrm{~Hz}, 1 \mathrm{H}), 8.83(\mathrm{t}, J=8.4 \mathrm{~Hz}, 1 \mathrm{H}), 8.69(\mathrm{~d}, J=8.0 \mathrm{~Hz}, 1 \mathrm{H}), 8.36$ $(\mathrm{d}, J=4.7 \mathrm{~Hz}, 1 \mathrm{H}), 8.20(\mathrm{~d}, J=8.7 \mathrm{~Hz}, 1 \mathrm{H}), 8.12-7.97(\mathrm{~m}, 3 \mathrm{H}), 7.80-7.69(\mathrm{~m}, 2 \mathrm{H}), 7.45(\mathrm{t}, J=$ $6.2 \mathrm{~Hz}, 1 \mathrm{H}) .{ }^{13} \mathrm{C}$ NMR $\left(100 \mathrm{MHz}, \mathrm{CDCl}_{3}\right): \delta 161.1(\mathrm{~d}, J=239.5 \mathrm{~Hz}), 151.0(\mathrm{~d}, J=6.5 \mathrm{~Hz}), 148.3$, 148.0, 147.8, 141.9, 131.9, 131.3, 129.4, 128.8, 128.2, 127.6, 127.3, 124.7, 122.9, 122.6, 122.2, $121.8(\mathrm{~d}, J=10.2 \mathrm{~Hz}) .{ }^{19} \mathrm{~F}$ NMR (376 MHz, $\left.\mathrm{CDCl}_{3}\right): \delta$-69.14. IR (KBr): 3086, 1643, 1567, 1412 , 1242, 1072, 818, $749 \mathrm{~cm}^{-1}$. HRMS (ESI) $\mathrm{m} / \mathrm{z}$ Calcd for $\mathrm{C}_{18} \mathrm{H}_{12} \mathrm{FN}_{2}[\mathrm{M}+\mathrm{H}]^{+} 275.0985$, found 275.0979 .

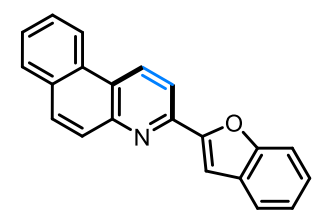

\section{3-(Benzofuran-2-yl)benzo[f]quinoline (3t')}

White solid (37.8 mg, $64 \%$ yield). PE/EA $=20: 1, R_{\mathrm{f}}=0.25 . \mathrm{mp}: 168-170{ }^{\circ} \mathrm{C} .{ }^{1} \mathrm{H}$ NMR $(400$ $\left.\mathrm{MHz}, \mathrm{CDCl}_{3}\right): \delta 9.09(\mathrm{~d}, J=8.7 \mathrm{~Hz}, 1 \mathrm{H}), 8.70(\mathrm{~d}, J=8.1 \mathrm{~Hz}, 1 \mathrm{H}), 8.24(\mathrm{~d}, J=8.7 \mathrm{~Hz}, 1 \mathrm{H})$, 8.18-8.06 (m, 2H), $8.02(\mathrm{~d}, J=7.9 \mathrm{~Hz}, 1 \mathrm{H}), 7.83-7.68(\mathrm{~m}, 5 \mathrm{H}), 7.44(\mathrm{t}, J=7.9 \mathrm{~Hz}, 1 \mathrm{H}), 7.37(\mathrm{~d}, J$ $=7.6 \mathrm{~Hz}, 1 \mathrm{H}) .{ }^{13} \mathrm{C}$ NMR $\left(100 \mathrm{MHz}, \mathrm{CDCl}_{3}\right): \delta 155.6,155.3,148.6,148.3,131.8,131.4,129.6$, $129.0,128.8,128.3,127.4,127.3,125.5,124.8,123.3,122.7,121.8,118.1,111.8,105.9 . \mathrm{IR}(\mathrm{KBr})$ 3012, 1637, 1488, 1269, 1183, 1068, 822, $742 \mathrm{~cm}^{-1}$. HRMS (ESI) m/z Calcd for $\mathrm{C}_{21} \mathrm{H}_{14} \mathrm{NO}$ $[\mathrm{M}+\mathrm{H}]^{+}$296.1075, found 296.1072 .

\section{Synthetic transformation of products}

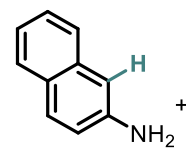

$4 a$

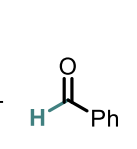

$5 a$

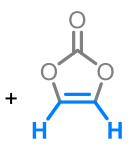

2

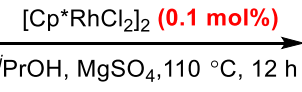

$5.0 \mathrm{mmo}$

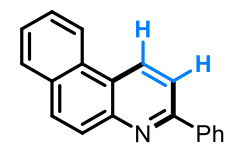

3a, $65 \%$

A pressure tube was charged with 2-naphthylamine $4 \mathbf{a}(1.0 \mathrm{~g}, 7.5 \mathrm{mmol})$, benzaldehyde $\mathbf{5 a}$ (0.8 g, $7.5 \mathrm{mmol})$, anhydrous $\mathrm{MgSO}_{4}(1.8 \mathrm{~g}, 15 \mathrm{mmol})$ vinylene carbonate 2 (430.2 mg, $\left.5.0 \mathrm{mmol}\right)$ $\left[\mathrm{Cp}^{*} \mathrm{RhCl}_{2}\right]_{2}(3.0 \mathrm{mg}, 0.005 \mathrm{mmol})$, and ${ }^{i} \mathrm{PrOH}(50 \mathrm{~mL})$. The reaction mixture was stirred at 
$110{ }^{\circ} \mathrm{C}$ for $12 \mathrm{~h}$ under Ar atmosphere in an oil bath. After cooling to room temperature, the mixture was filtered through a short Celite pad and all volatiles were removed under reduced pressure. The residue was purified by silica gel chromatography using PE/EA eluent to afford the product 3a $(829.1 \mathrm{mg}, 65 \%$ yield$)$.

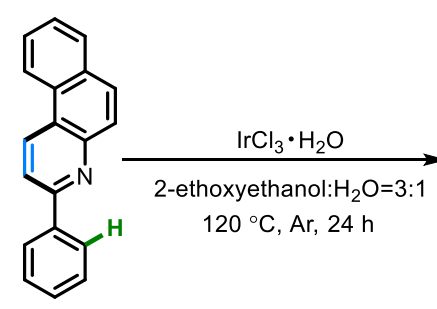

$3 a$

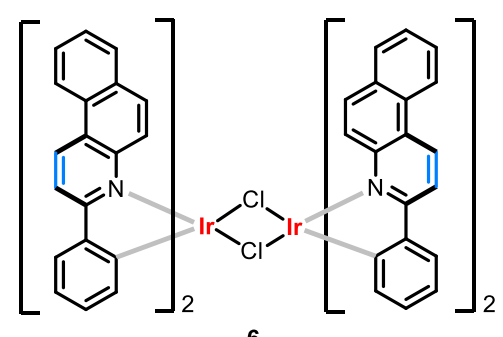

A pressure tube was charged with 3-phenylbenzo[f]quinoline 3a $(56.1 \mathrm{mg}, 0.22 \mathrm{mmol})$, $\mathrm{IrCl}_{3} \cdot \mathrm{H}_{2} \mathrm{O}(31.6 \mathrm{mg}, 0.1 \mathrm{mmol})$, 2-ethoxyethanol $(0.6 \mathrm{~mL})$ and $\mathrm{H}_{2} \mathrm{O}(0.2 \mathrm{~mL})$. The reaction mixture was stirred at $120^{\circ} \mathrm{C}$ for $24 \mathrm{~h}$ under Ar atmosphere in an oil bath. After cooling to room temperature, the precipitate was filtered off and washed with water. The solid was dissolved in dichloromethane and recrystallized in DCM/hexane to afford the product 6: Red solid (68.1 mg, 87\% yield). ${ }^{1} \mathrm{H}$ NMR (400 MHz, DMSO- $\left.d_{6}\right): \delta 9.58-9.46(\mathrm{~m}, 2 \mathrm{H}), 9.05(\mathrm{~d}, J=8.4 \mathrm{~Hz}, 1 \mathrm{H}), 8.54$ $(\mathrm{d}, J=8.8 \mathrm{~Hz}, 1 \mathrm{H}), 8.11-7.95(\mathrm{~m}, 3 \mathrm{H}), 7.89-7.73(\mathrm{~m}, 2 \mathrm{H}), 6.88(\mathrm{t}, J=7.5 \mathrm{~Hz}, 1 \mathrm{H}), 6.53(\mathrm{t}, J=$ $7.5 \mathrm{~Hz}, 1 \mathrm{H}), 6.25$ (d, $J=7.8 \mathrm{~Hz}, 1 \mathrm{H}) .{ }^{13} \mathrm{C}$ NMR (100 MHz, DMSO): $\delta$ 169.4, 149.7, 147.3, 145.5, 134.5, 134.4, 131.6, 131.3, 129.5, 129.0, 128.8, 128.5, 128.1, 128.0, 126.2, 124.8, 123.8, 121.4, 117.4 .<smiles>c1ccc(-c2ccc3ccccc3n2)cc1</smiles>

3a

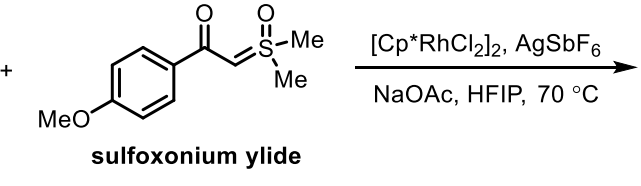

sulfoxonium ylide

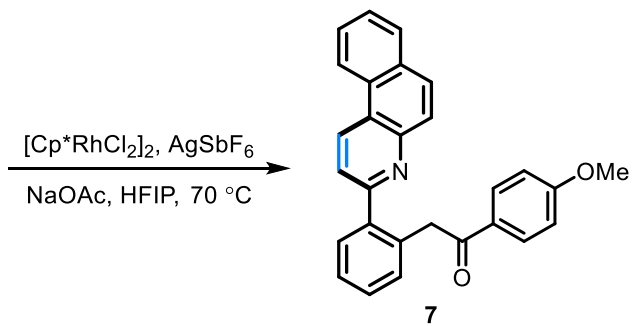

A pressure tube was charged with 3-phenylbenzo[f]quinoline 3a (51.0 $\mathrm{mg}, 0.2 \mathrm{mmol})$, 2-(dimethyl(oxo)- $\lambda^{6}$-sulfanylidene)-1-(4-methoxyphenyl)ethan-1-one $\quad(67.9 \mathrm{mg}, \quad 0.3 \mathrm{mmol})$, $\left[\mathrm{Cp}^{*} \mathrm{RhCl}_{2}\right]_{2}(6.0 \mathrm{mg}, 0.01 \mathrm{mmol}), \operatorname{AgSbF}_{6}(13.7 \mathrm{mg}, 0.04 \mathrm{mmol}), \mathrm{NaOAc}(16 \mathrm{mg}, 0.2 \mathrm{mmol})$ and HFIP $(1.6 \mathrm{~mL})$. The reaction mixture was stirred at $70{ }^{\circ} \mathrm{C}$ for $15 \mathrm{~h}$ under Air atmosphere in an oil bath. After cooling to room temperature, the mixture was filtered through a short Celite pad and all volatiles were removed under reduced pressure. The residue was purified by silica gel chromatography using PE/EA eluent to afford the product 7: White solid (63.4 mg, 79\% yield). $\mathrm{PE} / \mathrm{EA}=10: 1, \mathrm{R}_{\mathrm{f}}=0.25 . \mathrm{mp}: 136-138{ }^{\circ} \mathrm{C} .{ }^{1} \mathrm{H} \mathrm{NMR}\left(400 \mathrm{MHz}, \mathrm{CDCl}_{3}\right): \delta 8.98(\mathrm{~d}, J=8.6 \mathrm{~Hz}, 1 \mathrm{H})$ $8.63(\mathrm{~d}, J=8.1 \mathrm{~Hz}, 1 \mathrm{H}), 7.98-7.86(\mathrm{~m}, 4 \mathrm{H}), 7.79(\mathrm{~d}, J=8.7 \mathrm{~Hz}, 1 \mathrm{H}), 7.76-7.63(\mathrm{~m}, 4 \mathrm{H})$, $7.53-7.41(\mathrm{~m}, 3 \mathrm{H}), 6.84(\mathrm{~d}, J=8.3 \mathrm{~Hz}, 2 \mathrm{H}), 4.62(\mathrm{~s}, 2 \mathrm{H}), 3.81(\mathrm{~s}, 3 \mathrm{H}) .{ }^{13} \mathrm{C}$ NMR $(100 \mathrm{MHz}$, $\left.\mathrm{CDCl}_{3}\right): \delta 196.7,163.3,159.2,147.4,140.0,134.0,132.1,131.6,131.4,130.8,130.6,130.2,130.0$ $129.5,128.8,128.7,128.2,127.3,127.2,127.1,123.7,122.7,122.1,113.6,55.4,43.2$. IR (KBr): 3067, 1772, 1428, 1385, 1196, 752, $678 \mathrm{~cm}^{-1}$. HRMS (ESI) m/z Calcd for $\mathrm{C}_{28} \mathrm{H}_{22} \mathrm{NO}_{2}[\mathrm{M}+\mathrm{H}]^{+}$ 404.1651, found 404.1647. 


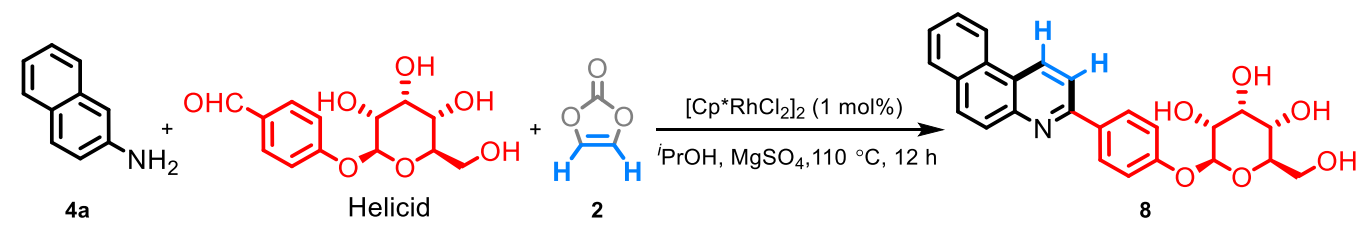

A pressure tube was charged with 2-naphthylamine $\mathbf{4 a}(42.9 \mathrm{mg}, 0.3 \mathrm{mmol})$, Helicid (85.3 $\mathrm{mg}, 0.3 \mathrm{mmol})$, anhydrous $\mathrm{MgSO}_{4}(72.0 \mathrm{mg}, 0.3 \mathrm{mmol})$ vinylene carbonate $2(17.2 \mathrm{mg}, 0.2 \mathrm{mmol})$, $\left[\mathrm{Cp}^{*} \mathrm{RhCl}_{2}\right]_{2}(1.2 \mathrm{mg}, 0.002 \mathrm{mmol})$, and ${ }^{i} \mathrm{PrOH}(2.5 \mathrm{~mL})$. The reaction mixture was stirred at $110{ }^{\circ} \mathrm{C}$ for $12 \mathrm{~h}$ under $\mathrm{Ar}$ atmosphere in an oil bath. After cooling to room temperature, the mixture was filtered through a short Celite pad and all volatiles were removed under reduced pressure. The residue was purified by silica gel chromatography using PE/EA eluent to afford the product 8: White solid (59.7 $\mathrm{mg}, 69 \%$ yield). $\mathrm{PE} / \mathrm{EA}=2: 1, \mathrm{R}_{\mathrm{f}}=0.32 . \mathrm{mp}: 240-243{ }^{\circ} \mathrm{C} .{ }^{1} \mathrm{H} \mathrm{NMR}$ (400 MHz, DMSO- $\left.d_{6}\right): \delta 9.30(\mathrm{~d}, J=8.8 \mathrm{~Hz}, 1 \mathrm{H}), 8.90(\mathrm{~d}, J=8.2 \mathrm{~Hz}, 1 \mathrm{H}), 8.37-8.25(\mathrm{~m}, 3 \mathrm{H})$, $8.16(\mathrm{~d}, J=9.1 \mathrm{~Hz}, 1 \mathrm{H}), 8.10(\mathrm{~d}, J=7.8 \mathrm{~Hz}, 1 \mathrm{H}), 8.00(\mathrm{~d}, J=9.1 \mathrm{~Hz}, 1 \mathrm{H}), 7.83-7.69(\mathrm{~m}, 2 \mathrm{H})$, $7.23(\mathrm{~d}, J=8.5 \mathrm{~Hz}, 2 \mathrm{H}), 5.28(\mathrm{~d}, J=7.8 \mathrm{~Hz}, 1 \mathrm{H}), 5.17(\mathrm{~d}, J=6.7 \mathrm{~Hz}, 1 \mathrm{H}), 5.02(\mathrm{~s}, 1 \mathrm{H}), 4.71(\mathrm{~d}, J$ $=7.4 \mathrm{~Hz}, 1 \mathrm{H}), 4.57(\mathrm{t}, J=5.7 \mathrm{~Hz}, 1 \mathrm{H}), 4.00(\mathrm{~s}, 1 \mathrm{H}), 3.82-3.72(\mathrm{~m}, 2 \mathrm{H}), 3.58-3.44(\mathrm{~m}, 3 \mathrm{H}) .{ }^{13} \mathrm{C}$ NMR (100 MHz, $\left.\mathrm{CDCl}_{3}\right): \delta 159.4,155.8,147.8,132.8,132.4,131.5,131.5,129.8,129.1,128.8$, $128.5,127.9,127.7,123.9,123.7,118.7,116.9,98.8,75.2,72.0,70.8$, 67.5, 61.4. IR (KBr): 3489, $3054,1716,1431,1239,1192,759,678 \mathrm{~cm}^{-1}$. HRMS (ESI) m/z Calcd for $\mathrm{C}_{25} \mathrm{H}_{24} \mathrm{NO}_{6}[\mathrm{M}+\mathrm{H}]^{+}$ 434.1604, found 434.1600.

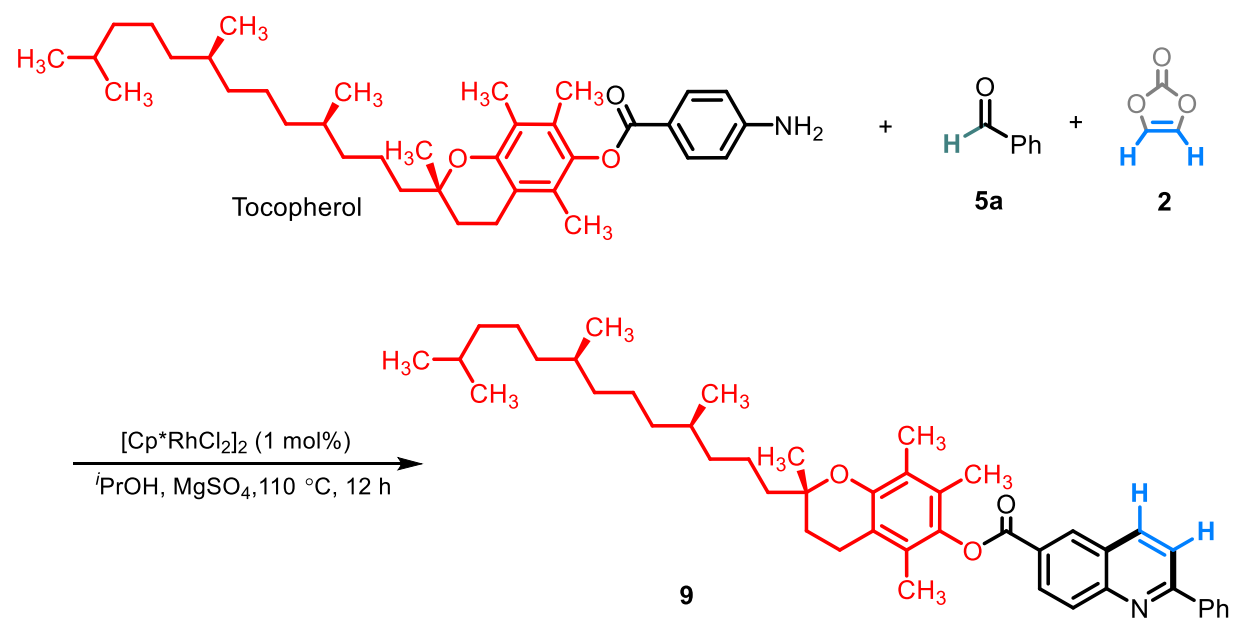

A pressure tube was charged with DL- $\alpha$-Tocopherol 4-amino benzoate (164.9 $\mathrm{mg}, 0.3 \mathrm{mmol}$ ), benzaldehyde $5 \mathbf{a}(31.8 \mathrm{mg}, 0.3 \mathrm{mmol})$, anhydrous $\mathrm{MgSO}_{4}(72.0 \mathrm{mg}, 0.3 \mathrm{mmol})$ vinylene carbonate $2(17.2 \mathrm{mg}, 0.2 \mathrm{mmol}),\left[\mathrm{Cp}^{*} \mathrm{RhCl}_{2}\right]_{2}(1.2 \mathrm{mg}, 0.002 \mathrm{mmol})$, and ${ }^{i} \mathrm{PrOH}(2.5 \mathrm{~mL})$. The reaction mixture was stirred at $110{ }^{\circ} \mathrm{C}$ for $12 \mathrm{~h}$ under Ar atmosphere in an oil bath. After cooling to room temperature, the mixture was filtered through a short Celite pad and all volatiles were removed under reduced pressure. The residue was purified by silica gel chromatography using PE/EA eluent to afford the product 9: White solid (54.2 $\mathrm{mg}, 41 \%$ yield). PE/EA $=30: 1, \mathrm{R}_{\mathrm{f}}=0.24 . \mathrm{mp}$ : 97-99 ${ }^{\circ} \mathrm{C} .{ }^{1} \mathrm{H}$ NMR (400 MHz, $\left.\mathrm{CDCl}_{3}\right): \delta 8.85(\mathrm{~s}, 1 \mathrm{H}), 8.53(\mathrm{~d}, J=9.0 \mathrm{~Hz}, 1 \mathrm{H}), 8.41(\mathrm{~d}, J=8.8$ $\mathrm{Hz}, 1 \mathrm{H}), 8.35-8.22(\mathrm{~m}, 3 \mathrm{H}), 8.02(\mathrm{~d}, J=8.6 \mathrm{~Hz}, 1 \mathrm{H}), 7.65-7.50(\mathrm{~m}, 3 \mathrm{H}), 2.73-2.63(\mathrm{~m}, 2 \mathrm{H}), 2.18$ $(\mathrm{s}, 3 \mathrm{H}), 2.14(\mathrm{~s}, 3 \mathrm{H}), 2.10(\mathrm{~s}, 3 \mathrm{H}), 1.93-1.76(\mathrm{~m}, 2 \mathrm{H}), 1.64-1.50(\mathrm{~m}, 4 \mathrm{H}), 1.35-1.24(\mathrm{~m}, 14 \mathrm{H})$, 1.16-1.09 (m, 3H), 0.95-0.85 (m, 15H). $\left.{ }^{13} \mathrm{C} \mathrm{NMR} \mathrm{(100} \mathrm{MHz,} \mathrm{CDCl}_{3}\right): \delta 164.9,159.6,150.5$, 
149.6, 140.7, 139.1, 138.1, 131.4, 130.2, 130.0, 129.6, 129.0, 127.8, 127.2, 126.9, 126.4, 125.2, $123.3,119.8,117.6,75.2,39.4,37.5,37.4,37.3,32.8,29.7,28.0,24.8,24.5,24.2,23.8,22.8,22.7$, 21.1, 20.7, 19.8, 19.7, 13.2, 12.3, 11.9. IR (KBr): 3017, 2981, 1730, 1461, 1239, 1090, 767, 683 $\mathrm{cm}^{-1}$. HRMS (ESI) $\mathrm{m} / \mathrm{z}$ Calcd for $\mathrm{C}_{45} \mathrm{H}_{60} \mathrm{NO}_{3}[\mathrm{M}+\mathrm{H}]^{+}$662.4573, found 662.4567 .

\section{E. Mechanistic study}

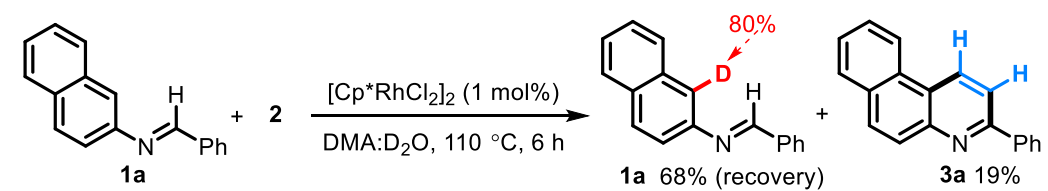

A pressure tube was charged with imine 1a $(0.30 \mathrm{mmol})$, vinylene carbonate $\mathbf{2}(0.20 \mathrm{mmol})$ $\left[\mathrm{Cp}^{*} \mathrm{RhCl}_{2}\right]_{2}(1.2 \mathrm{mg}, 0.002 \mathrm{mmol}), \mathrm{D}_{2} \mathrm{O}(0.1 \mathrm{~mL})$ and DMA $(1.0 \mathrm{~mL})$. The reaction mixture was stirred at $110{ }^{\circ} \mathrm{C}$ for $12 \mathrm{~h}$ under Ar atmosphere in an oil bath. After cooling to room temperature, all volatiles were removed under reduced pressure. The residue was purified by silica gel chromatography using PE/EA eluent to afford the substrate 1a and product 3a.
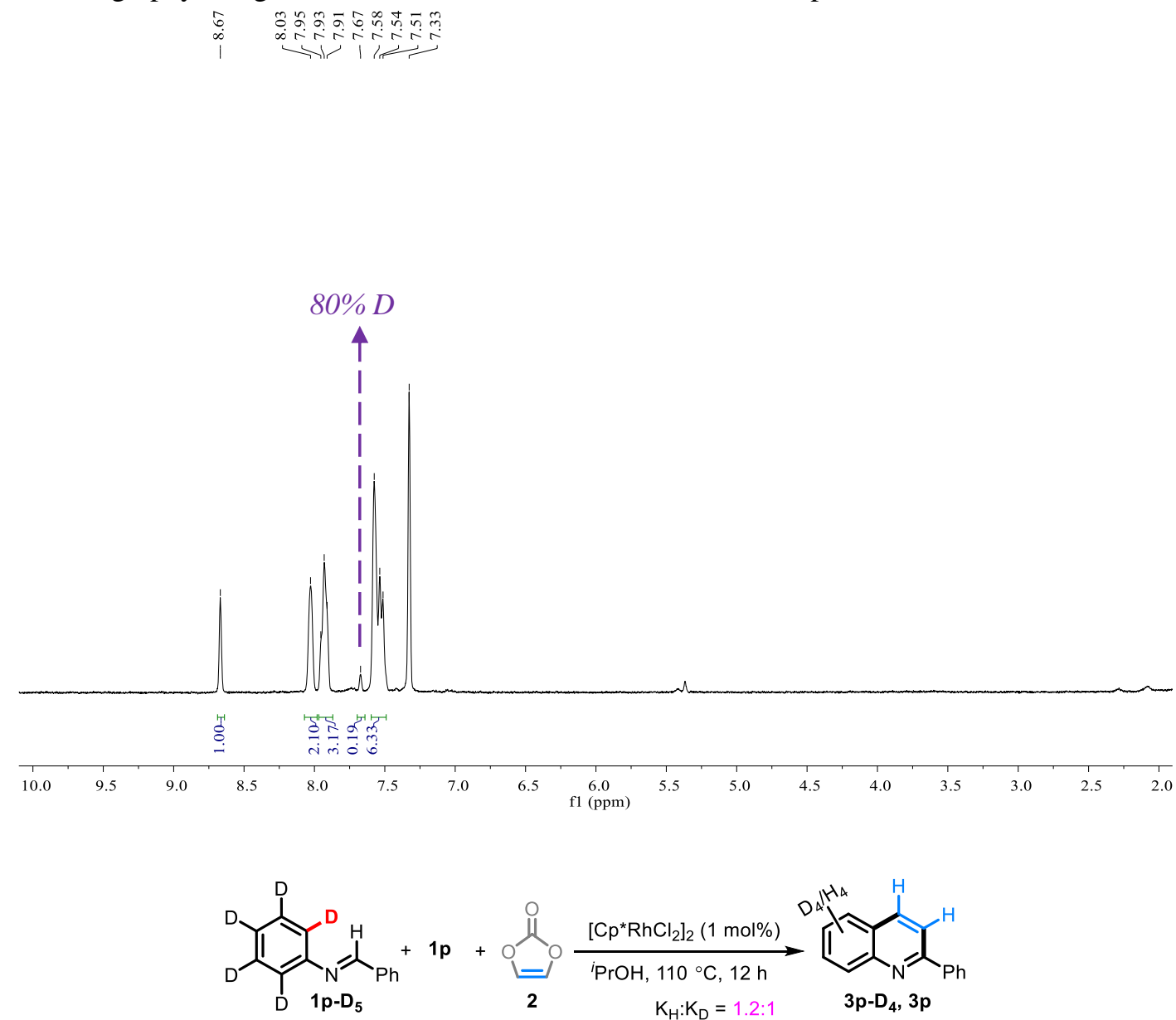

A pressure tube was charged with imine $1 \mathbf{p}(0.20 \mathrm{mmol}), \mathbf{1 p}-\mathbf{D}_{\mathbf{5}}(0.20 \mathrm{mmol})$, vinylene carbonate $2(0.20 \mathrm{mmol}),\left[\mathrm{Cp} * \mathrm{RhCl}_{2}\right]_{2}(1.2 \mathrm{mg}, 0.002 \mathrm{mmol})$ and ${ }^{i} \mathrm{PrOH}(2.0 \mathrm{~mL})$. The reaction mixture was stirred at $110{ }^{\circ} \mathrm{C}$ for $12 \mathrm{~h}$ under Ar atmosphere in an oil bath. After cooling to room temperature, all volatiles were removed under reduced pressure. The residue was purified by silica

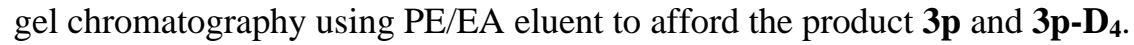




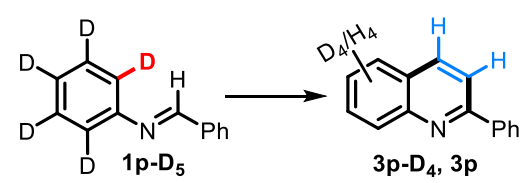

$\mathrm{n}(\mathrm{H})=0.55$

$\mathrm{n}(\mathrm{D})=0.45$

$\mathrm{KIE}=K_{\mathrm{H}} / K_{\mathrm{D}}=1.2$
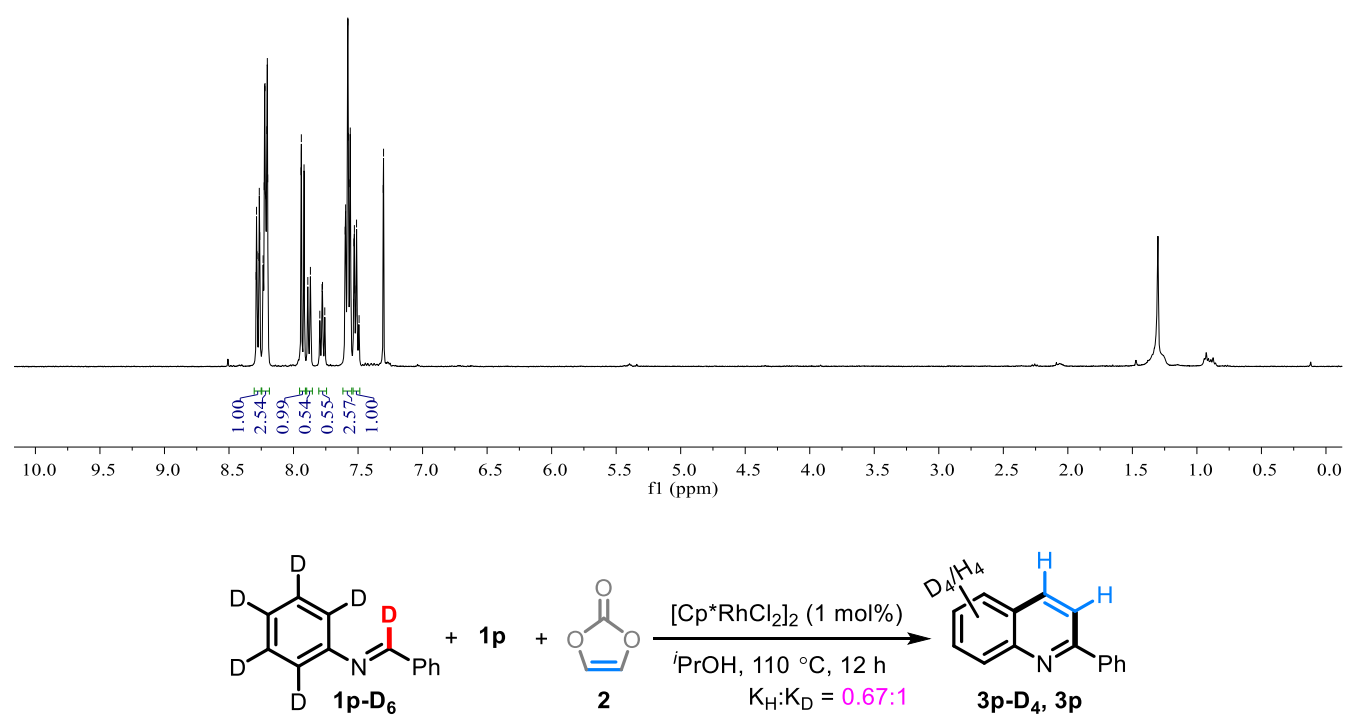

A pressure tube was charged with imine $1 \mathbf{p}(0.20 \mathrm{mmol}), \mathbf{1 p}-\mathbf{D}_{\mathbf{6}}(0.20 \mathrm{mmol})$, vinylene carbonate $2(0.20 \mathrm{mmol}),\left[\mathrm{Cp} * \mathrm{RhCl}_{2}\right]_{2}(1.2 \mathrm{mg}, 0.002 \mathrm{mmol})$ and ${ }^{i} \mathrm{PrOH}(2.0 \mathrm{~mL})$. The reaction mixture was stirred at $110{ }^{\circ} \mathrm{C}$ for $12 \mathrm{~h}$ under Ar atmosphere in an oil bath. After cooling to room temperature, all volatiles were removed under reduced pressure. The residue was purified by silica gel chromatography using PE/EA eluent to afford the product $\mathbf{3 p}$ and $\mathbf{3 p}-\mathbf{D}_{\mathbf{4}}$.

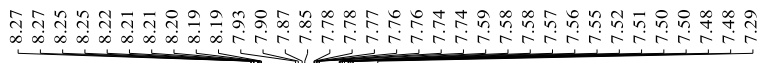

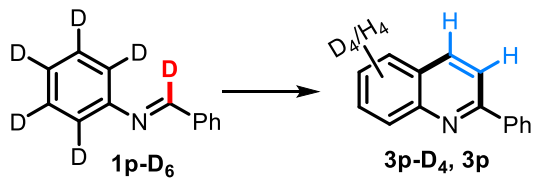

$\mathrm{n}(\mathrm{H})=0.40$

$\mathrm{n}(\mathrm{D})=0.60$

$\mathrm{KIE}=K_{\mathrm{H}} / K_{\mathrm{D}}=0.67$

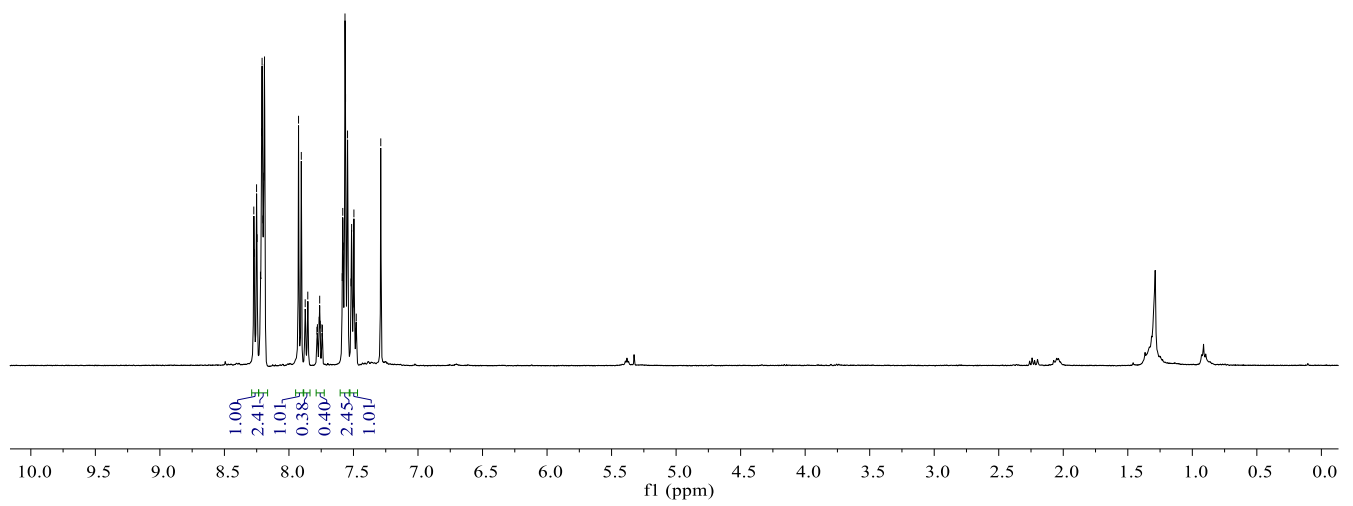




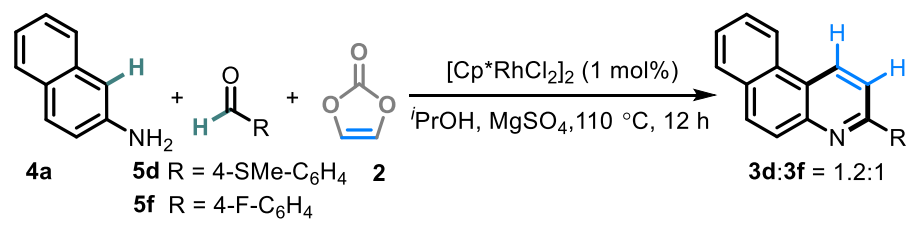

A pressure tube was charged with amine $4 \mathbf{a}(0.20 \mathrm{mmol})$, aldehyde $\mathbf{5 d}(0.20 \mathrm{mmol})$, aldehyde $5 \mathbf{f}(0.20 \mathrm{mmol})$, vinylene carbonate $2(0.20 \mathrm{mmol}),\left[\mathrm{Cp}^{*} \mathrm{RhCl}_{2}\right]_{2}(1.2 \mathrm{mg}, 0.002 \mathrm{mmol})$ and ${ }^{i} \mathrm{PrOH}$ $(2.0 \mathrm{~mL})$.. The reaction mixture was stirred at $110^{\circ} \mathrm{C}$ for $12 \mathrm{~h}$ under $\mathrm{Ar}$ atmosphere in an oil bath. After cooling to room temperature, all volatiles were removed under reduced pressure. The residue was purified by silica gel chromatography using PE/EA eluent to afford the products $\mathbf{3 d}$ (15.6 mg, 26\% yield) and $\mathbf{3 f}(12.1 \mathrm{mg}, 22 \%$ yield).

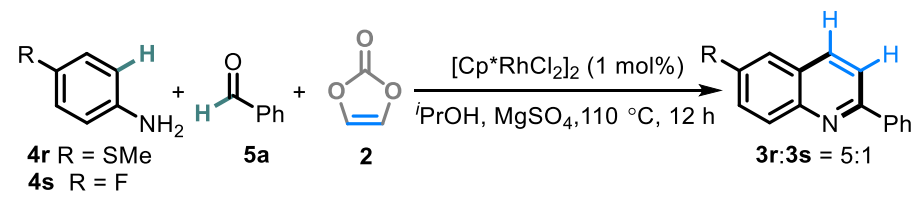

A pressure tube was charged with amine $4 \mathbf{r}(0.20 \mathrm{mmol})$, amine $4 \mathbf{s}(0.20 \mathrm{mmol})$, aldehyde $5 \mathbf{a}$ $(0.20 \mathrm{mmol})$, vinylene carbonate $2(0.20 \mathrm{mmol}),\left[\mathrm{Cp}^{*} \mathrm{RhCl}_{2}\right]_{2}(1.2 \mathrm{mg}, 0.002 \mathrm{mmol})$ and ${ }^{i} \mathrm{PrOH}$ $(2.0 \mathrm{~mL})$. The reaction mixture was stirred at $110{ }^{\circ} \mathrm{C}$ for $12 \mathrm{~h}$ under Ar atmosphere in an oil bath. After cooling to room temperature, all volatiles were removed under reduced pressure. The residue was purified by silica gel chromatography using PE/EA eluent to afford the products 3r (17.1 mg, 34\% yield) and $3 \mathrm{~s}$ ( $3.2 \mathrm{mg}, 7 \%$ yield).

\section{F. References}

(1) Tang, Q.; Fu, W.; Zhang, M.; Wang, E.; Shan, L.; Chai, X.; Pang, J.; Wang, X.; Xu, X.; Xu, L.; Li, D.; Sheng, R.; Hou, T. Eur. J. Org. Chem. 2020, 192, 112156.

(2) Luo, H.; Chen, K.; Jiang, H.; Zhu, S. Org. Lett. 2016, 18, 5208.

(3) Kozlov, N. G.; Basalaeva, L. I.; Skakovskaya, Yu. E. Russ. J. Gen. Chem. 2002, 72, 1238.

(4) Liu, L.; Zhang, S.; Fu, X.; Yan, C. H. Chem. Commun. 2011, 47, 10148.

(5) Xi, Z. W.; Yang, L.; Wang, D. Y.; Pu, C. D.; Shen, Y. M.; Wu, C. D.; Peng X. G. J. Org. Chem. 2018, 83, 11886.

(6) Hong, X.; Wang, H.; Liu, B.; Xu, B. Chem. Commun. 2014, 50, 14129. 


\section{G. NMR spectra}

${ }^{1} \mathrm{H}$ NMR of 3a $\left(400 \mathrm{MHz}, \mathrm{CDCl}_{3}\right)$

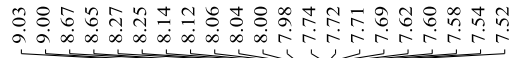

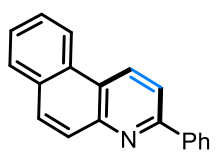

$3 \mathbf{a}$

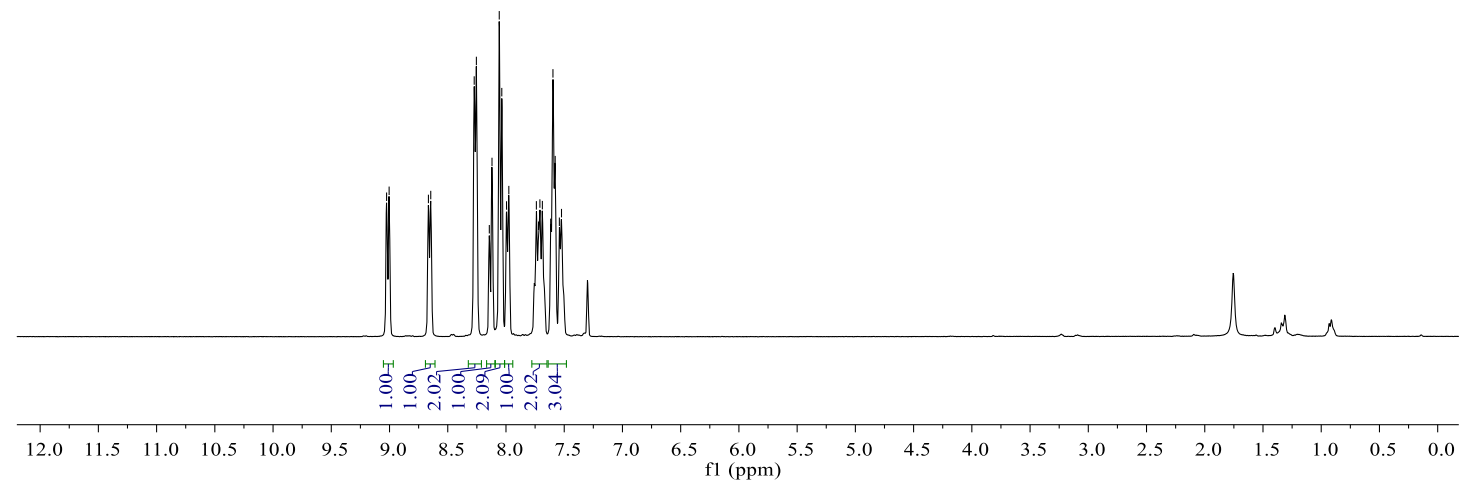

${ }^{13} \mathrm{C}$ NMR of 3a $\left(100 \mathrm{MHz}, \mathrm{CDCl}_{3}\right)$

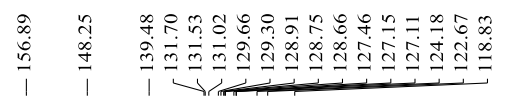

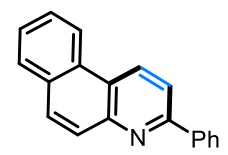

$3 a$

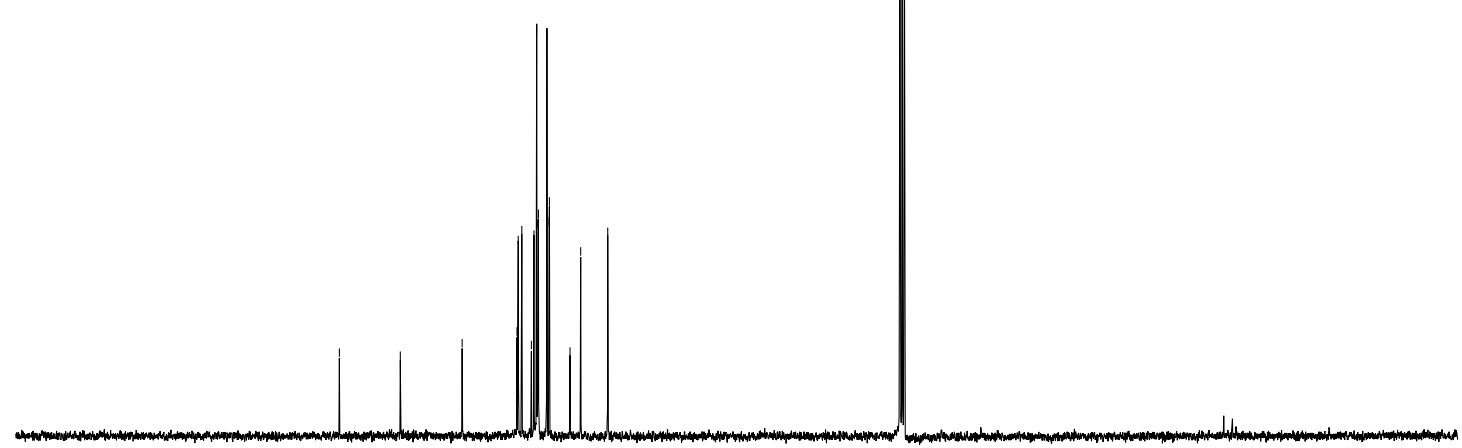

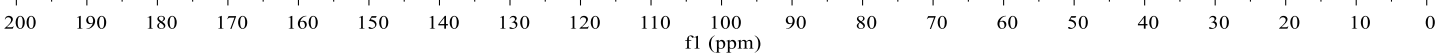


${ }^{1} \mathrm{H}$ NMR of $\mathbf{3 b}\left(400 \mathrm{MHz}, \mathrm{CDCl}_{3}\right)$

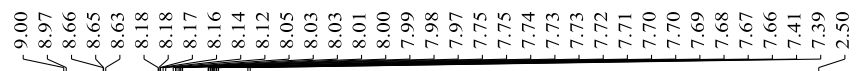
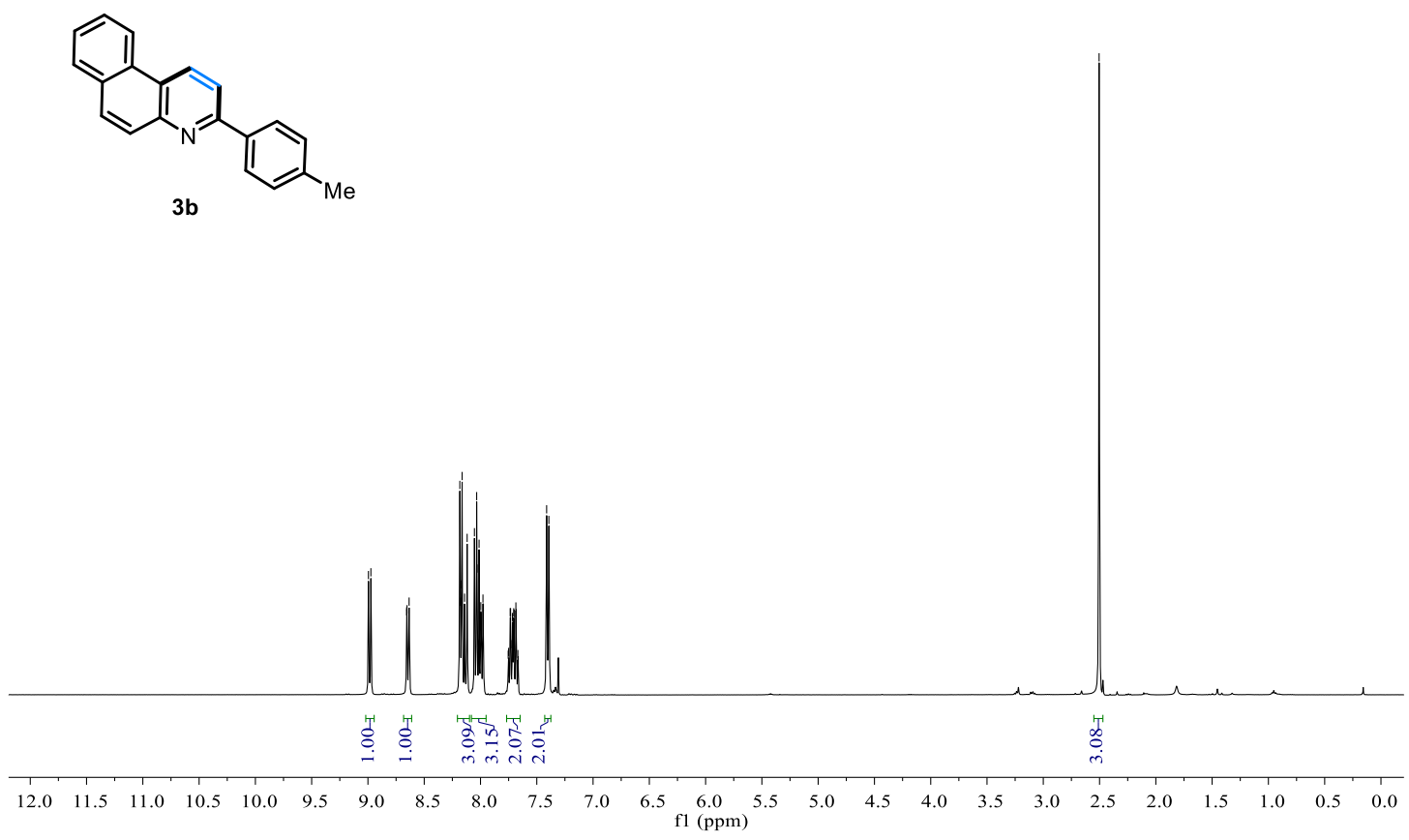

${ }^{13} \mathrm{C}$ NMR of $\mathbf{3 b}\left(100 \mathrm{MHz}, \mathrm{CDCl}_{3}\right)$

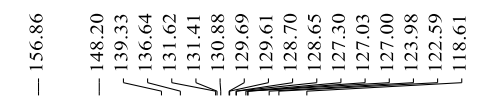

$\stackrel{\infty}{\stackrel{\infty}{1}}$
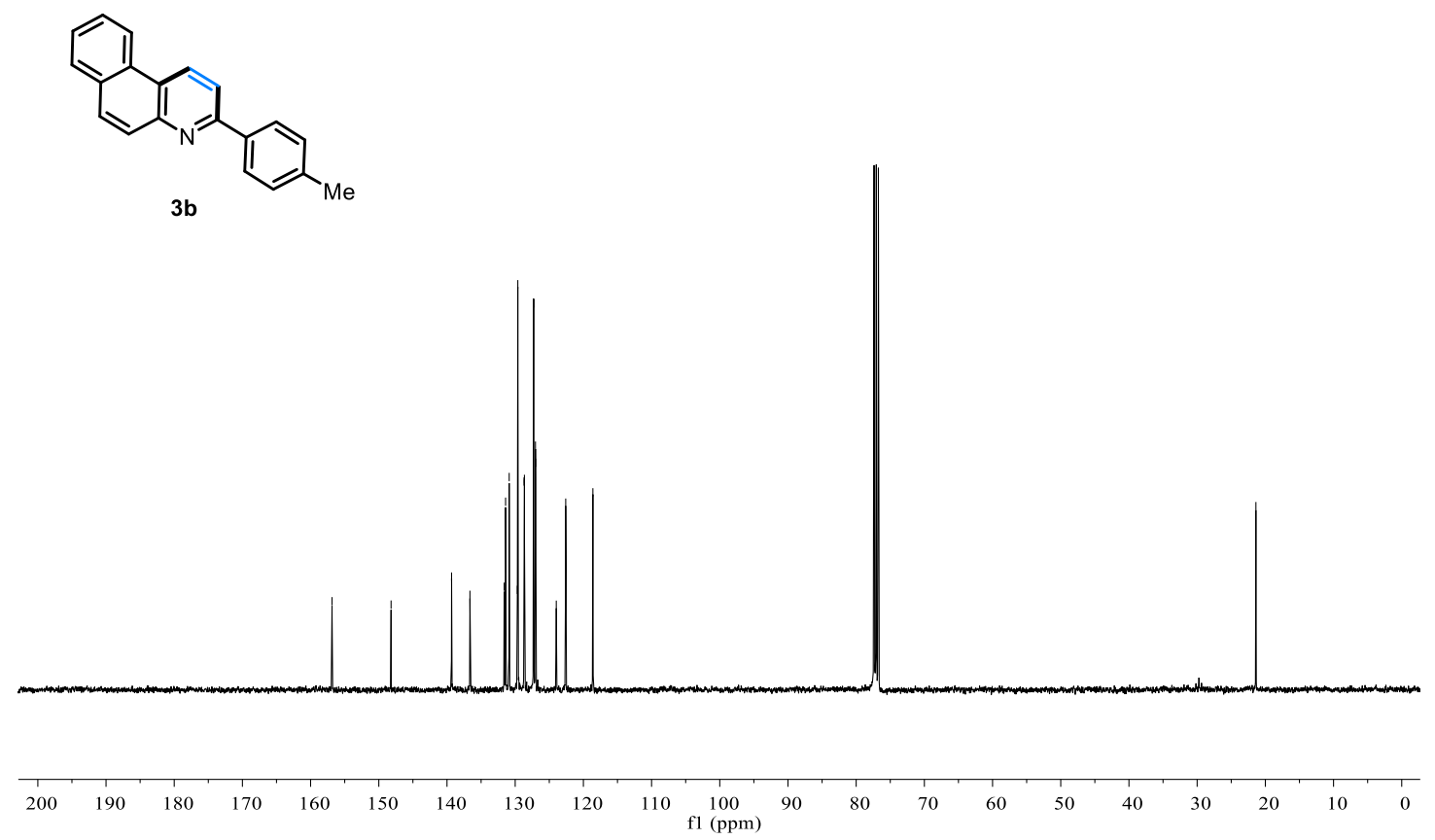

S20 
${ }^{1} \mathrm{H} \mathrm{NMR}$ of $3 \mathbf{c}\left(400 \mathrm{MHz}, \mathrm{CDCl}_{3}\right)$

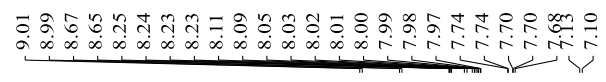

$\stackrel{n}{\infty}$
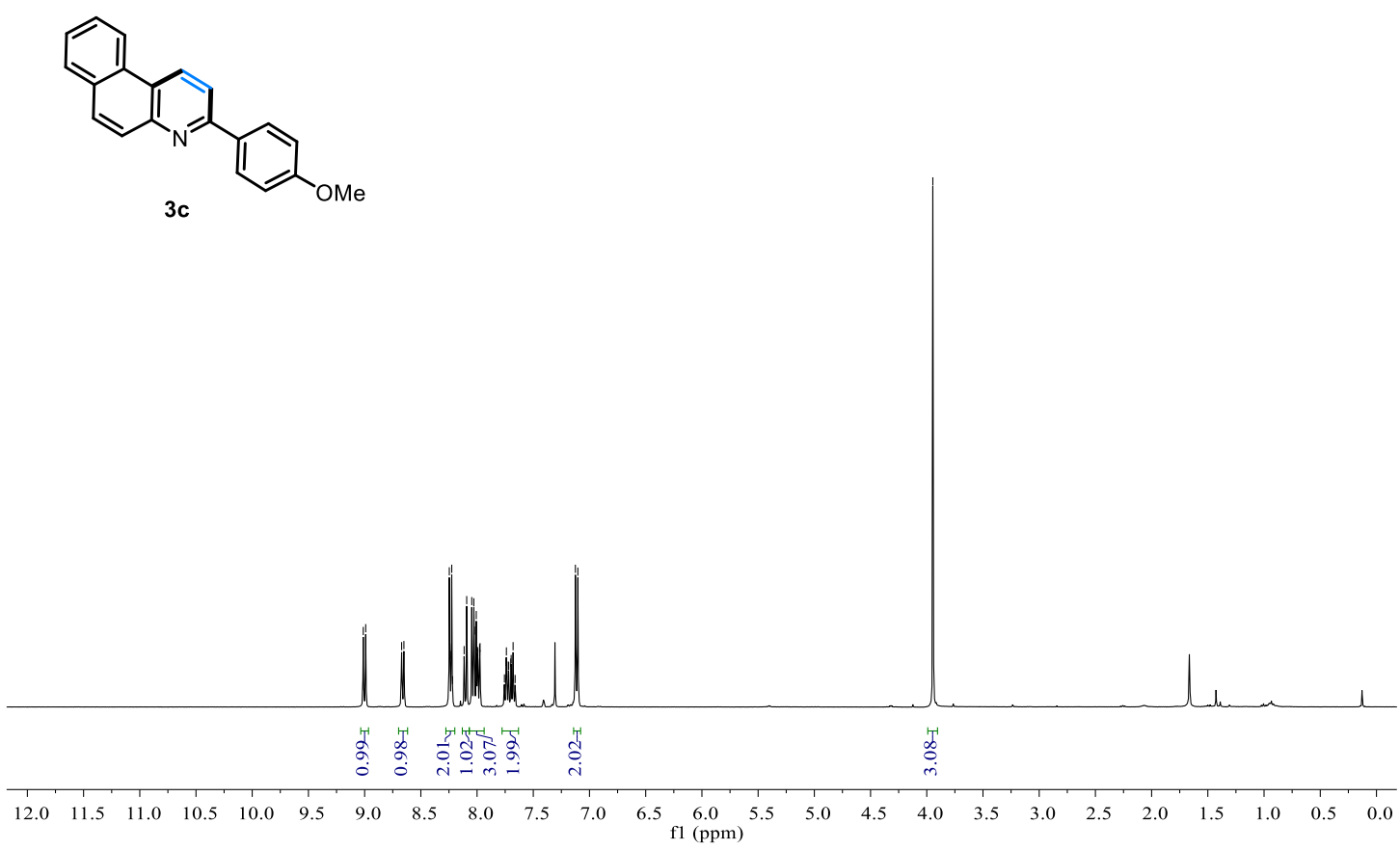

${ }^{13} \mathrm{C} \mathrm{NMR}$ of $3 \mathbf{c}\left(100 \mathrm{MHz}, \mathrm{CDCl}_{3}\right)$

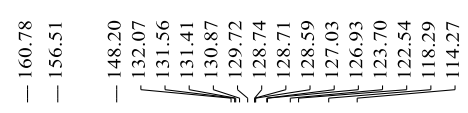

7
0
0
1

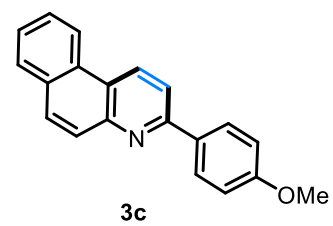

$3 c$
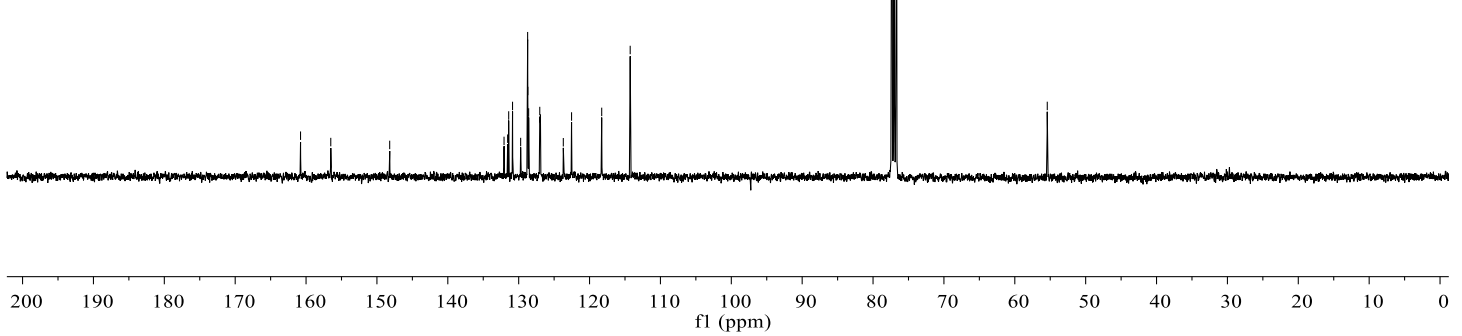

S21 
${ }^{1} \mathrm{H}$ NMR of $\mathbf{3 d}\left(400 \mathrm{MHz}, \mathrm{CDCl}_{3}\right)$

$\underbrace{\underbrace{}_{0}}$
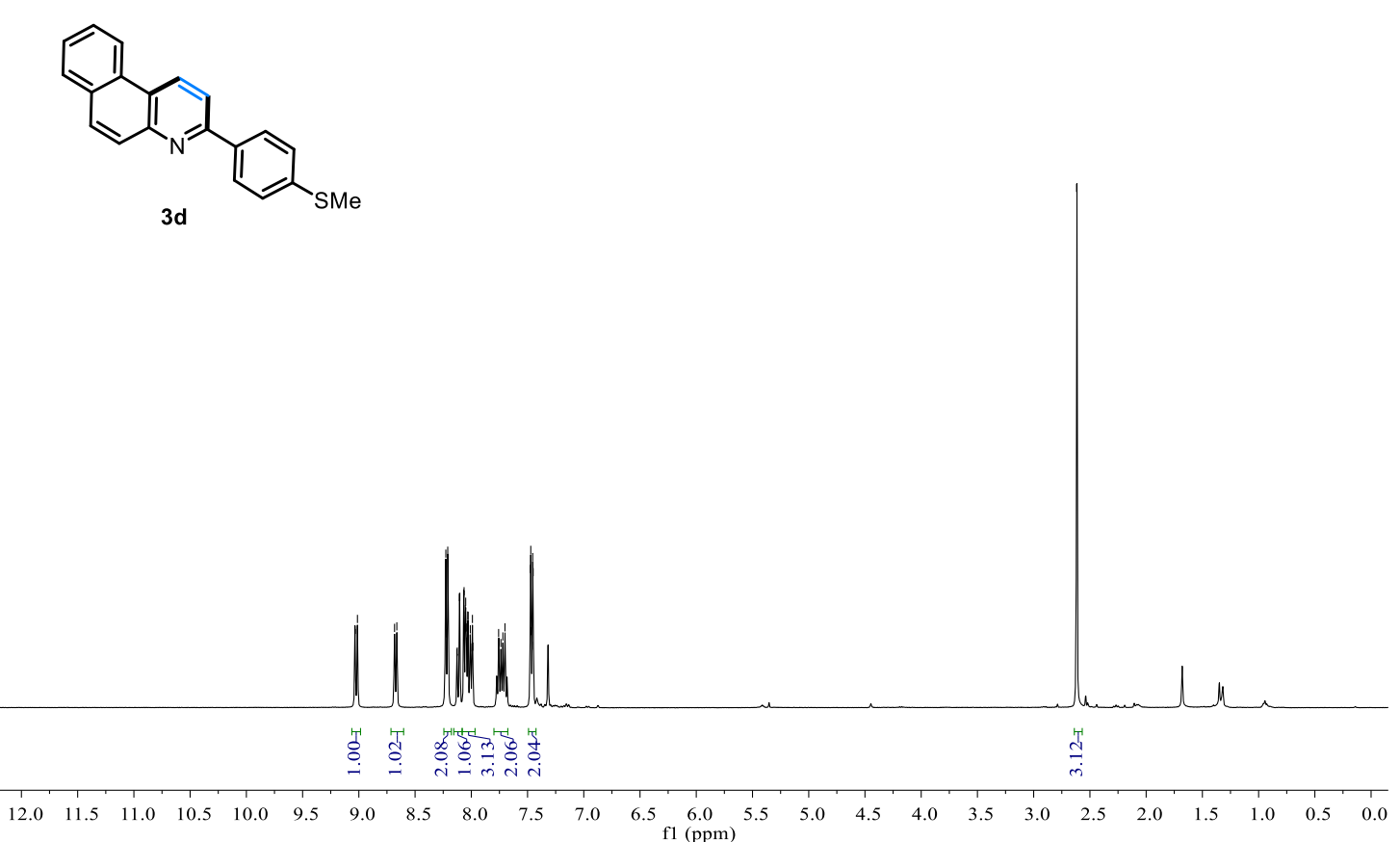

${ }^{13} \mathrm{C}$ NMR of Bd $\left(100 \mathrm{MHz}, \mathrm{CDCl}_{3}\right)$
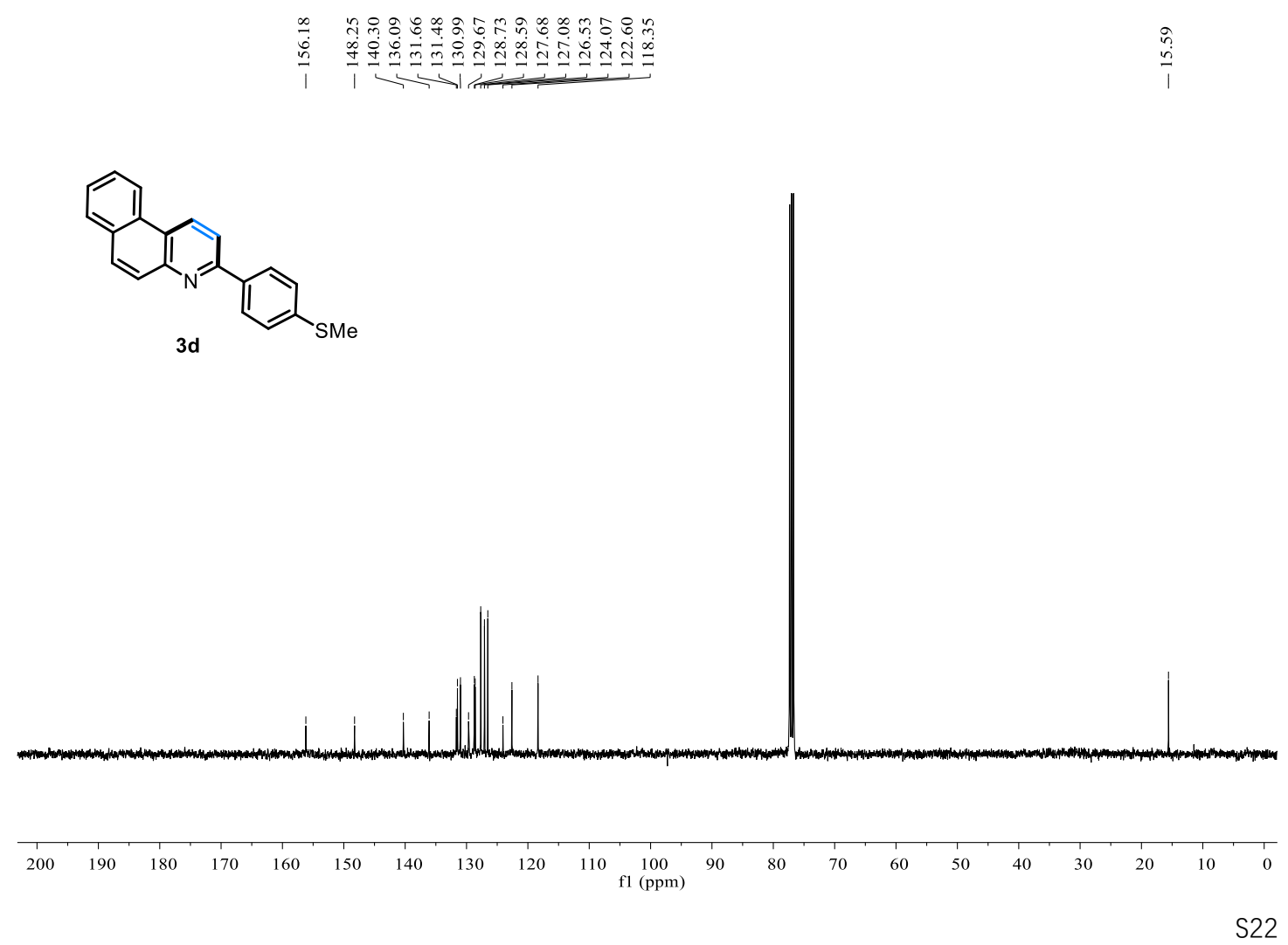
${ }^{1} \mathrm{H} \mathrm{NMR}$ of $\mathbf{3 e}\left(400 \mathrm{MHz}, \mathrm{CDCl}_{3}\right)$

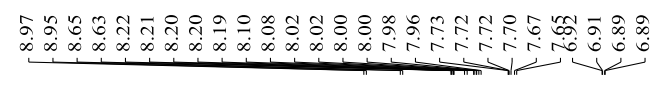

$\stackrel{\circ}{\stackrel{2}{i}}$
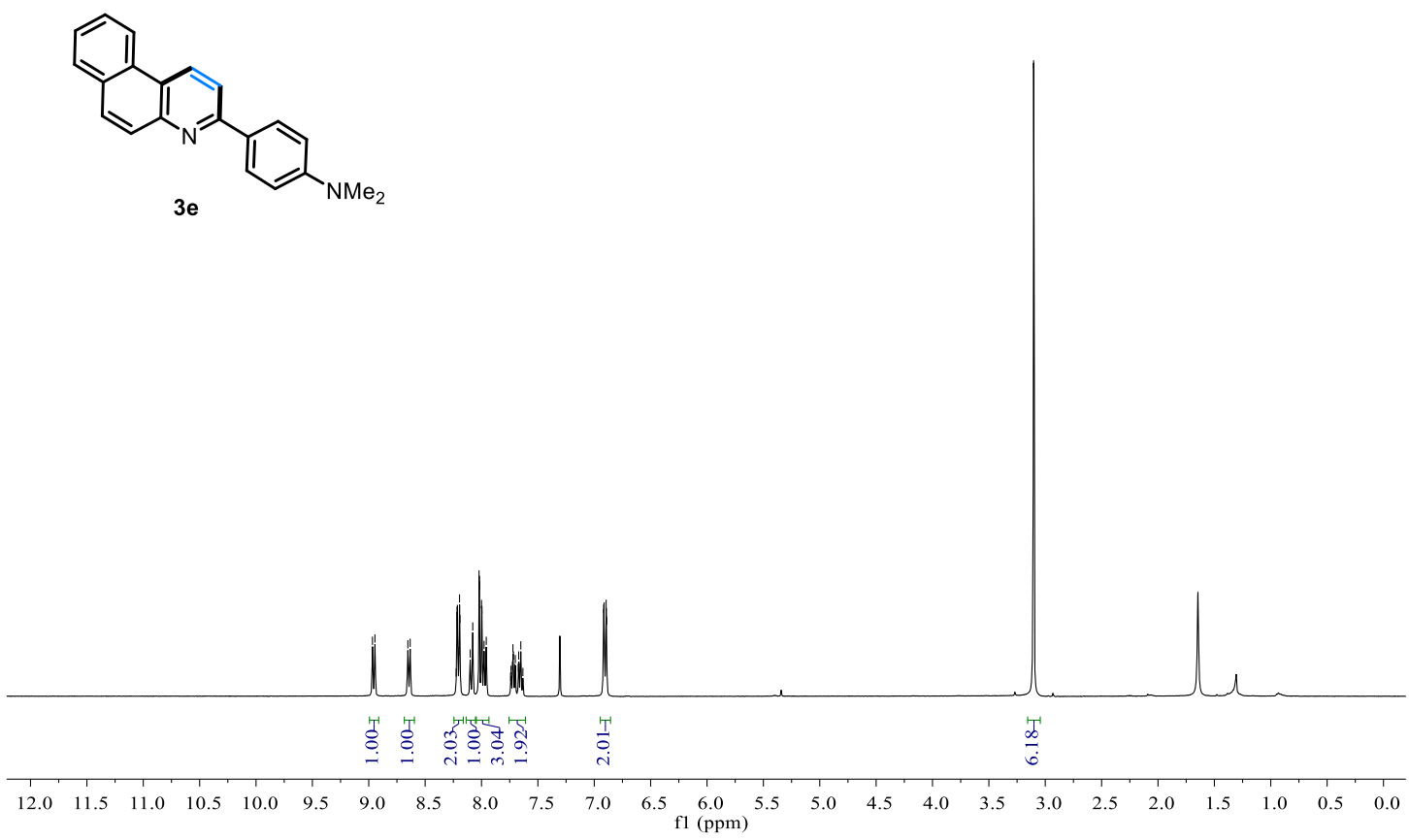

${ }^{13} \mathrm{C} \mathrm{NMR}$ of $3 \mathbf{e}\left(100 \mathrm{MHz}, \mathrm{CDCl}_{3}\right)$

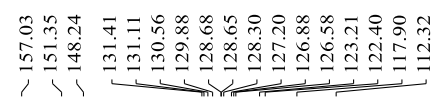

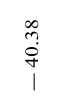

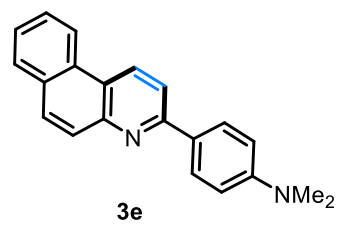

$3 e$
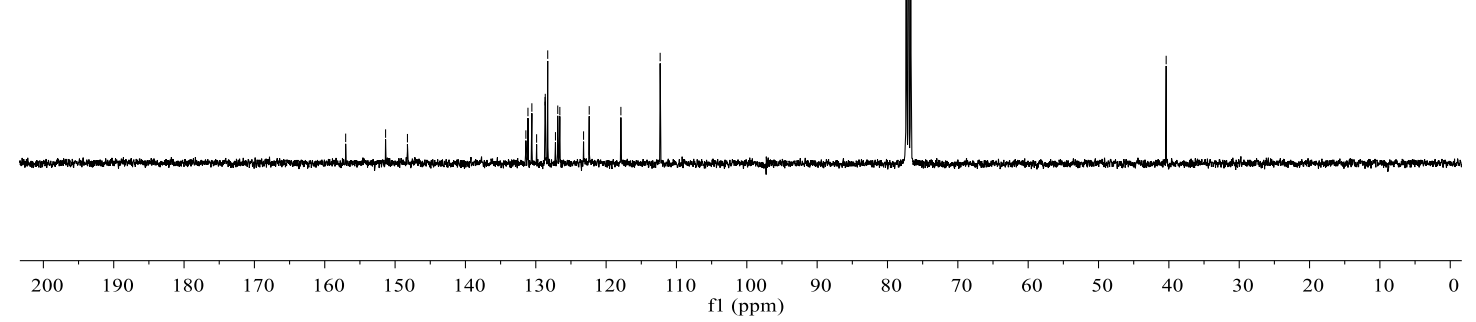

S23 
${ }^{1} \mathrm{H} \mathrm{NMR}$ of $\mathbf{3 f}\left(400 \mathrm{MHz}, \mathrm{CDCl}_{3}\right)$

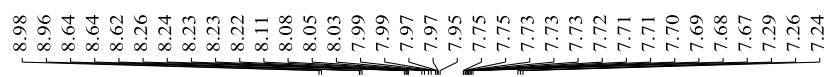
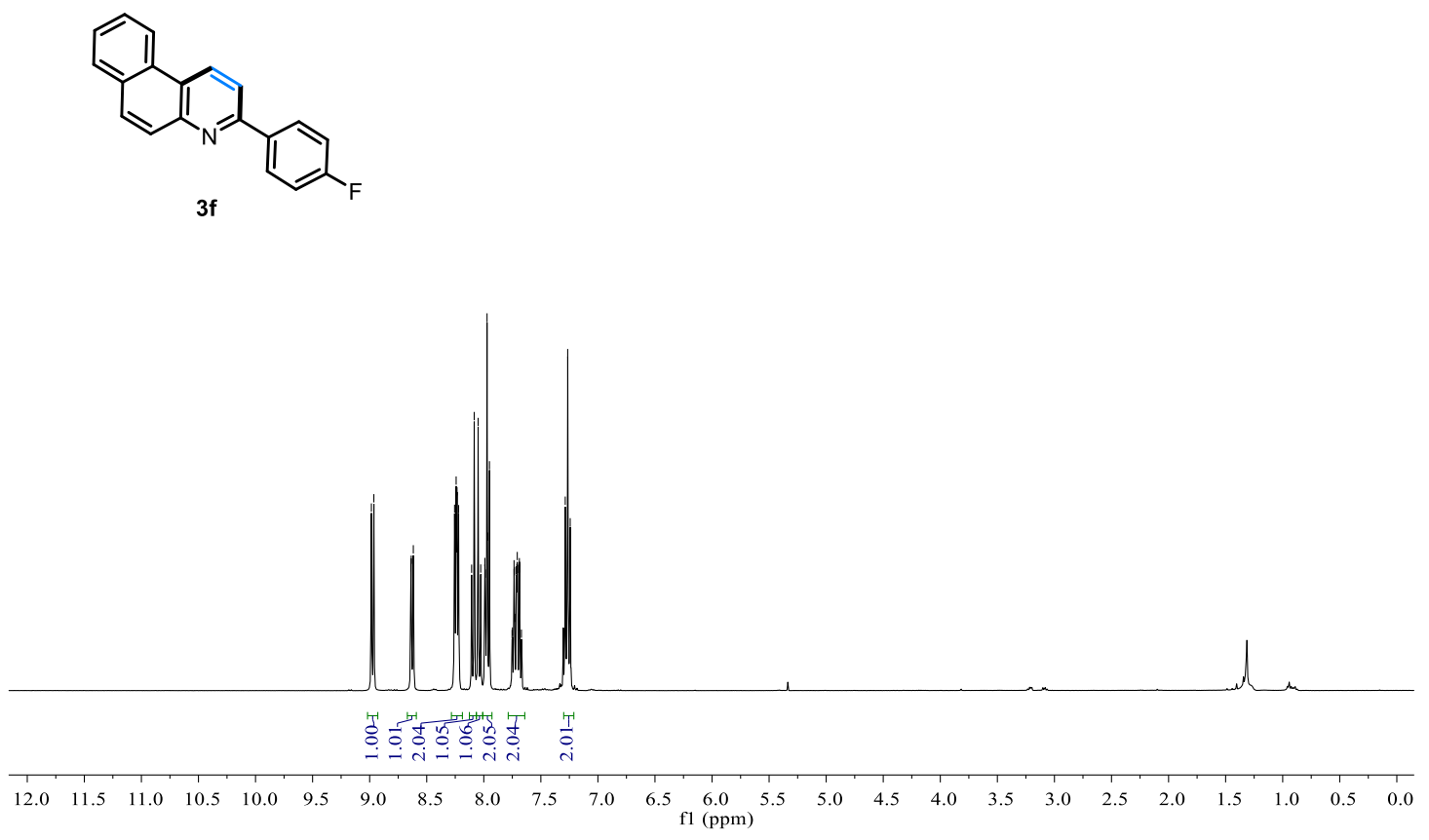

${ }^{13} \mathrm{C} \mathrm{NMR}$ of $3 \mathbf{f}\left(100 \mathrm{MHz}, \mathrm{CDCl}_{3}\right)$

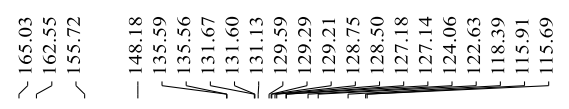
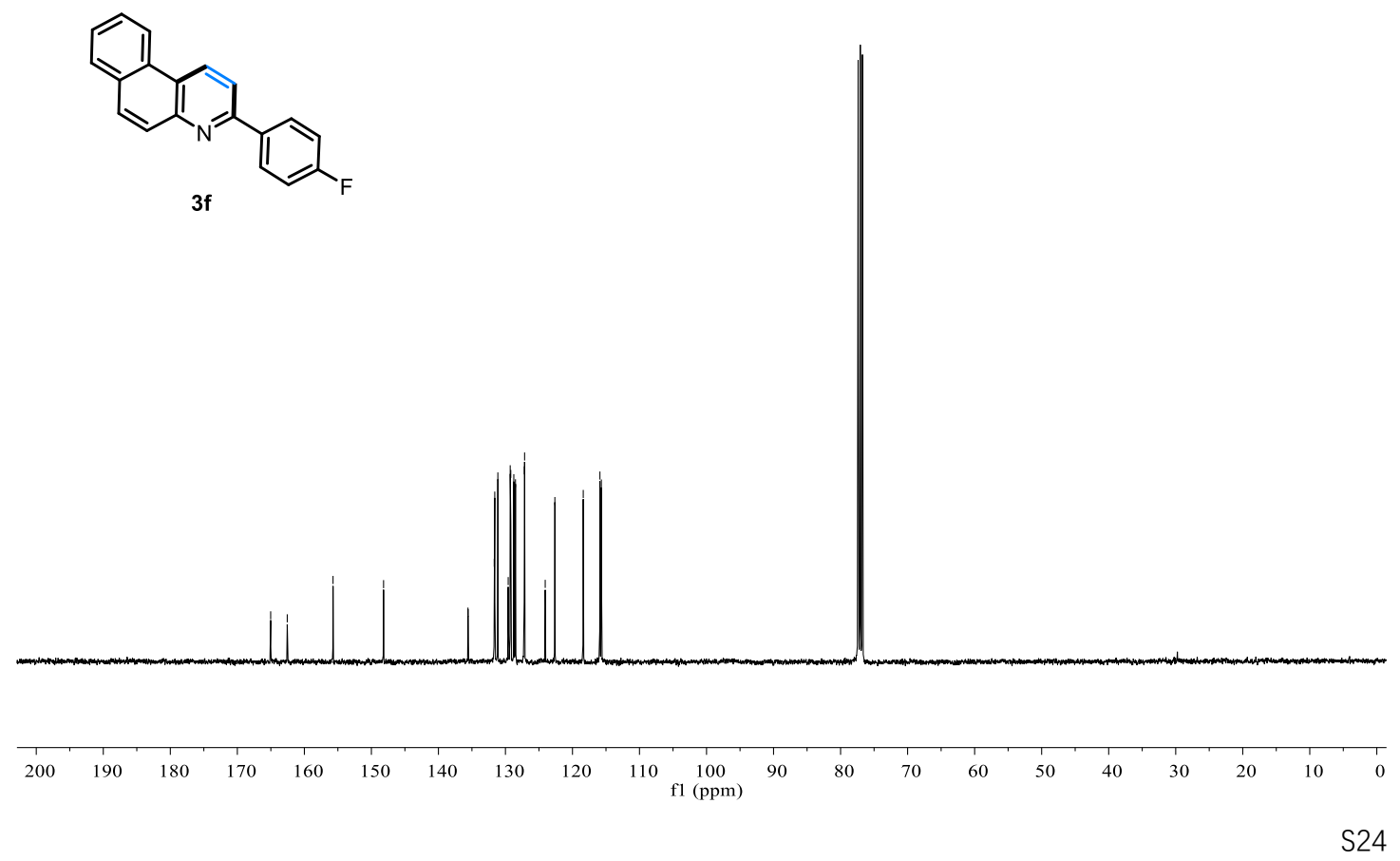
${ }^{19} \mathrm{~F}$ NMR of $\mathbf{3 f}\left(376 \mathrm{MHz}, \mathrm{CDCl}_{3}\right)$

$\stackrel{\frac{j}{i}}{i}$
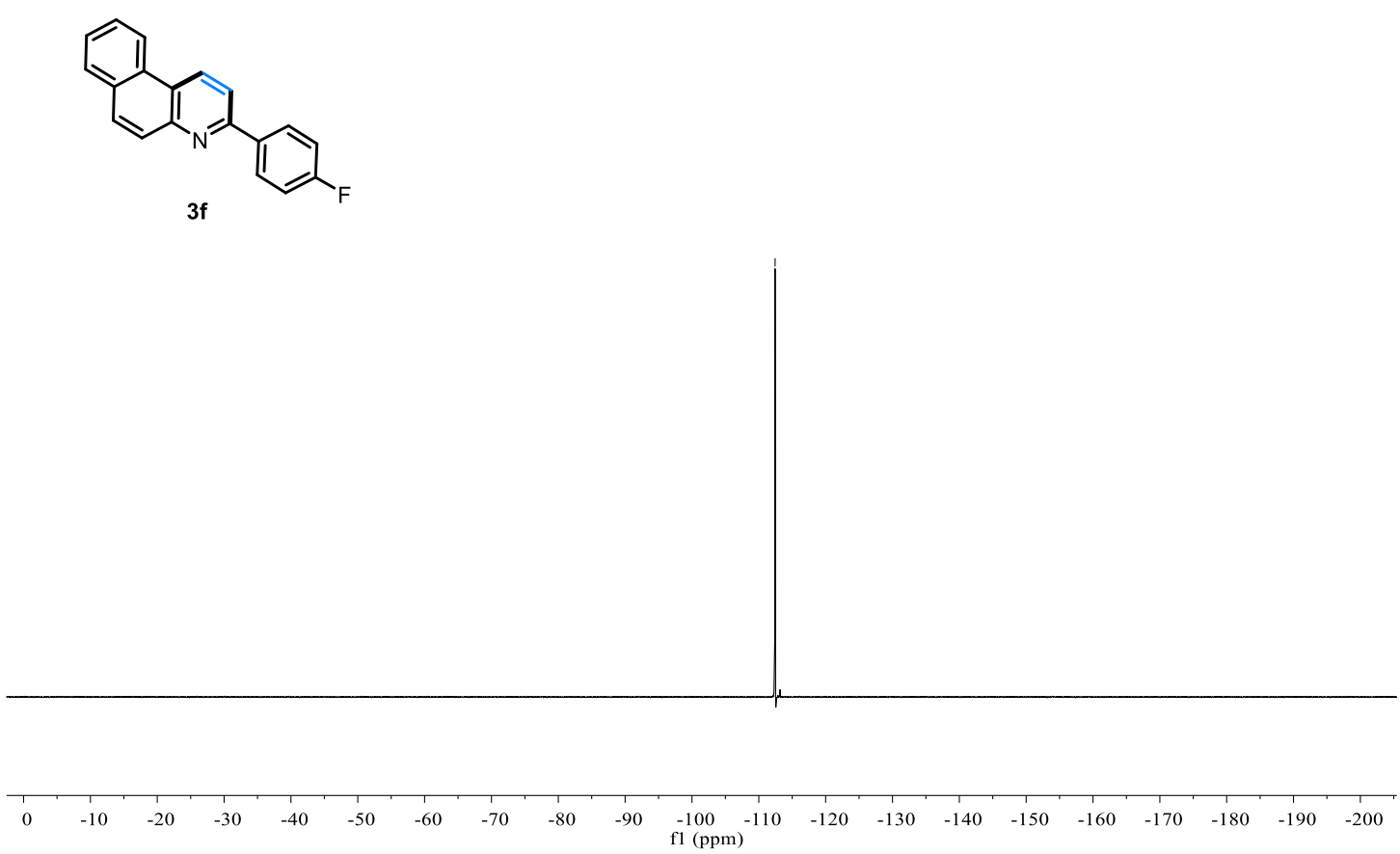

${ }^{1} \mathrm{H}$ NMR of $\mathbf{3 g}\left(400 \mathrm{MHz}, \mathrm{CDCl}_{3}\right)$

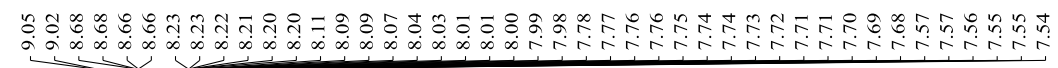
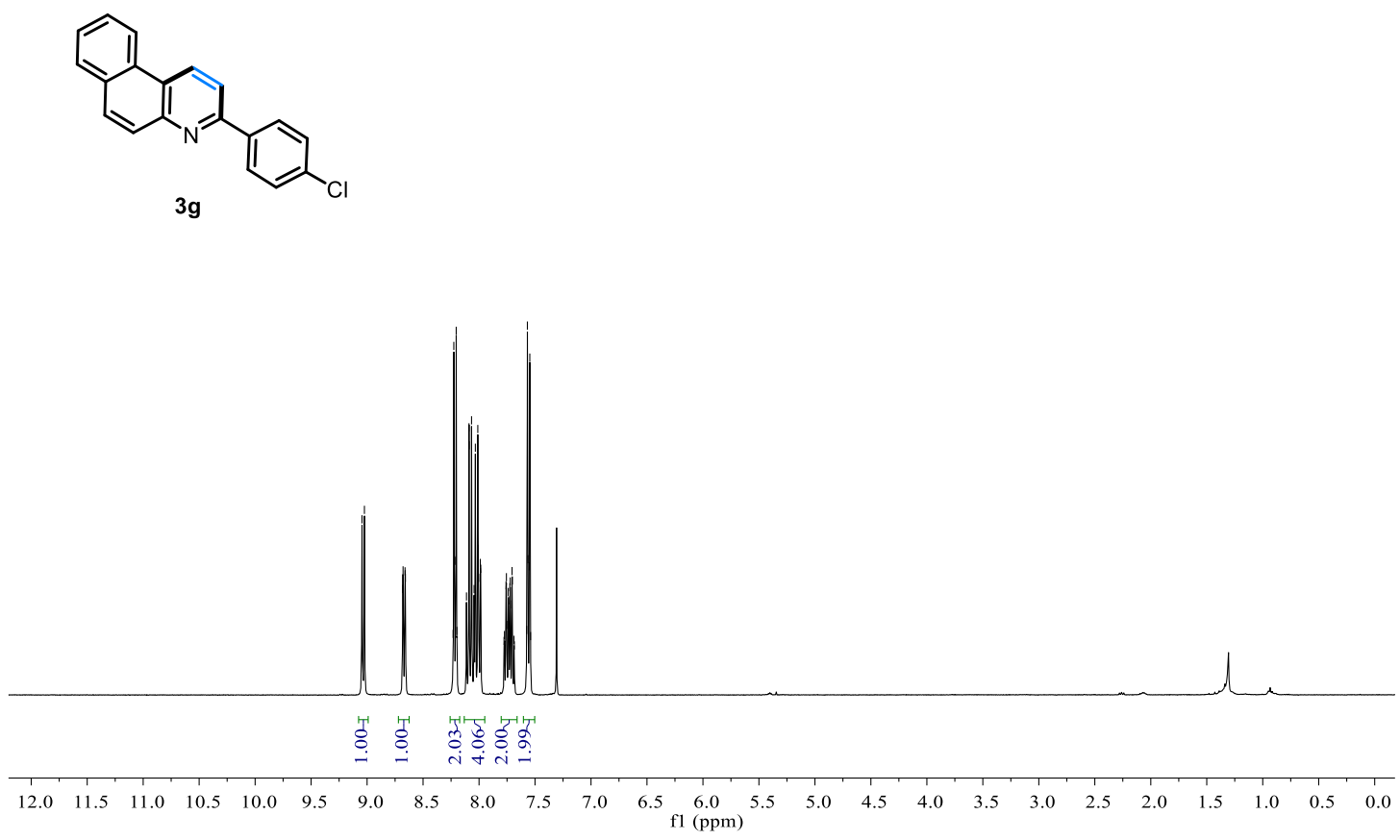

S25 
${ }^{13} \mathrm{C} \mathrm{NMR}$ of $\mathbf{3 g}\left(100 \mathrm{MHz}, \mathrm{CDCl}_{3}\right)$
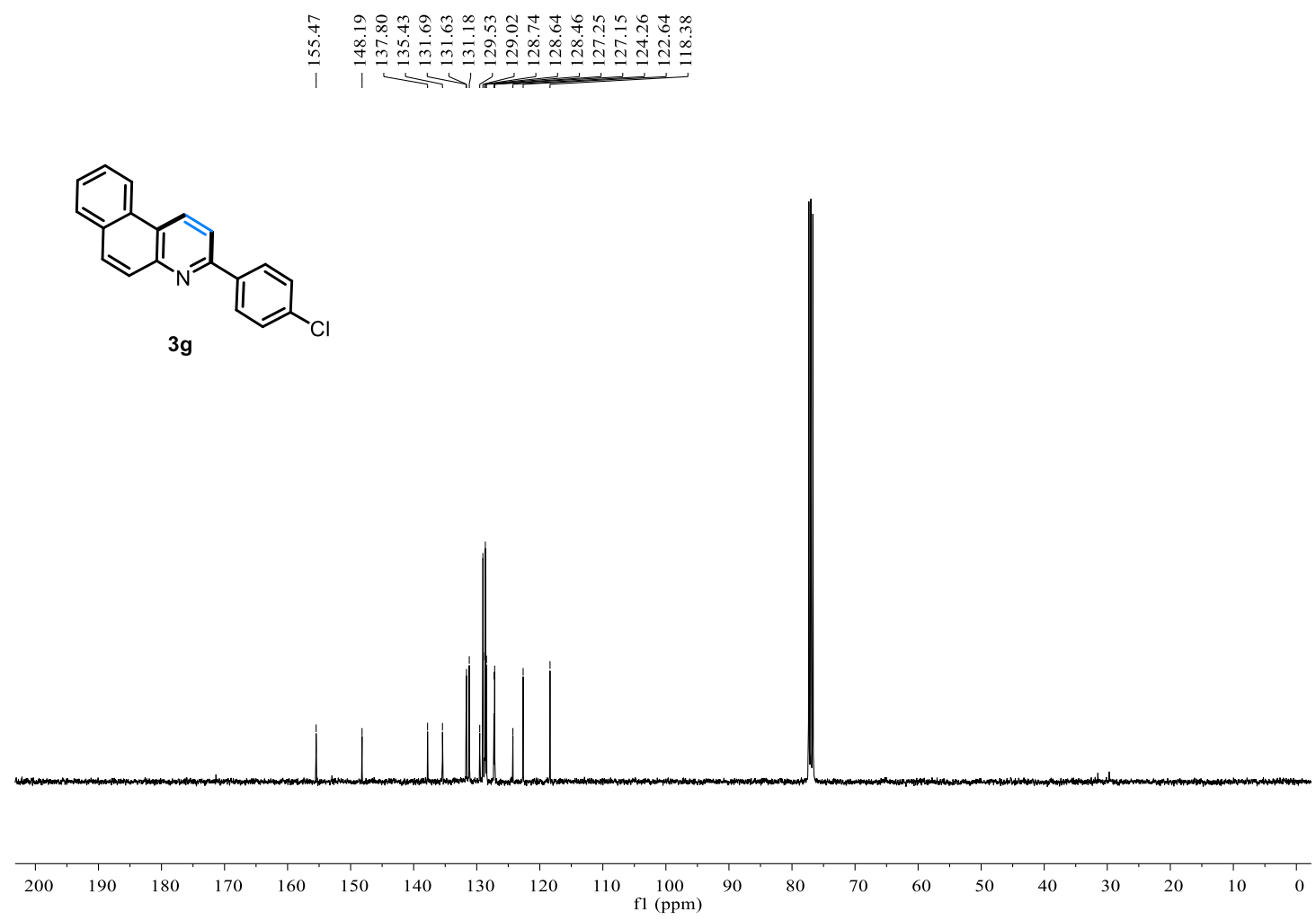

${ }^{1} \mathrm{H}$ NMR of $3 \mathbf{h}\left(400 \mathrm{MHz}, \mathrm{CDCl}_{3}\right)$

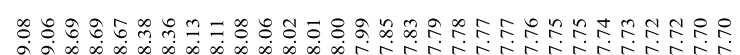
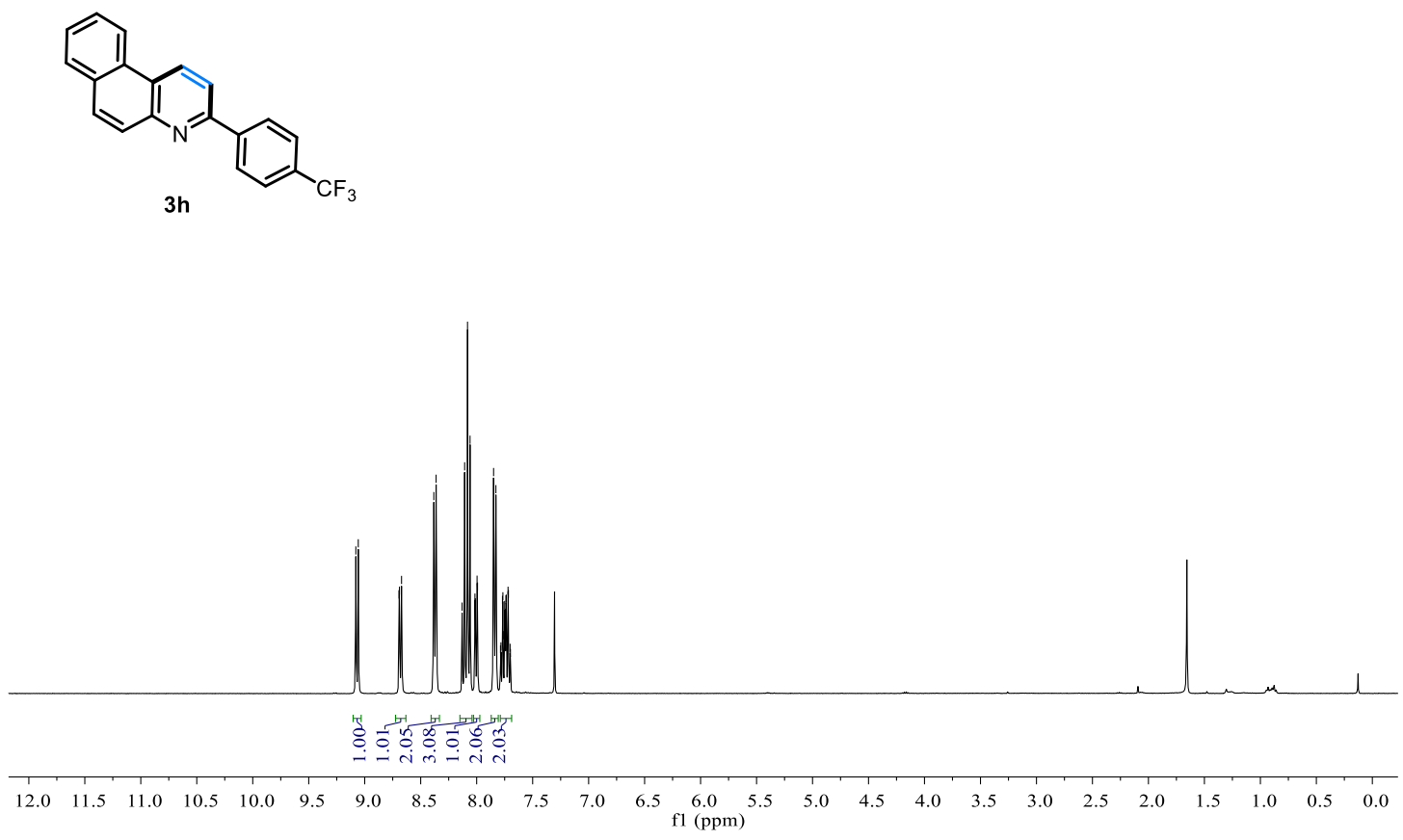

S26 
${ }^{13} \mathrm{C}$ NMR of $\mathbf{3 h}\left(100 \mathrm{MHz}, \mathrm{CDCl}_{3}\right)$

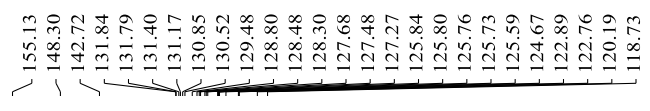

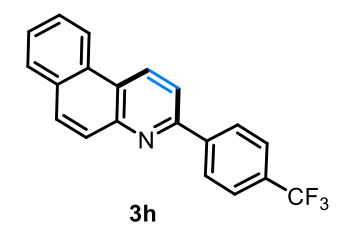

$3 h$
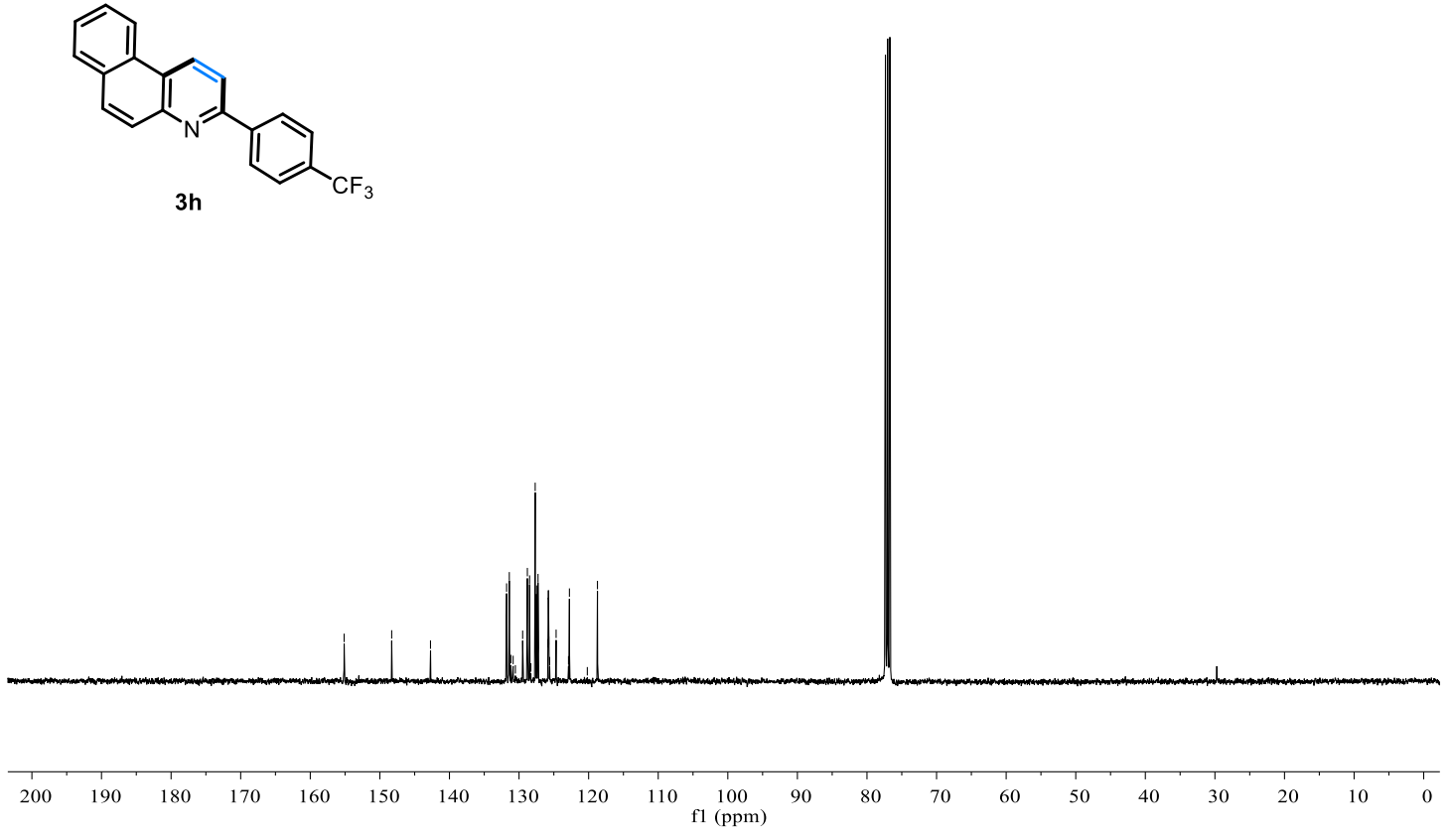

${ }^{19} \mathrm{~F}$ NMR of $\mathbf{3 h}\left(376 \mathbf{M H z}, \mathrm{CDCl}_{3}\right)$

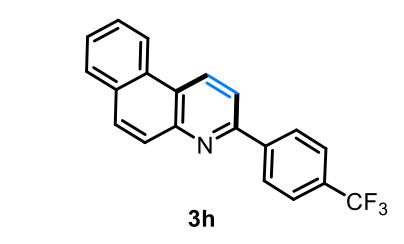

$3 h$

id

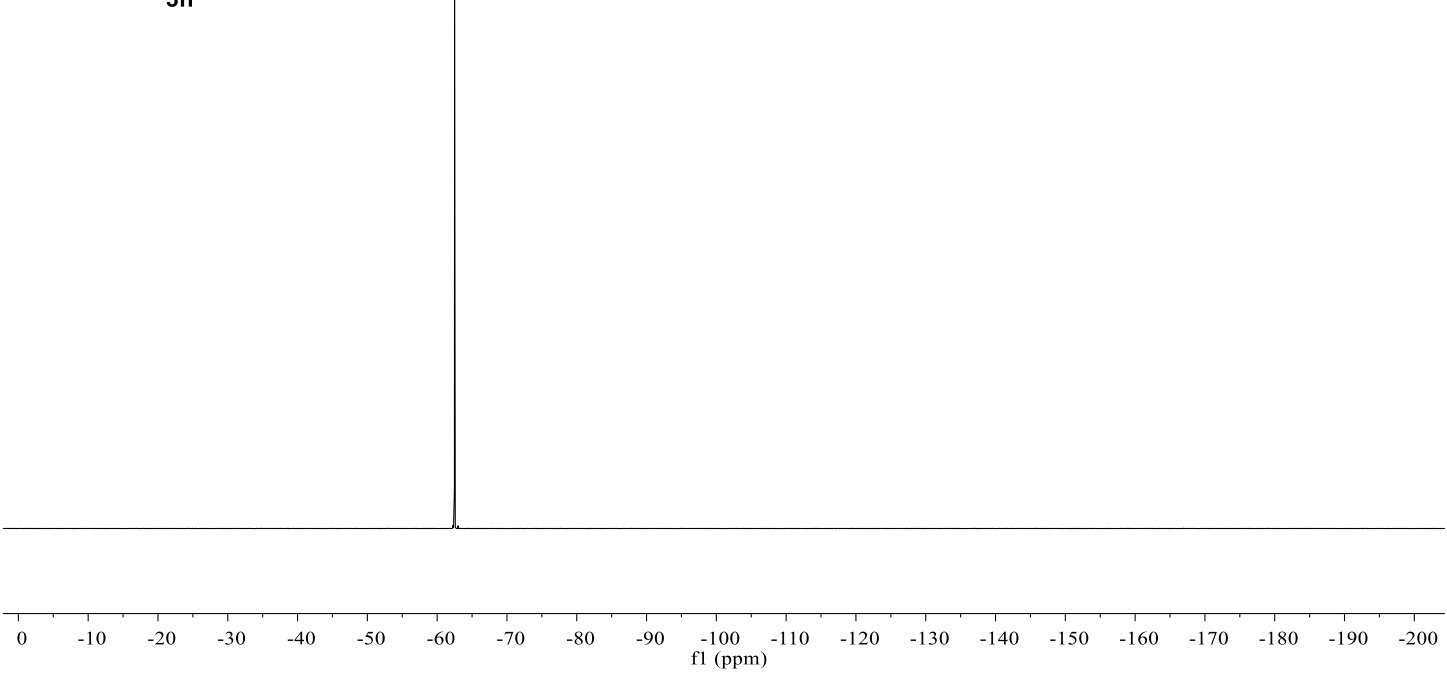

S27 
${ }^{1} \mathrm{H} \mathrm{NMR}$ of $3 \mathbf{i}\left(400 \mathrm{MHz}, \mathrm{CDCl}_{3}\right)$

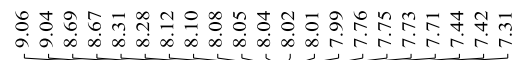

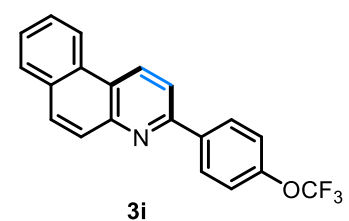

$3 \mathbf{i}$

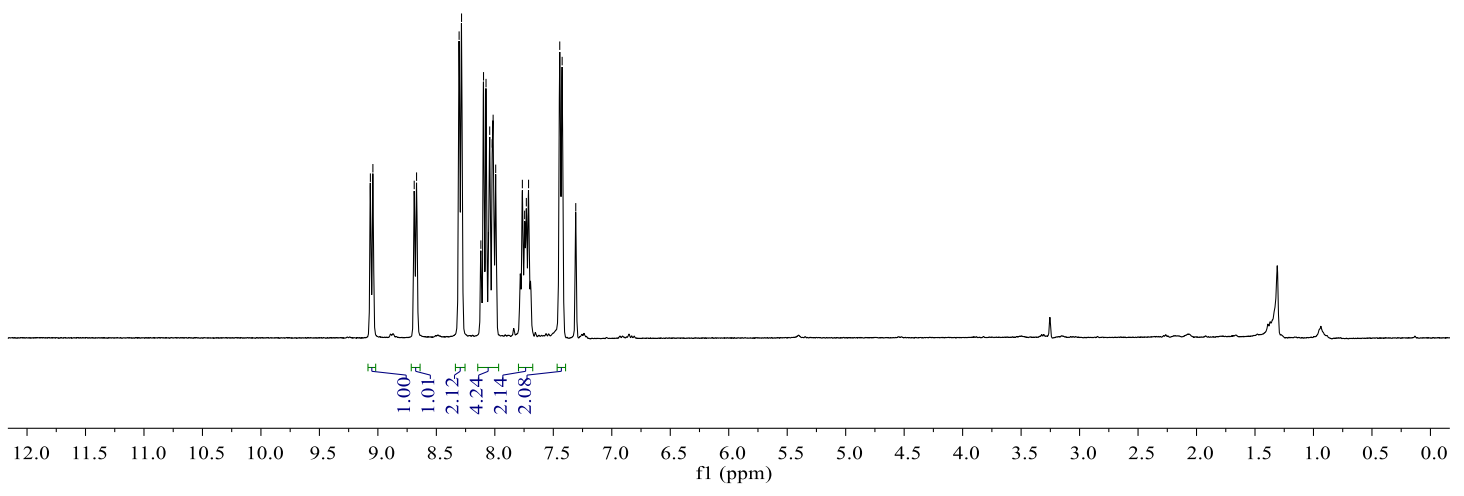

${ }^{13} \mathrm{C}$ NMR of $3 \mathbf{i}\left(100 \mathrm{MHz}, \mathrm{CDCl}_{3}\right)$

等

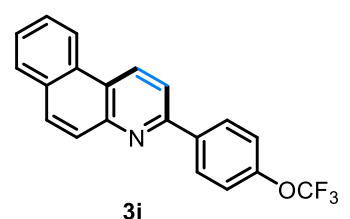

$3 \mathbf{i}$
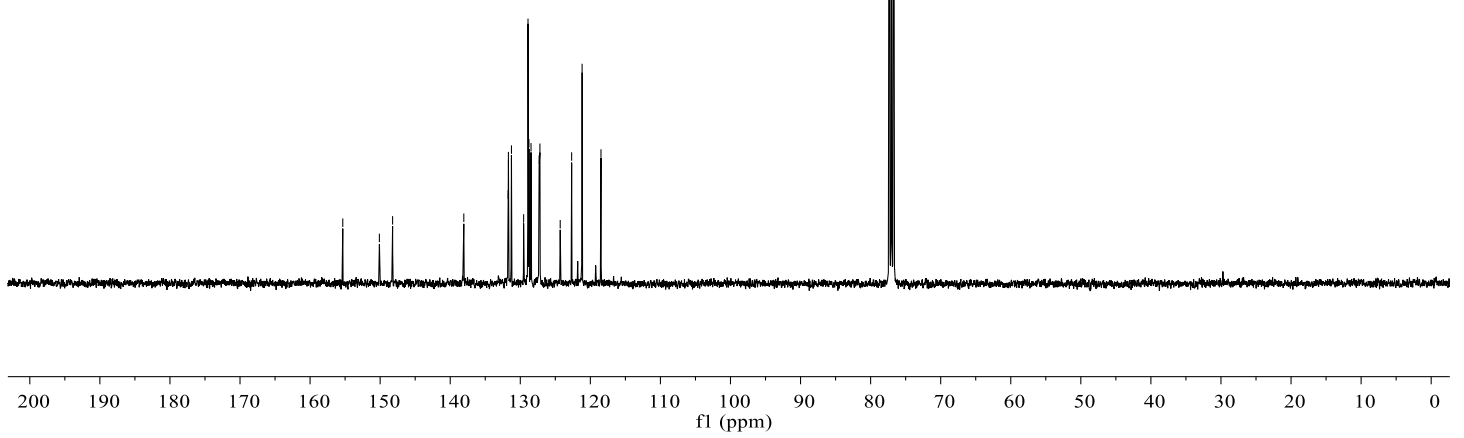

S28 
${ }^{19} \mathrm{~F}$ NMR of $3 \mathbf{i}\left(376 \mathrm{MHz}, \mathrm{CDCl}_{3}\right)$

in

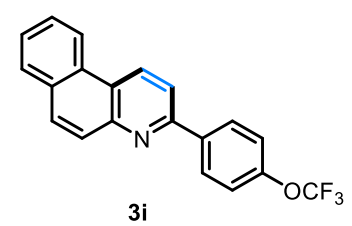

$3 \mathbf{i}$

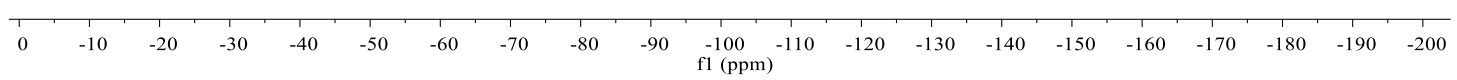

${ }^{1} \mathrm{H}$ NMR of $\mathbf{3 j}\left(400 \mathrm{MHz}, \mathrm{CDCl}_{3}\right)$

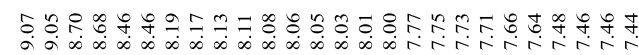

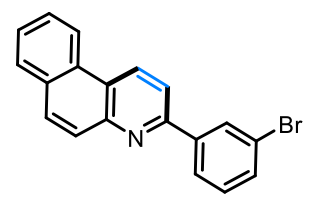

jj

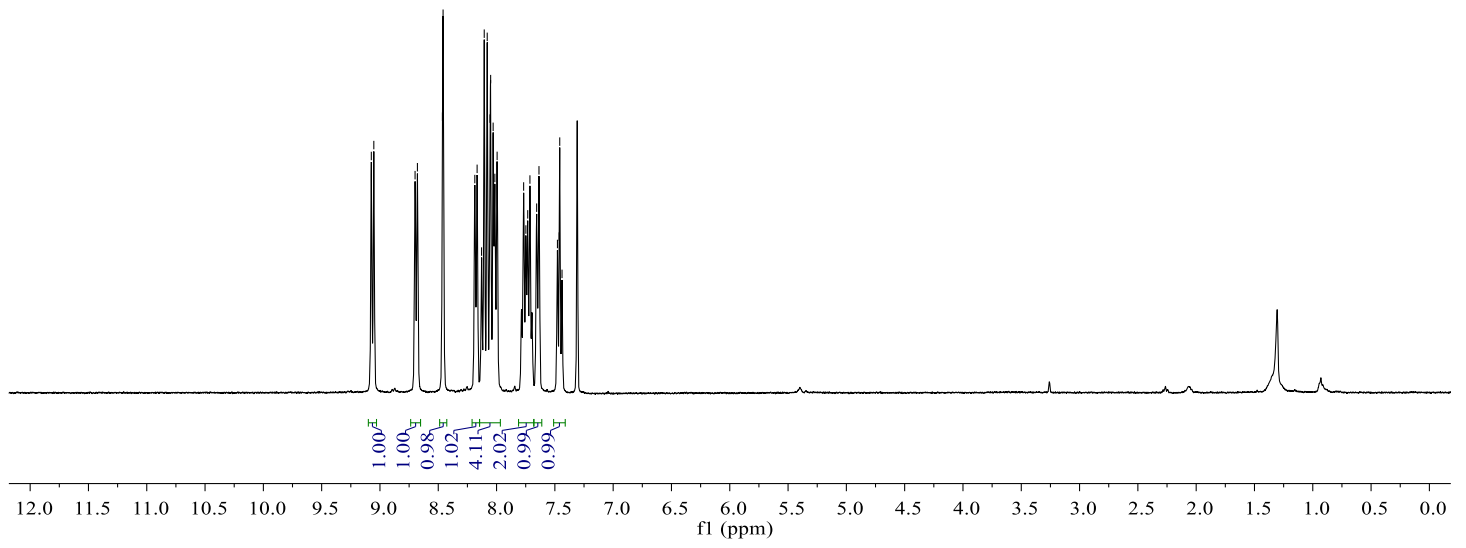

S29 
${ }^{13} \mathrm{C}$ NMR of $3 \mathbf{j}$ (100 MHz, $\left.\mathrm{CDCl}_{3}\right)$

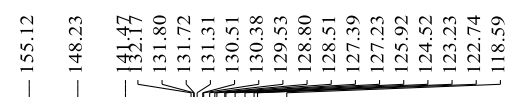

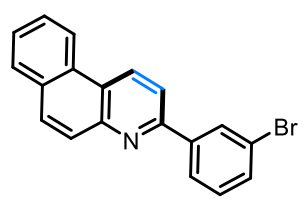

jj
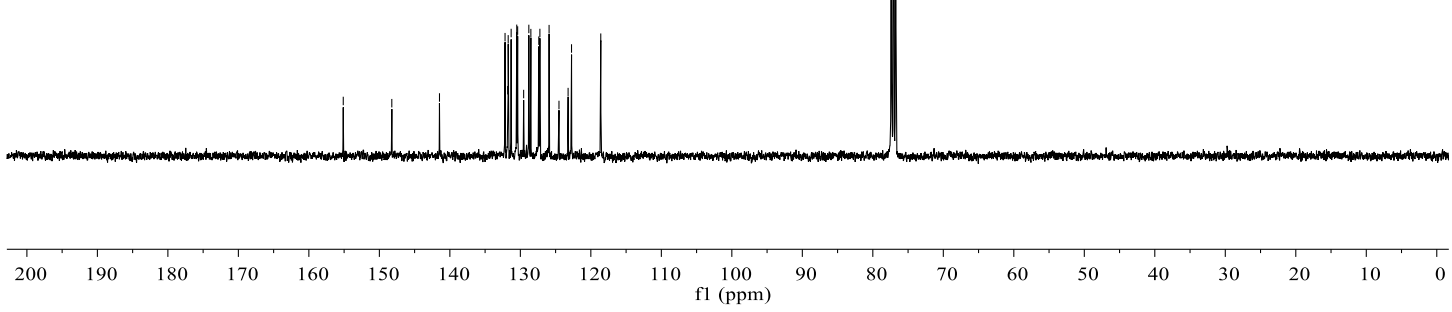

${ }^{1} \mathrm{H}$ NMR of $3 \mathbf{k}\left(400 \mathrm{MHz}, \mathrm{CDCl}_{3}\right)$

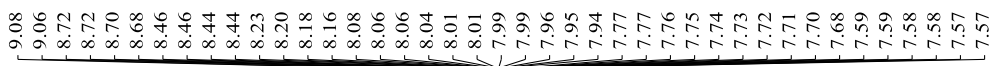
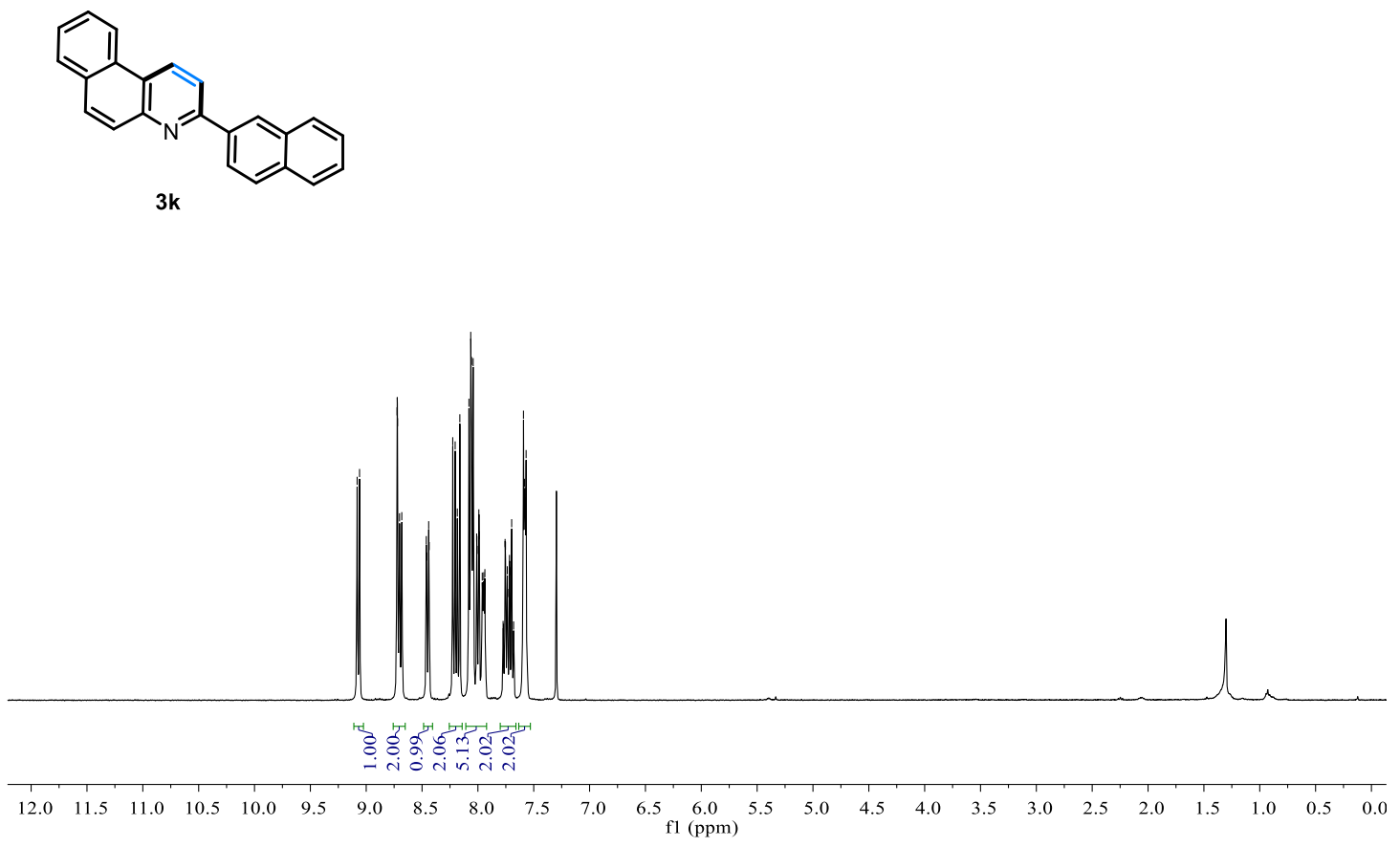

S30 
${ }^{13} \mathrm{C}$ NMR of $3 \mathbf{k}\left(100 \mathrm{MHz}, \mathrm{CDCl}_{3}\right)$

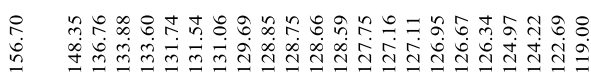

(1)

3k
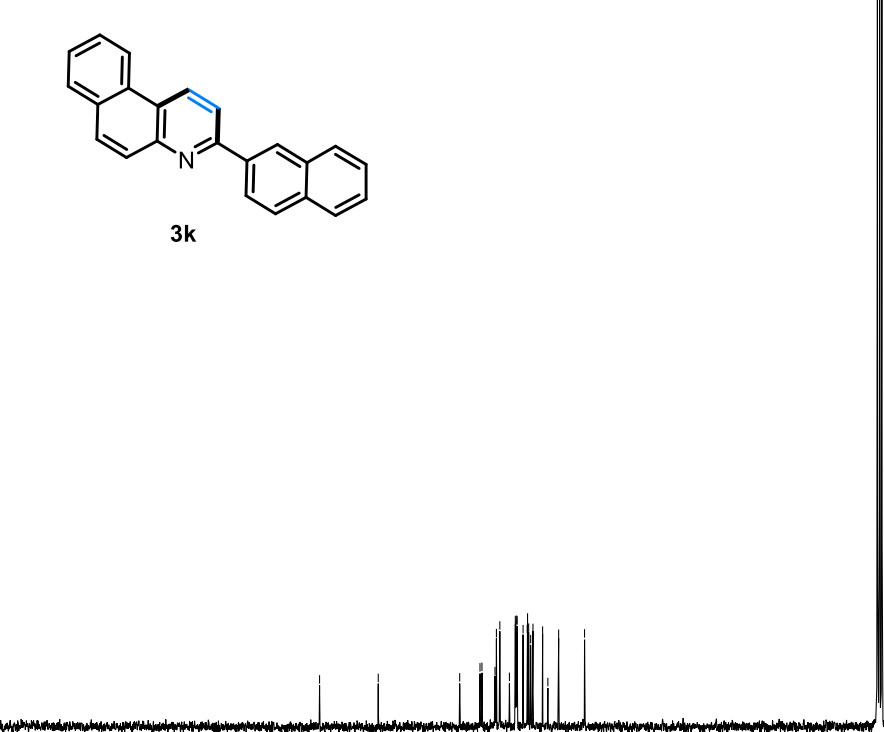

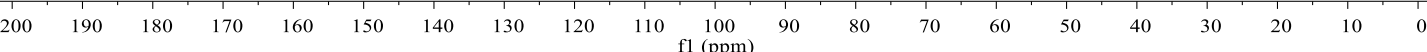

${ }^{1} \mathrm{H}$ NMR of $31\left(400 \mathrm{MHz}, \mathrm{CDCl}_{3}\right)$

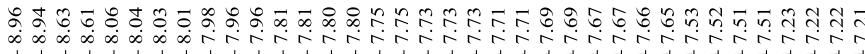

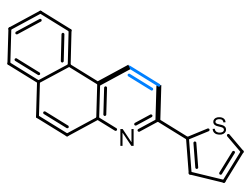

3!

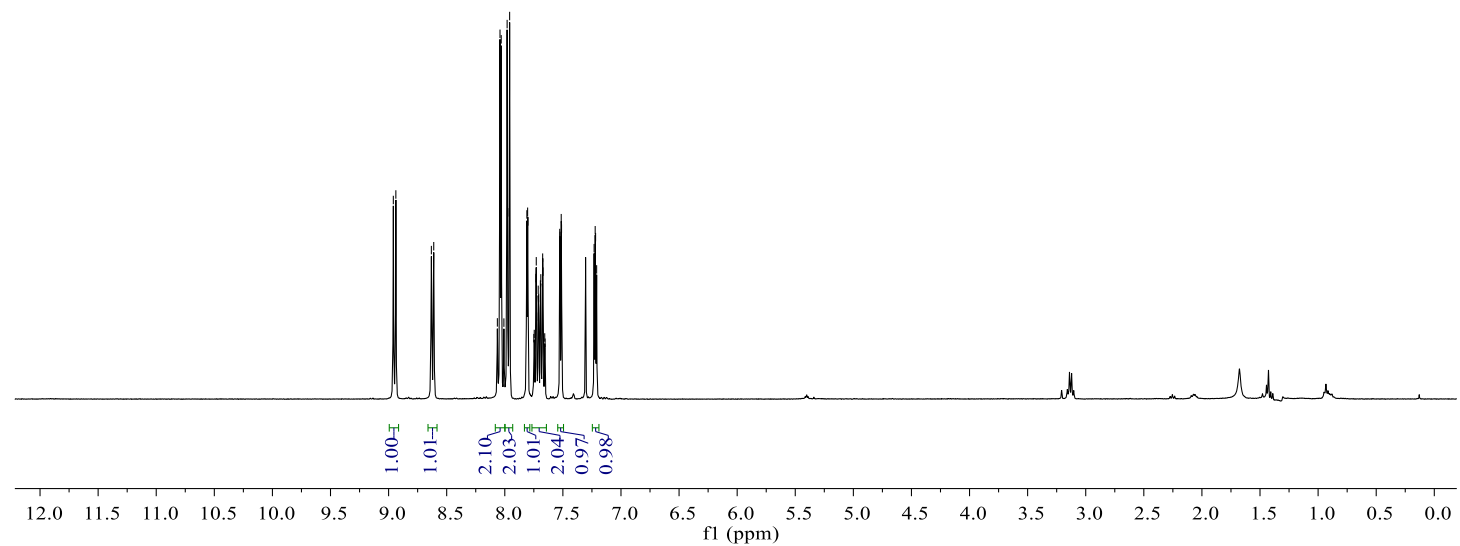


${ }^{13} \mathrm{C} \mathrm{NMR}$ of 31 (100 MHz, $\left.\mathrm{CDCl}_{3}\right)$

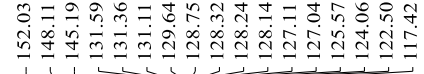

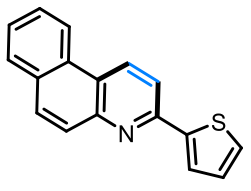

3!

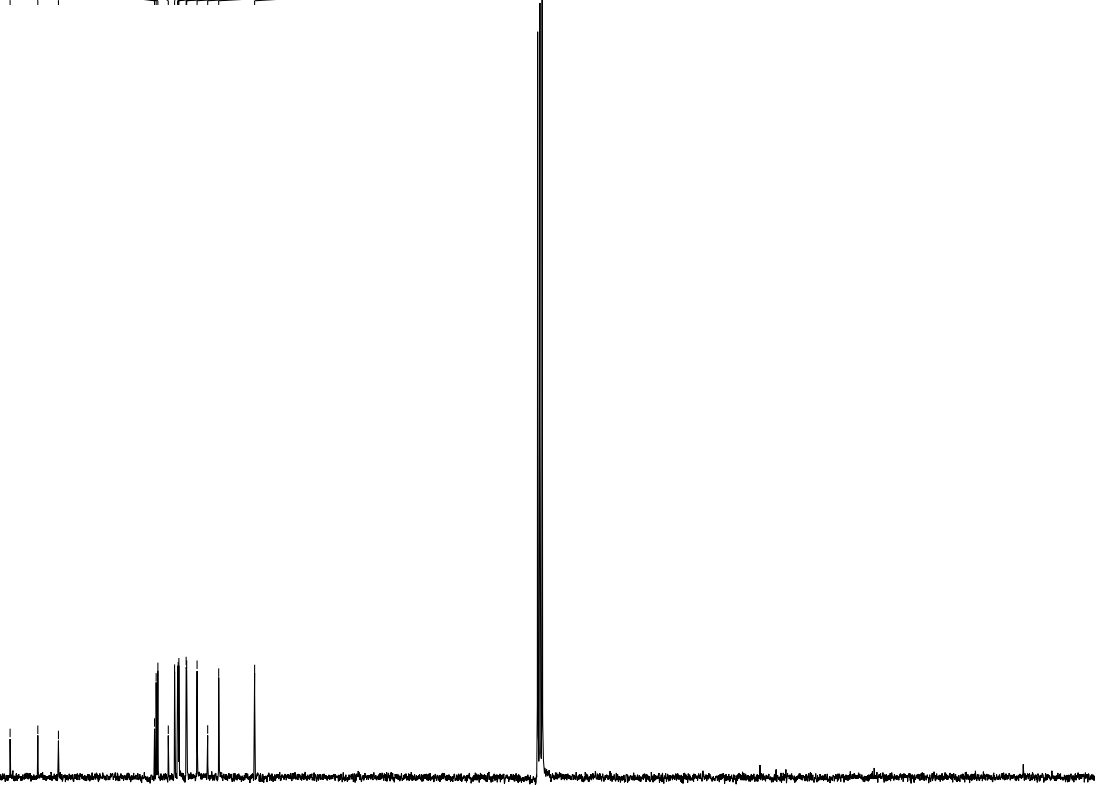

${ }^{1} \mathrm{H}$ NMR of $\mathbf{3 m}\left(400 \mathrm{MHz}, \mathrm{CDCl}_{3}\right)$

$\bigcup^{10}$<smiles>COc1ccc2ccc3nc(-c4ccccc4)ccc3c2c1</smiles>

$3 m$

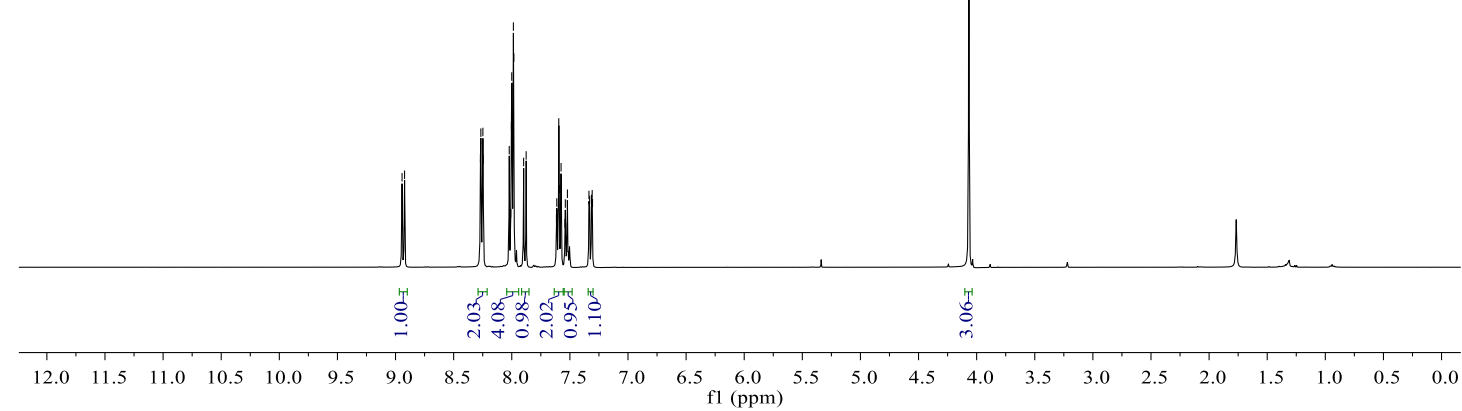


${ }^{13} \mathrm{C} \mathrm{NMR}$ of $3 \mathbf{m}\left(100 \mathrm{MHz}, \mathrm{CDCl}_{3}\right)$

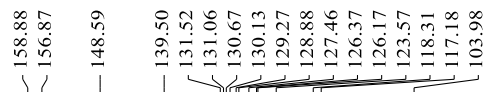<smiles>COc1ccc2ccc3nc(-c4ccccc4)ccc3c2c1</smiles>

$3 m$

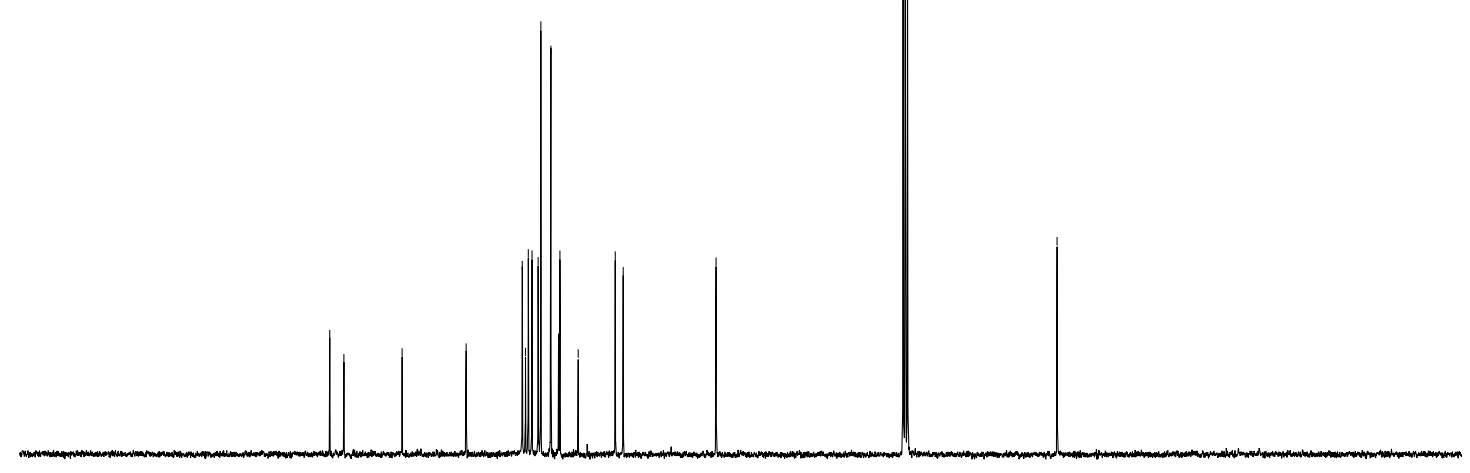

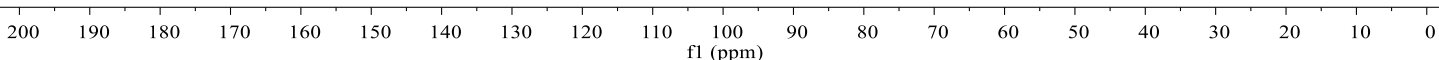

${ }^{1} \mathrm{H}$ NMR of 3n $\left(400 \mathrm{MHz}, \mathrm{CDCl}_{3}\right)$

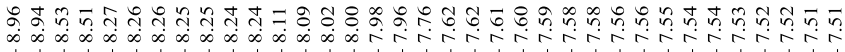

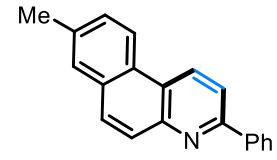

$3 n$

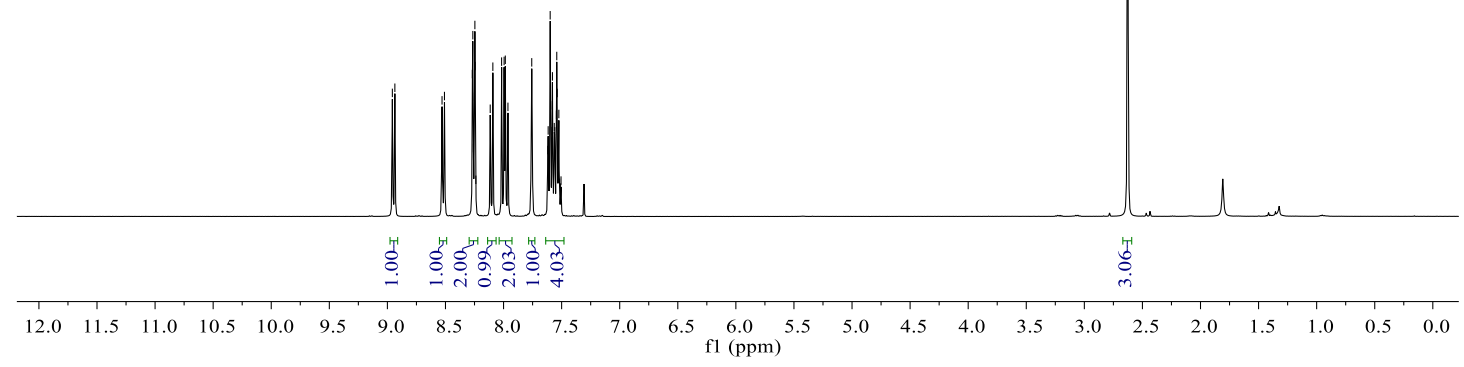


${ }^{13} \mathrm{C}$ NMR of 3n $\left(100 \mathrm{MHz}, \mathrm{CDCl}_{3}\right)$

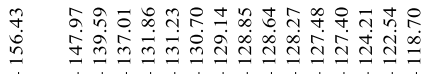

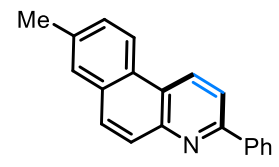

3n

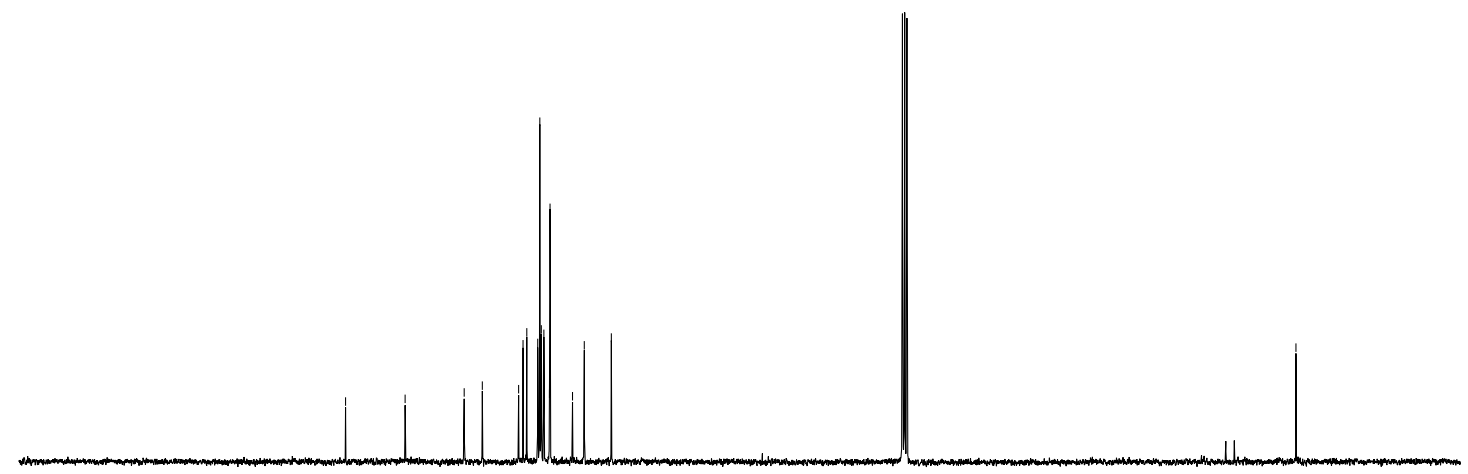

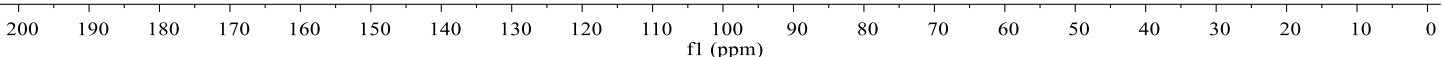

${ }^{1} \mathrm{H}$ NMR of $3 \mathbf{o}\left(400 \mathrm{MHz}, \mathrm{CDCl}_{3}\right)$

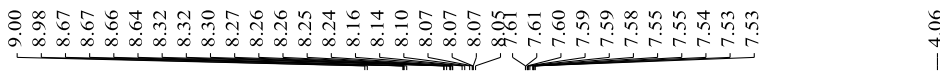

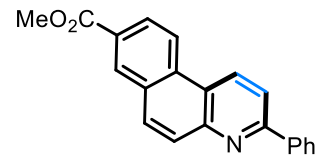

30

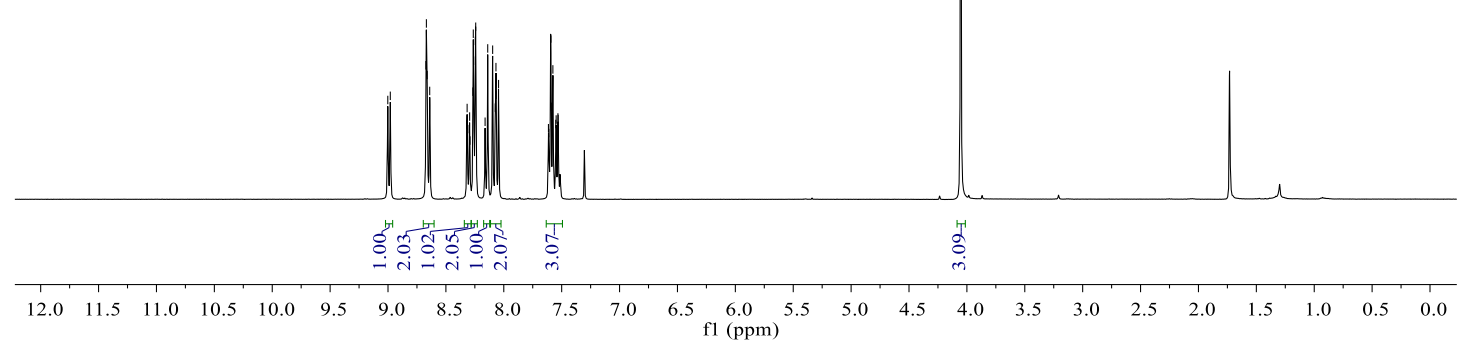


${ }^{13} \mathrm{C}$ NMR of $30\left(100 \mathrm{MHz}, \mathrm{CDCl}_{3}\right)$

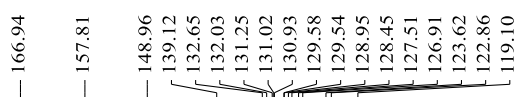

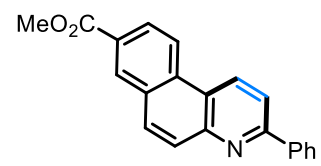

30

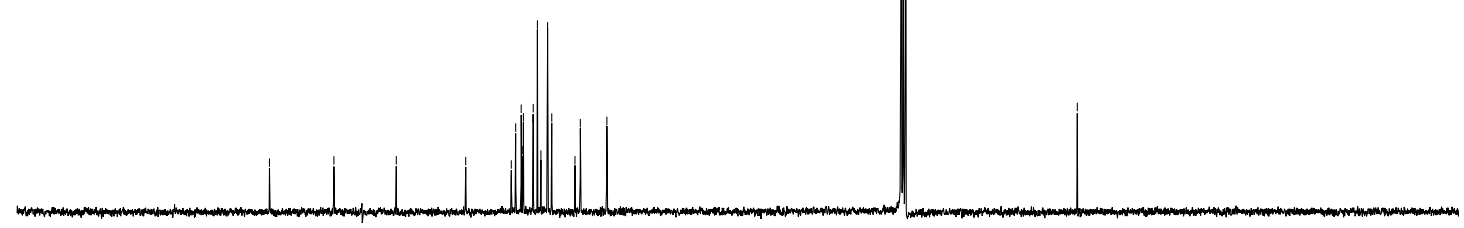

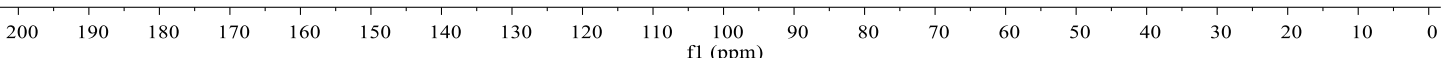

${ }^{1} \mathrm{H}$ NMR of 3p $\left(400 \mathrm{MHz}, \mathrm{CDCl}_{3}\right)$

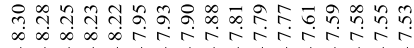

$\overbrace{\mathrm{N}}$

$3 p$

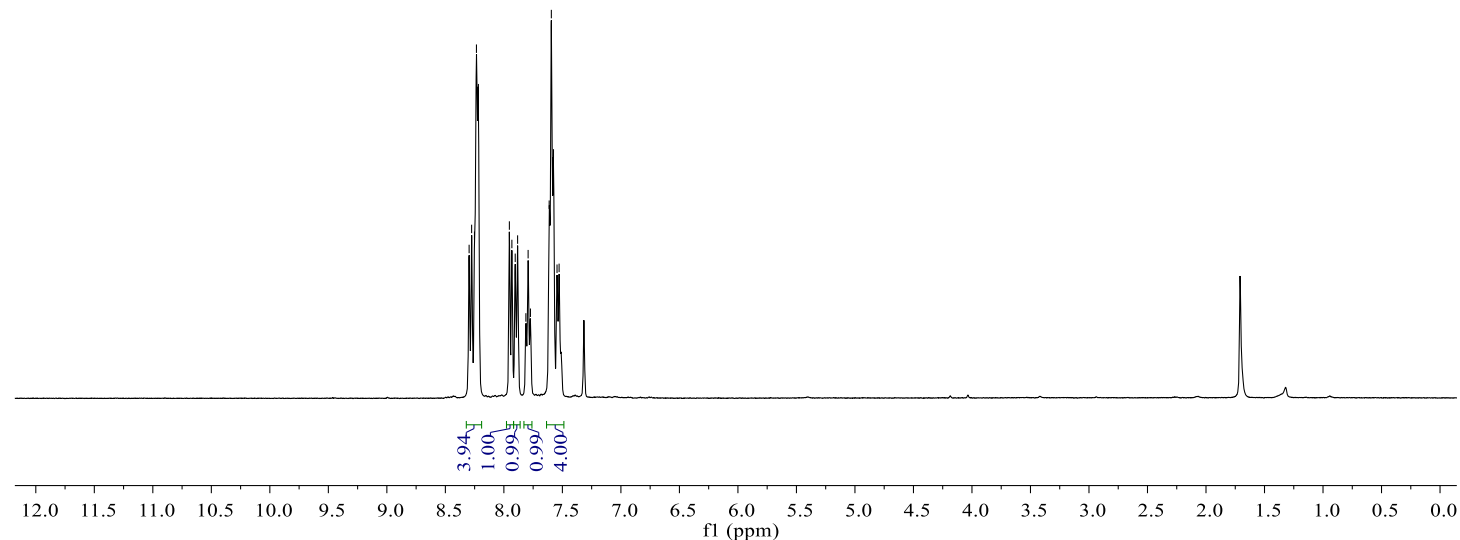


${ }^{13} \mathrm{C}$ NMR of $\mathbf{3 p}\left(100 \mathrm{MHz}, \mathrm{CDCl}_{3}\right)$

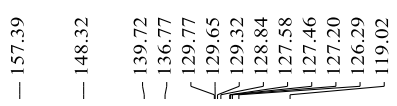

$\mathrm{Did}_{\mathrm{ph}}$

$3 p$

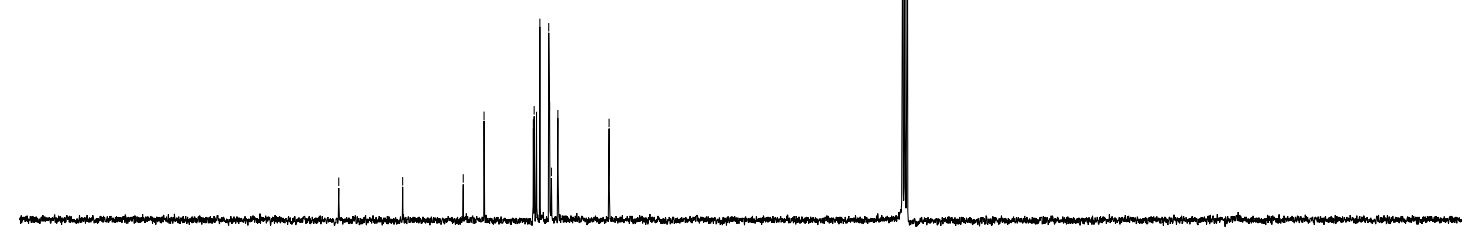

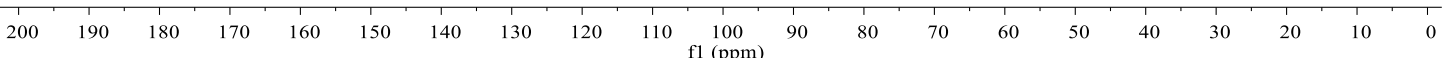

${ }^{1} \mathrm{H} \mathrm{NMR}$ of $\mathbf{3 q}\left(400 \mathrm{MHz}, \mathrm{CDCl}_{3}\right)$

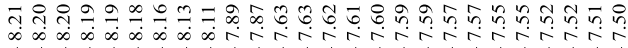

Melloh

3q

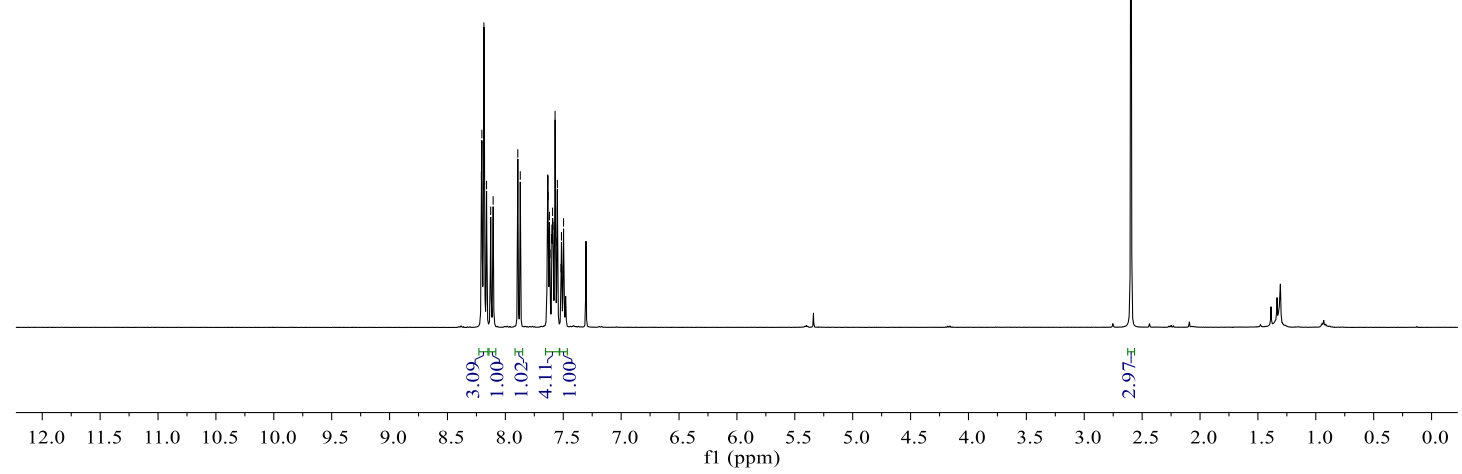


${ }^{13} \mathrm{C}$ NMR of 3q $\left(100 \mathrm{MHz}, \mathrm{CDCl}_{3}\right)$

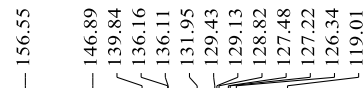

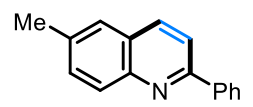

$3 q$

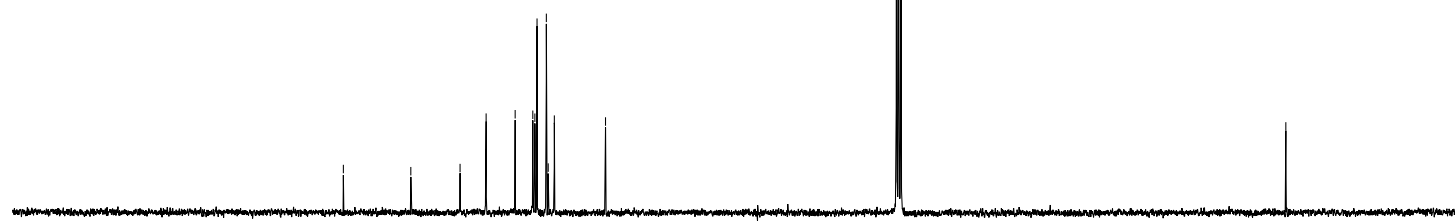

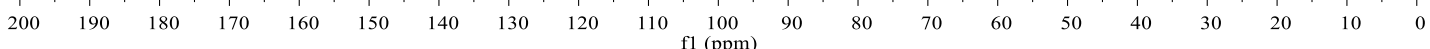

${ }^{1} \mathrm{H}$ NMR of $3 \mathbf{r}\left(400 \mathrm{MHz}, \mathrm{CDCl}_{3}\right)$

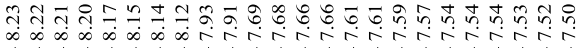

$\mathrm{MeS}_{\mathrm{Ph}}^{\mathrm{MeS}}$

$3 r$

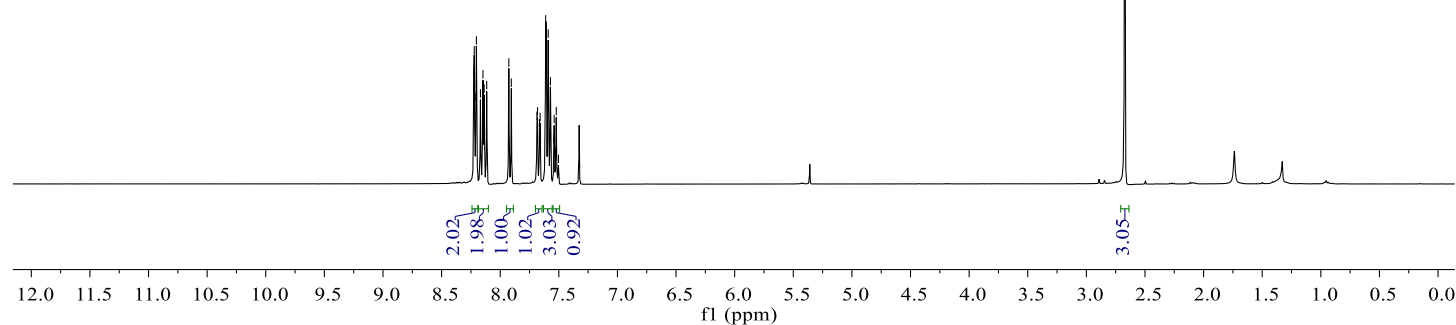


${ }^{13} \mathrm{C}$ NMR of $3 \mathbf{r}\left(100 \mathrm{MHz}, \mathrm{CDCl}_{3}\right)$

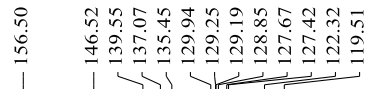

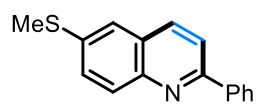

$3 \mathbf{r}$

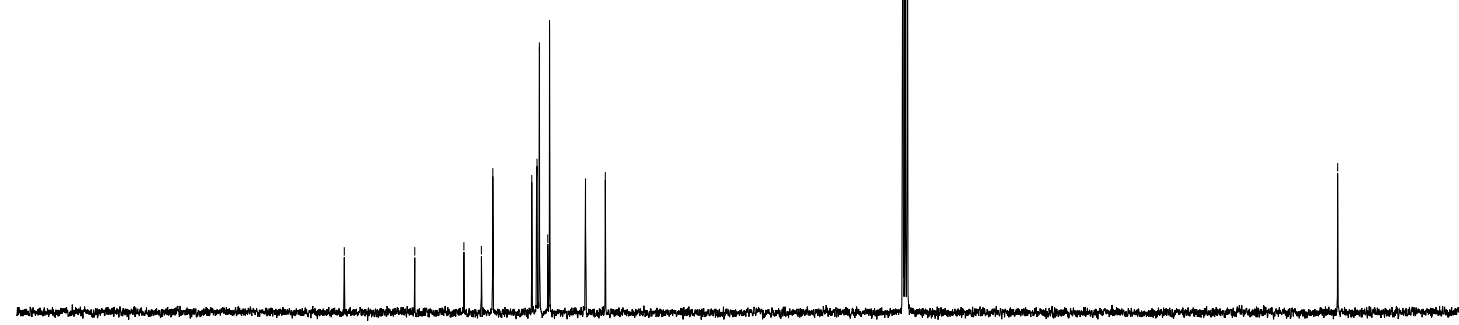

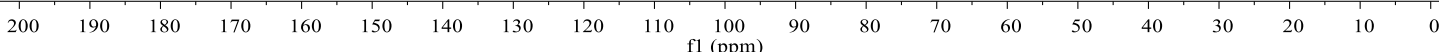

${ }^{1} \mathrm{H}$ NMR of $3 s\left(400 \mathrm{MHz}, \mathrm{CDCl}_{3}\right)$

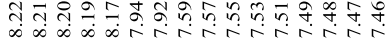

$\mathrm{P}_{\mathrm{Nh}}^{\mathrm{C}}$

$3 \mathbf{s}$

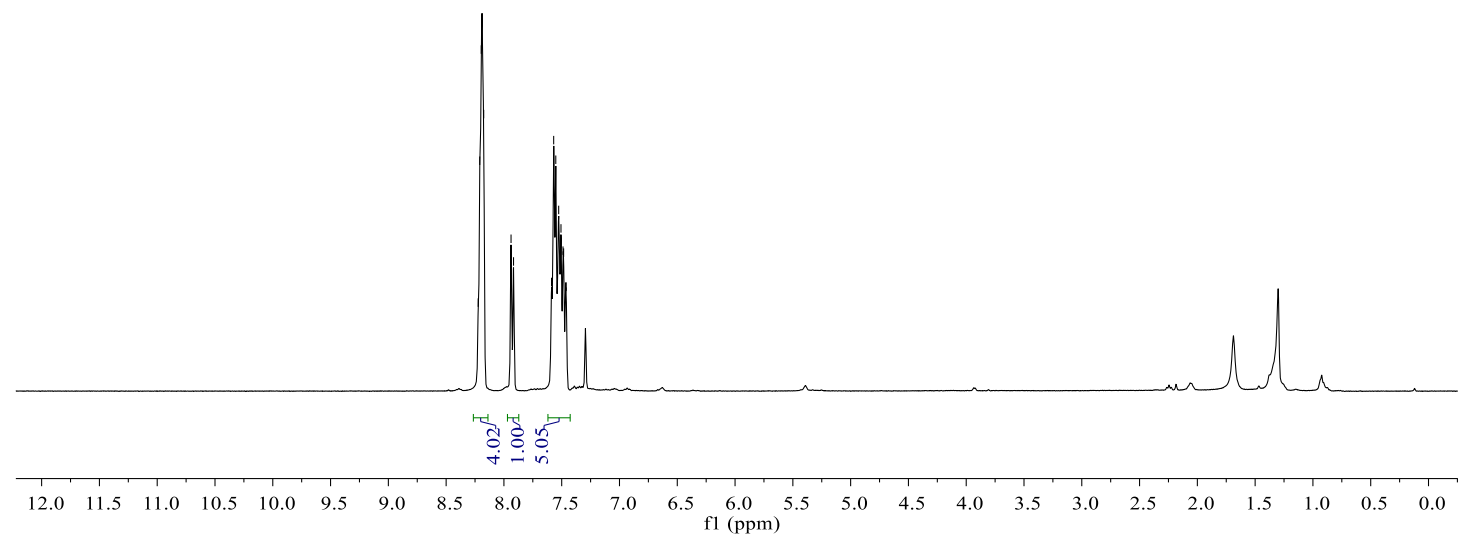


${ }^{13} \mathrm{C}$ NMR of $3 \mathbf{s}\left(100 \mathrm{MHz}, \mathrm{CDCl}_{3}\right)$

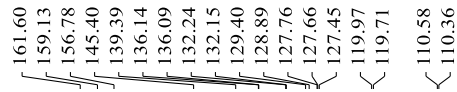

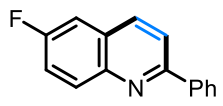

3s

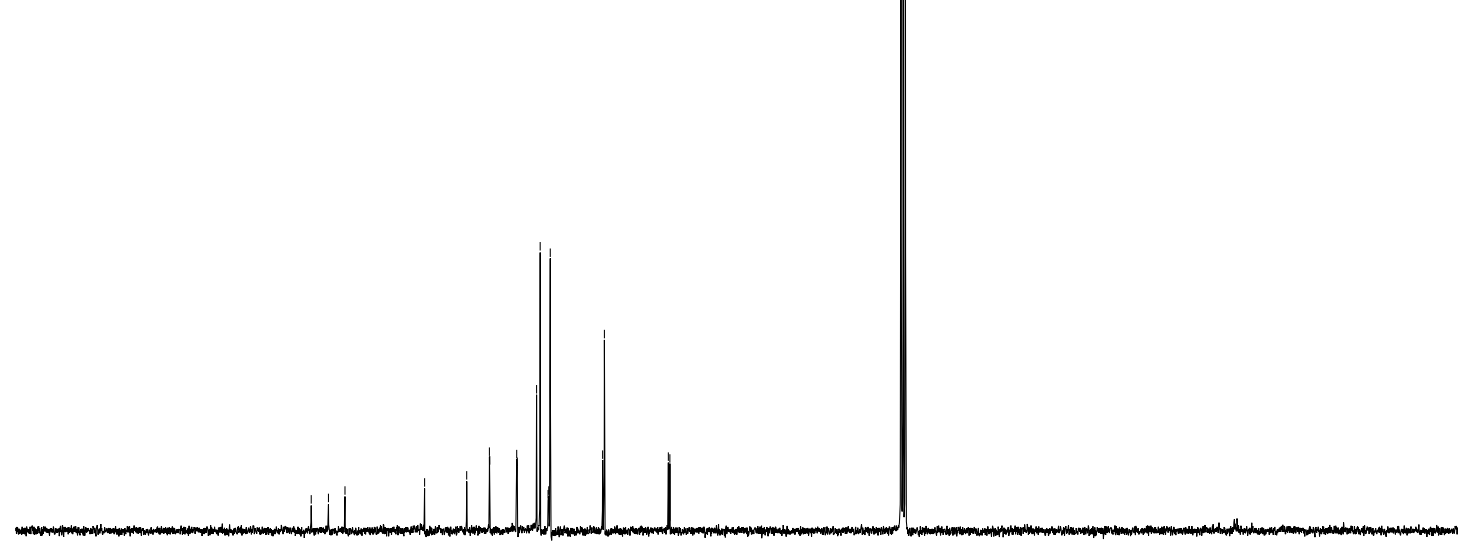

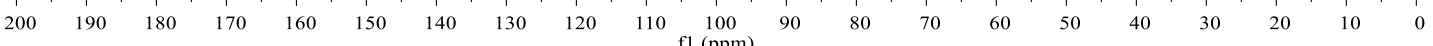

${ }^{19} \mathrm{~F}$ NMR of $3 \mathbf{s}\left(376 \mathrm{MHz}, \mathrm{CDCl}_{3}\right)$<smiles>Fc1ccc2nc(-c3ccccc3)ccc2c1</smiles>

$3 \mathbf{s}$

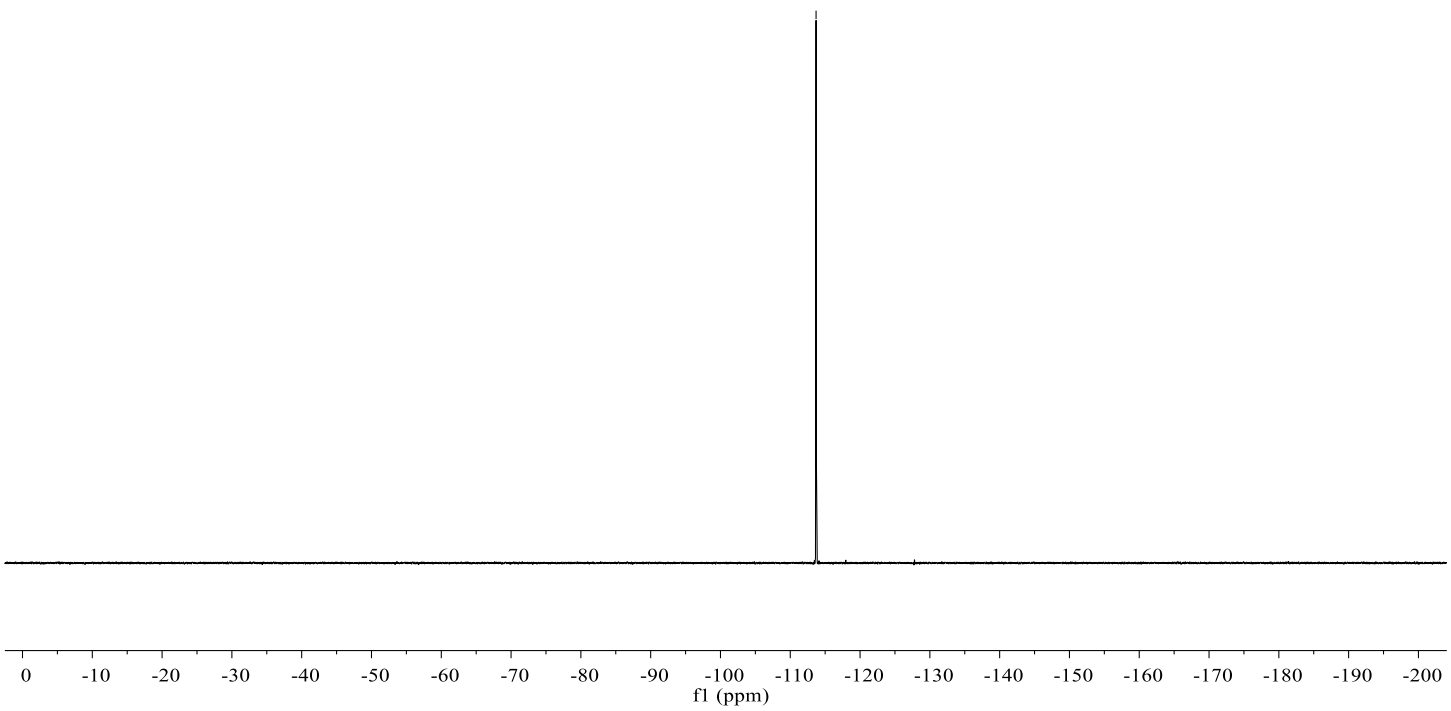


${ }^{1} \mathrm{H}$ NMR of $3 \mathbf{t}\left(400 \mathrm{MHz}, \mathrm{CDCl}_{3}\right)$

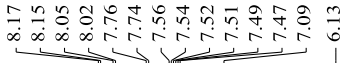

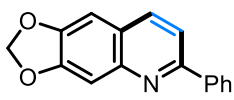

$3 t$

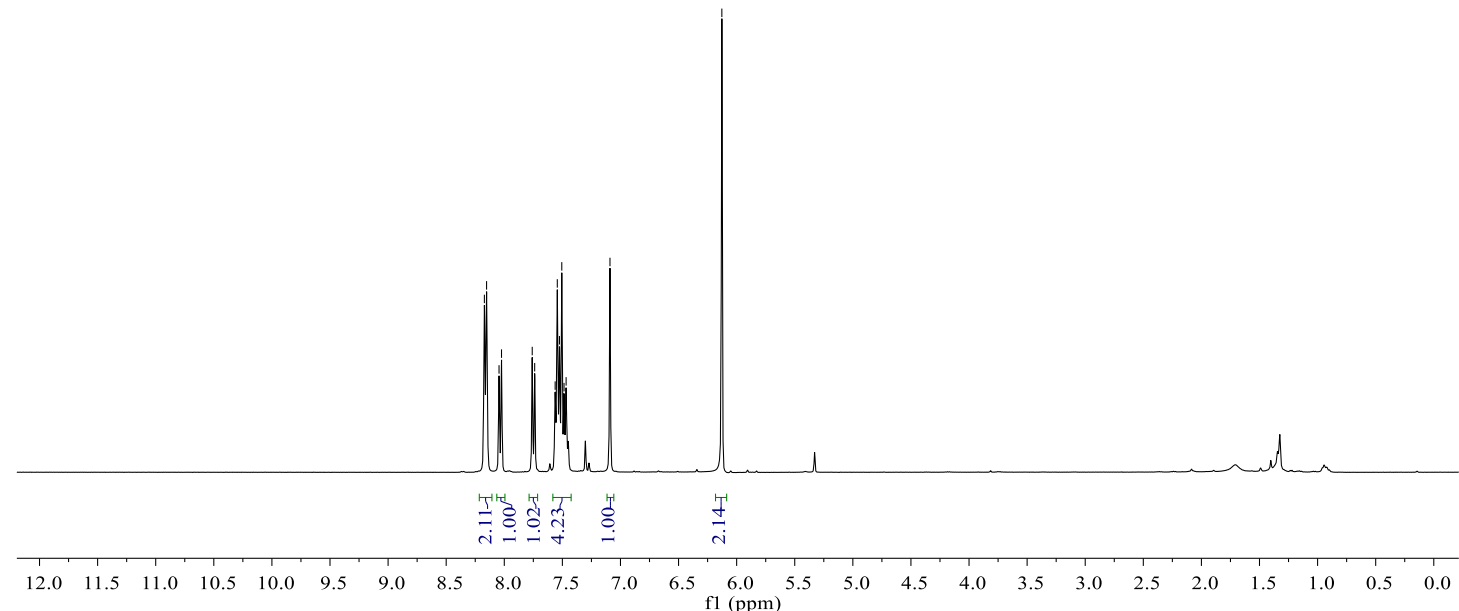

${ }^{13} \mathrm{C}$ NMR of $3 \mathbf{t}\left(100 \mathrm{MHz}, \mathrm{CDCl}_{3}\right)$

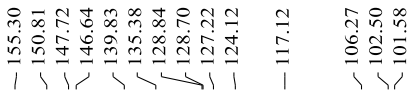

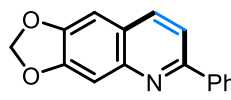

$3 t$
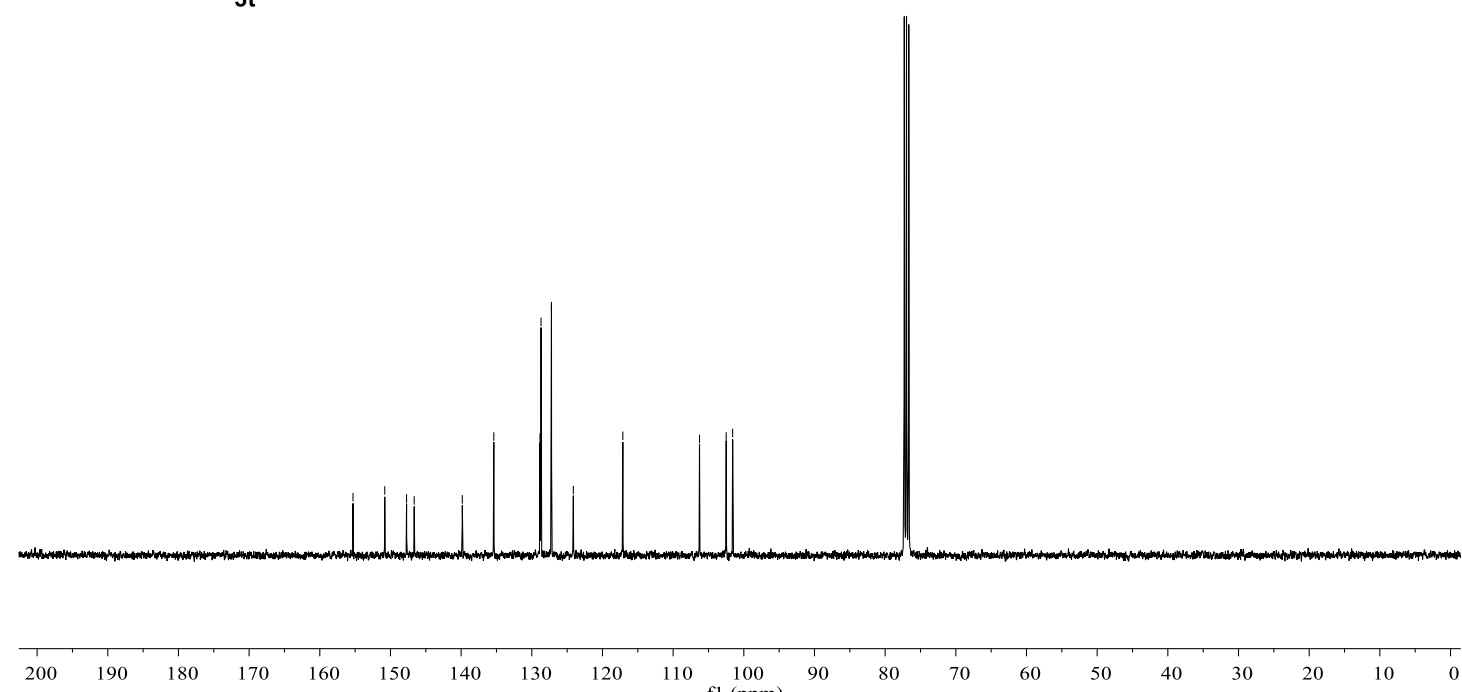
${ }^{1} \mathrm{H}$ NMR of $3 \mathbf{u}\left(400 \mathrm{MHz}, \mathrm{CDCl}_{3}\right)$

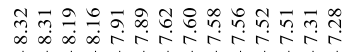

trat

$\overbrace{3 u}$

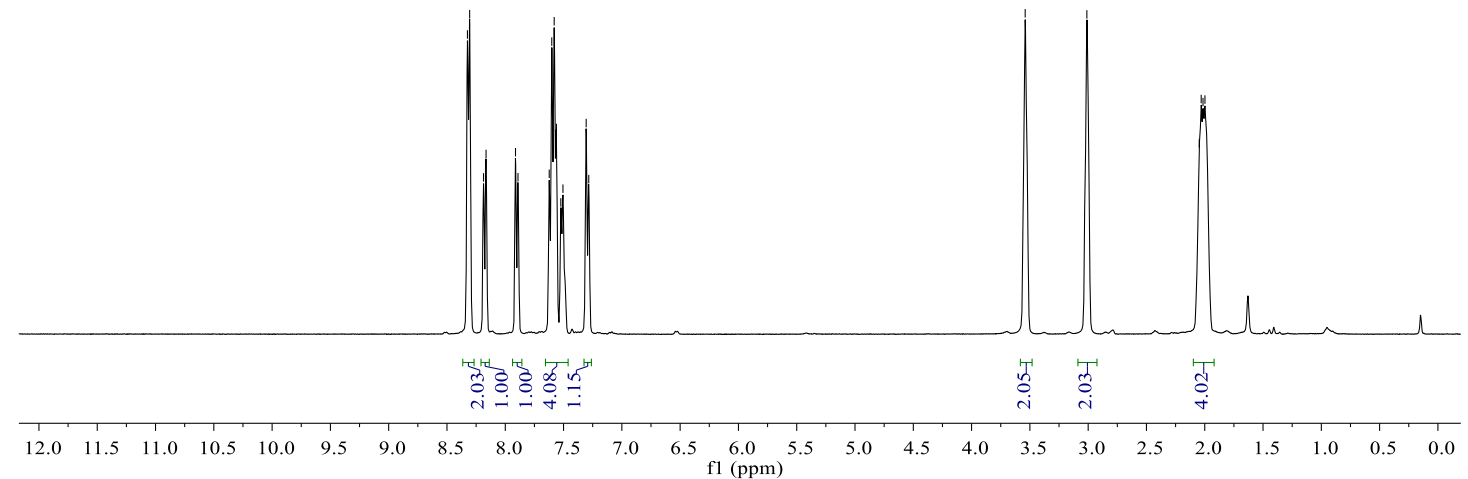

${ }^{13} \mathrm{C}$ NMR of $3 \mathbf{u}\left(100 \mathrm{MHz}, \mathrm{CDCl}_{3}\right)$

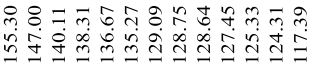

$\infty$

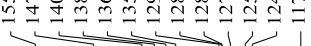

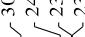

$\overbrace{3 u}$

|

$\begin{array}{llllllllllllllllllllll}200 & 190 & 180 & 170 & 160 & 150 & 140 & 130 & 120 & 110 & 100 & 90 & 80 & 70 & 60 & 50 & 40 & 30 & 20 & 10 & 0\end{array}$ 
${ }^{1} \mathrm{H}$ NMR of 3a' $\left(400 \mathrm{MHz}, \mathrm{CDCl}_{3}\right)$

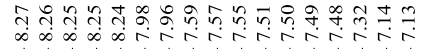

$\overbrace{\mathrm{OMe}} \mathrm{Cl}_{\mathrm{Ph}}$

$3 a^{\prime}$

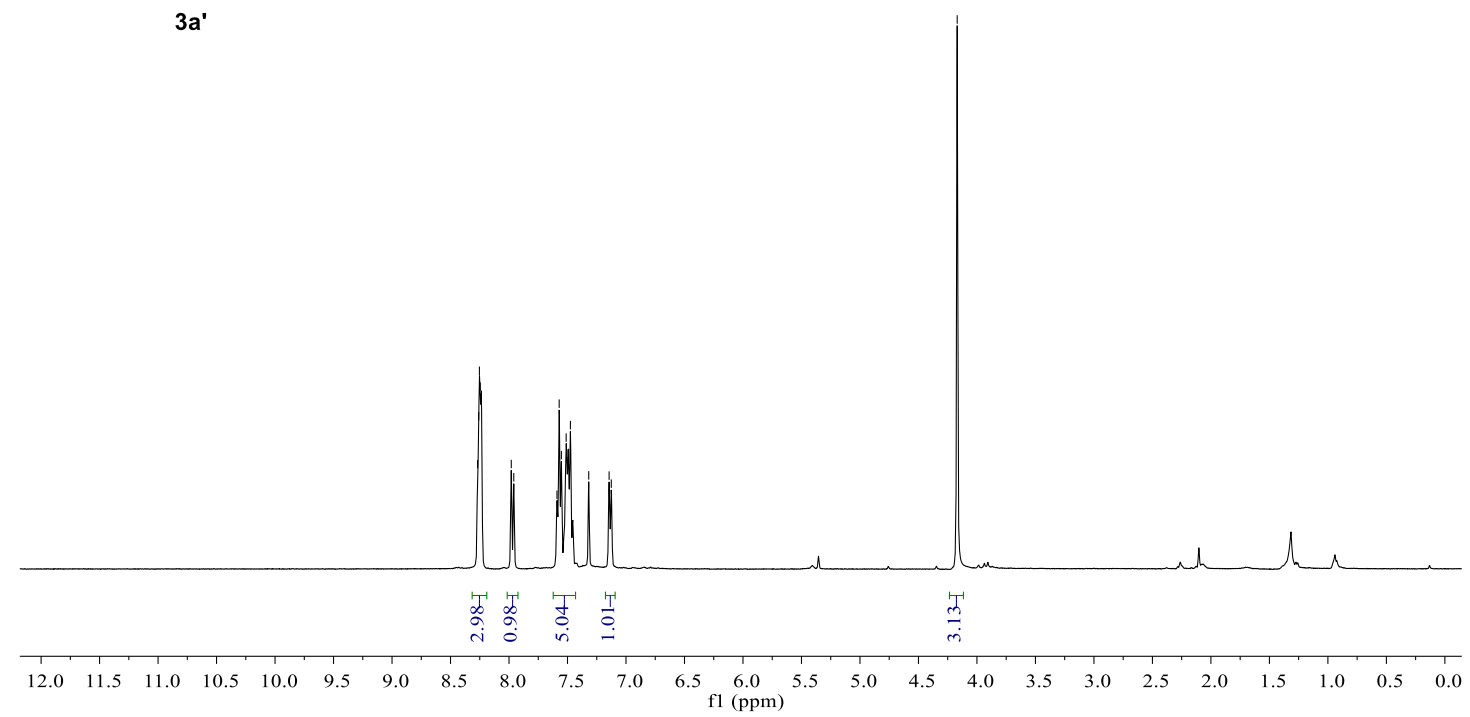

${ }^{13} \mathrm{C}$ NMR of 3a' (100 MHz, $\left.\mathrm{CDCl}_{3}\right)$

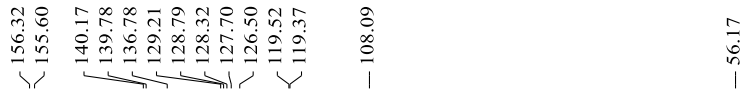

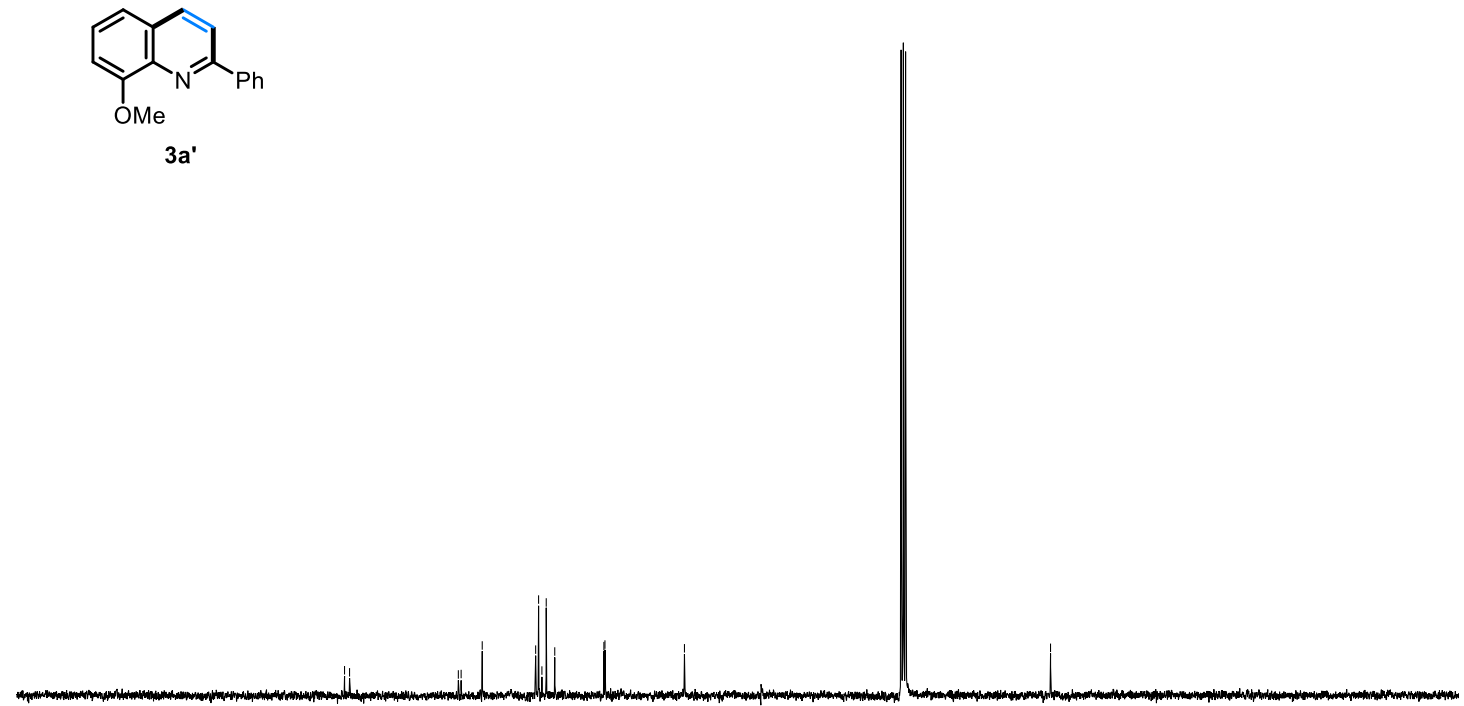

3a'

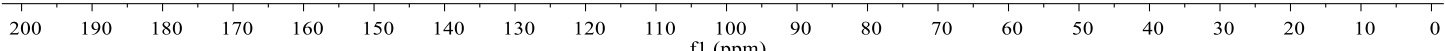


${ }^{1} \mathrm{H}$ NMR of 3b' (400 MHz, $\mathrm{CDCl}_{3}$ )

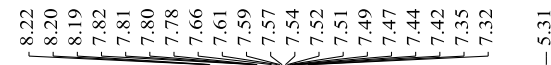

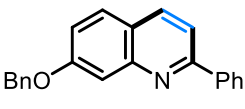

3b'

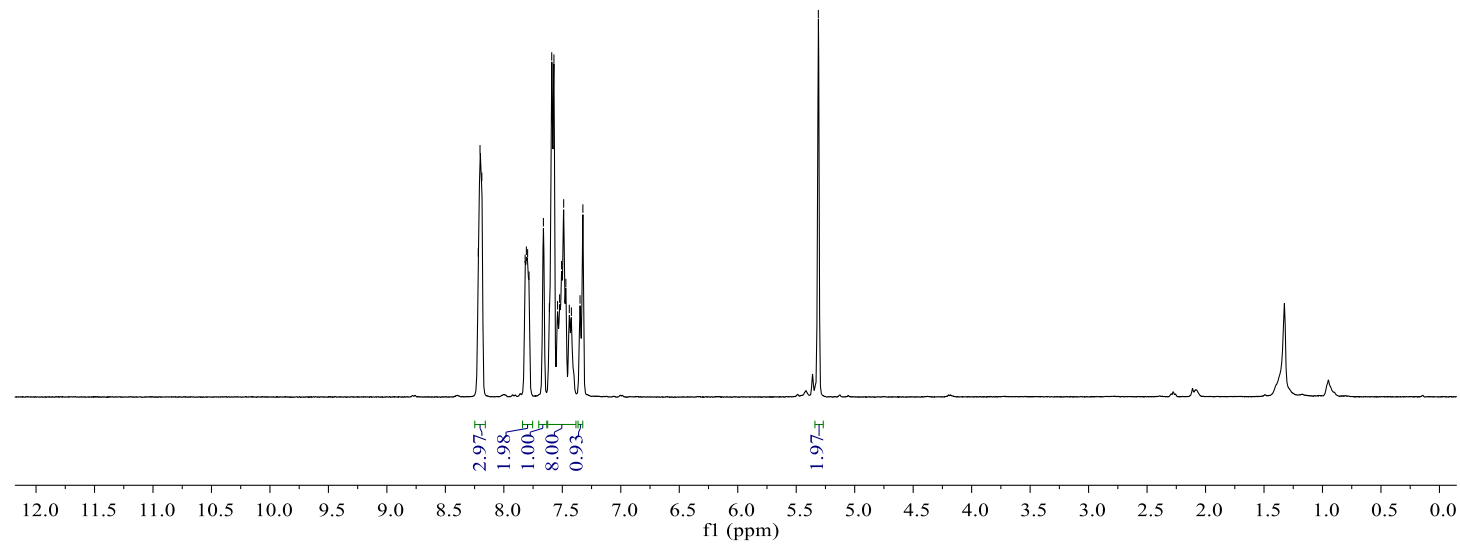

${ }^{13} \mathrm{C}$ NMR of 3b' $\left(100 \mathrm{MHz}, \mathrm{CDCl}_{3}\right)$

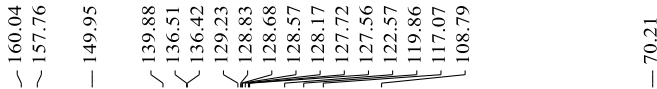

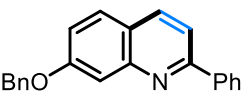

3b'
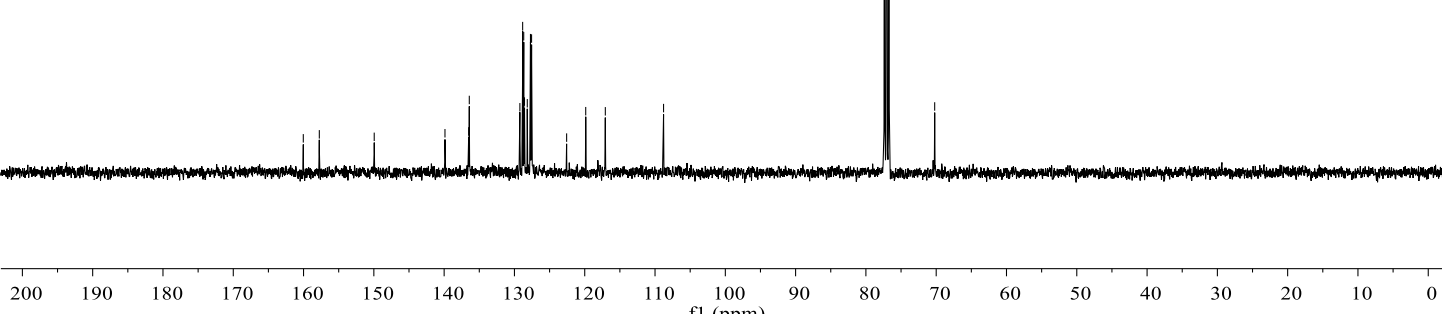
${ }^{1} \mathrm{H}$ NMR of $3 \mathbf{c}^{\prime}\left(400 \mathrm{MHz}, \mathrm{CDCl}_{3}\right)$

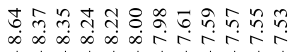

$\mathrm{EtO}_{2} \mathrm{C}$<smiles>c1ccc2nc(-c3cccc4ccccc34)ccc2c1</smiles>

$3 c^{\prime}$

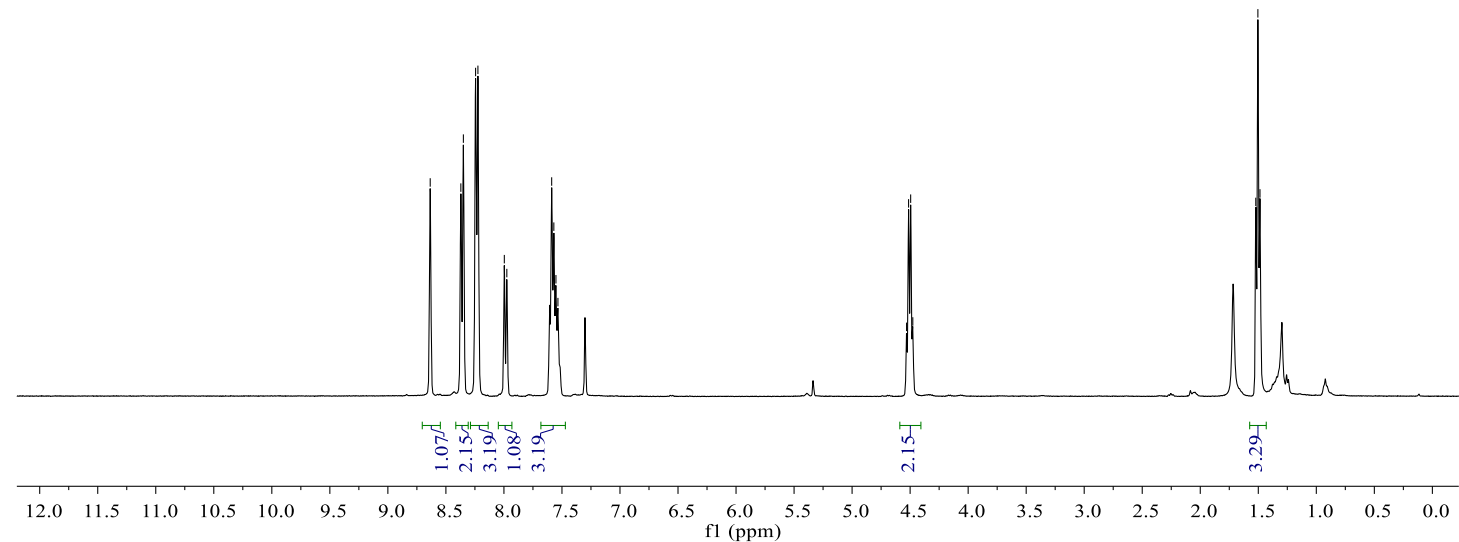

${ }^{13} \mathrm{C}$ NMR of $\mathbf{3 c}$ (100 MHz, $\left.\mathrm{CDCl}_{3}\right)$

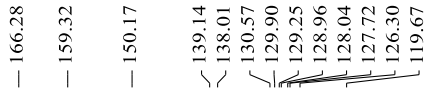
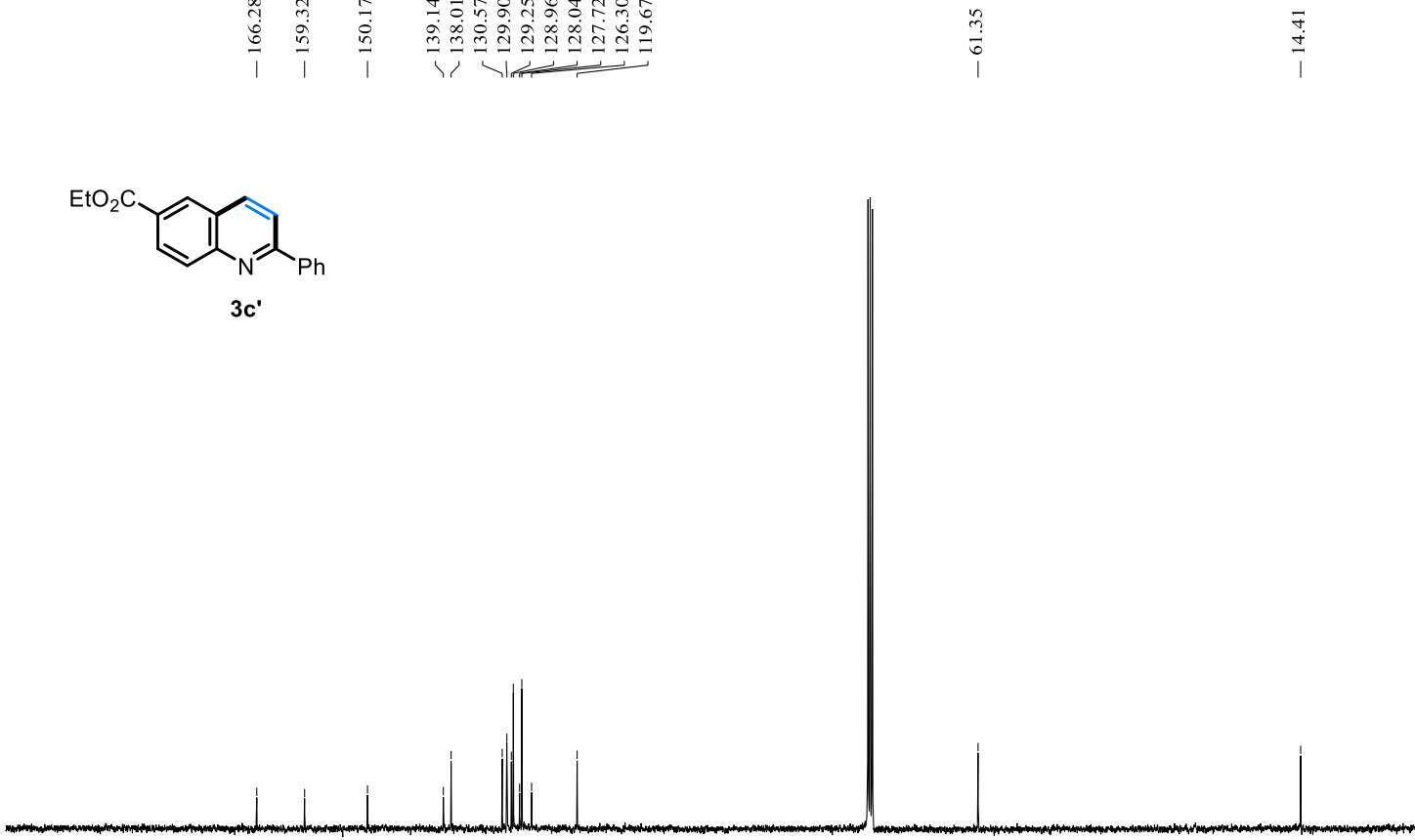
${ }^{1} \mathrm{H}$ NMR of 3d' (400 MHz, $\mathrm{CDCl}_{3}$ )

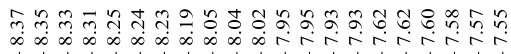

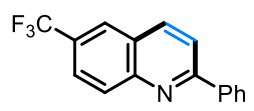

3d'

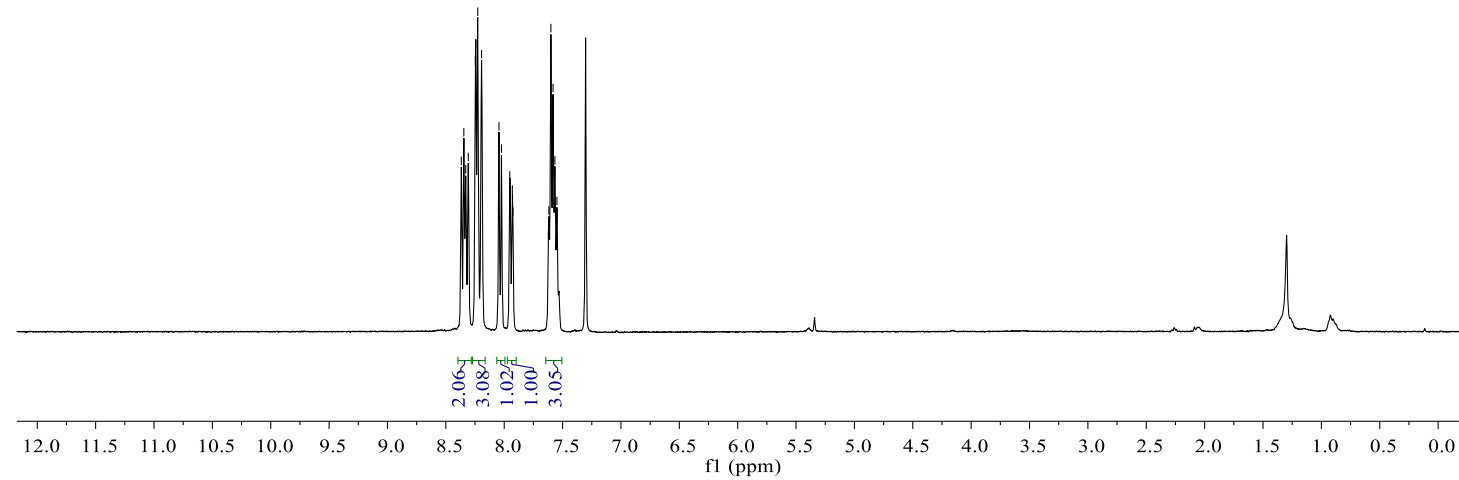

${ }^{13} \mathrm{C}$ NMR of 3d' (100 MHz, $\left.\mathrm{CDCl}_{3}\right)$

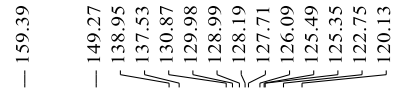

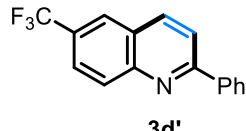

3d'

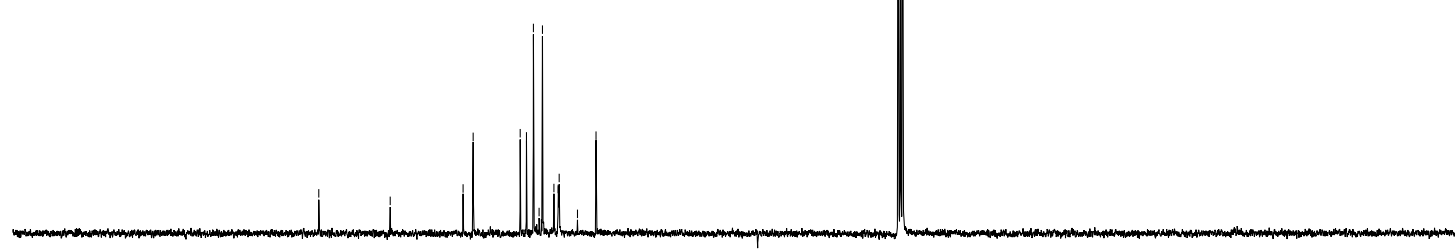

$\begin{array}{lllllllllllllllllllll}200 & 190 & 180 & 170 & 160 & 150 & 140 & 130 & 120 & 110 & 100 & 90 & 80 & 70 & 60 & 50 & 40 & 30 & 20 & 10 & 0\end{array}$ 
${ }^{19} \mathrm{~F}$ NMR of 3d' (376 MHz, $\mathrm{CDCl}_{3}$ )

$\infty$
$\stackrel{c}{i}$
$i$
1
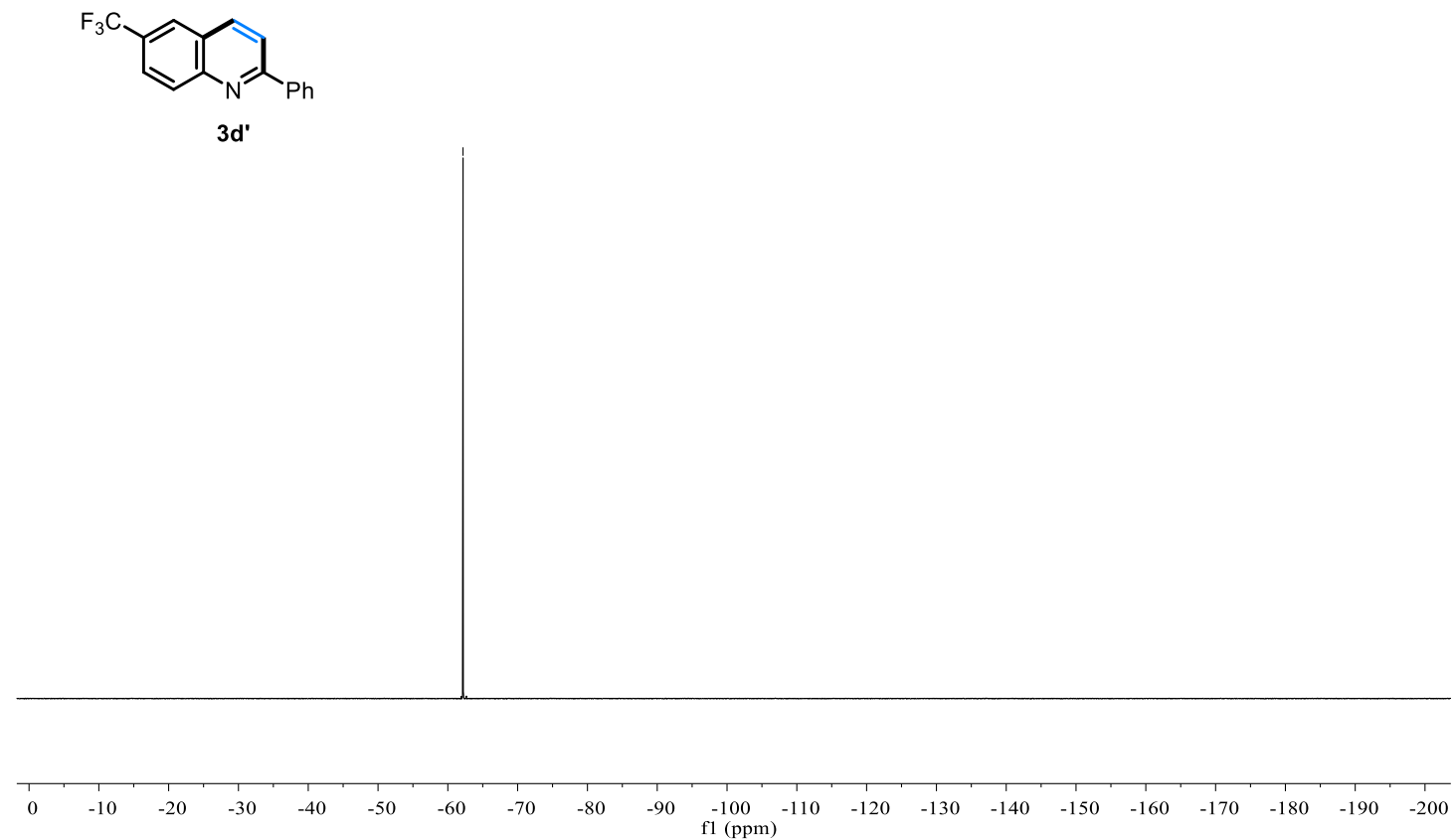

${ }^{1} \mathrm{H}$ NMR of $3 e^{\prime}\left(400 \mathrm{MHz}, \mathrm{CDCl}_{3}\right)$

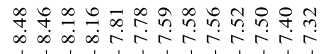

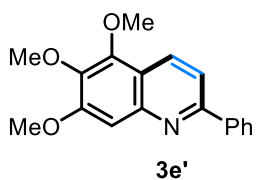

$3 e^{\prime}$

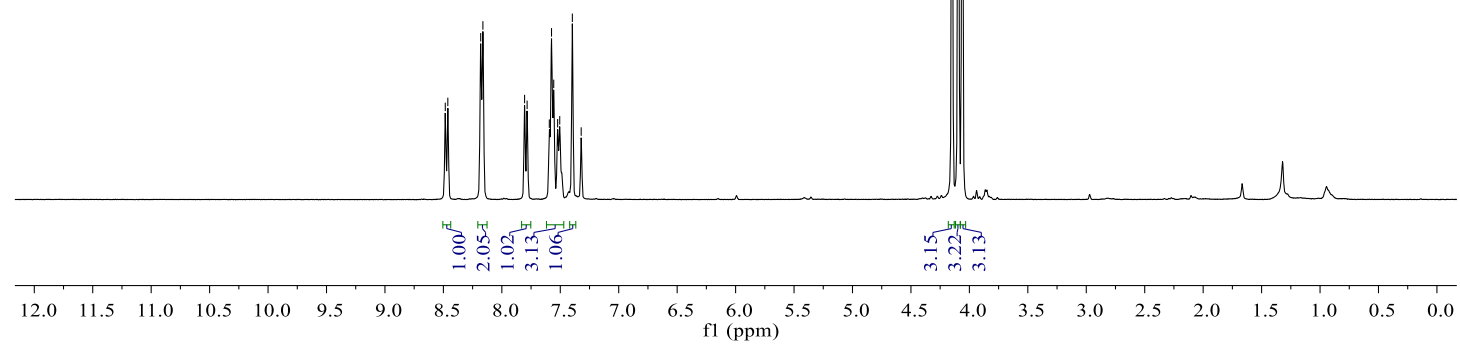


${ }^{13} \mathrm{C} \mathrm{NMR}$ of $3 \mathbf{e}^{\mathrm{\prime}}\left(100 \mathrm{MHz}, \mathrm{CDCl}_{3}\right)$

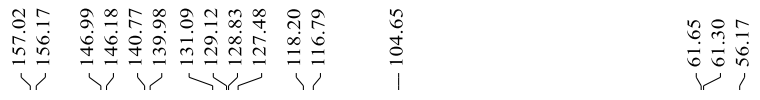

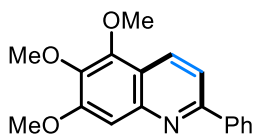

$3 e^{\prime}$

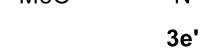


${ }^{13} \mathrm{C}$ NMR of $3 \mathbf{f f}^{\prime}\left(100 \mathrm{MHz}, \mathrm{CDCl}_{3}\right)$

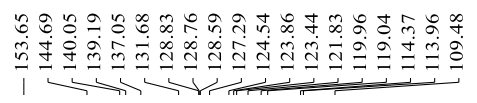

$\stackrel{\substack{n \\ i}}{i}$
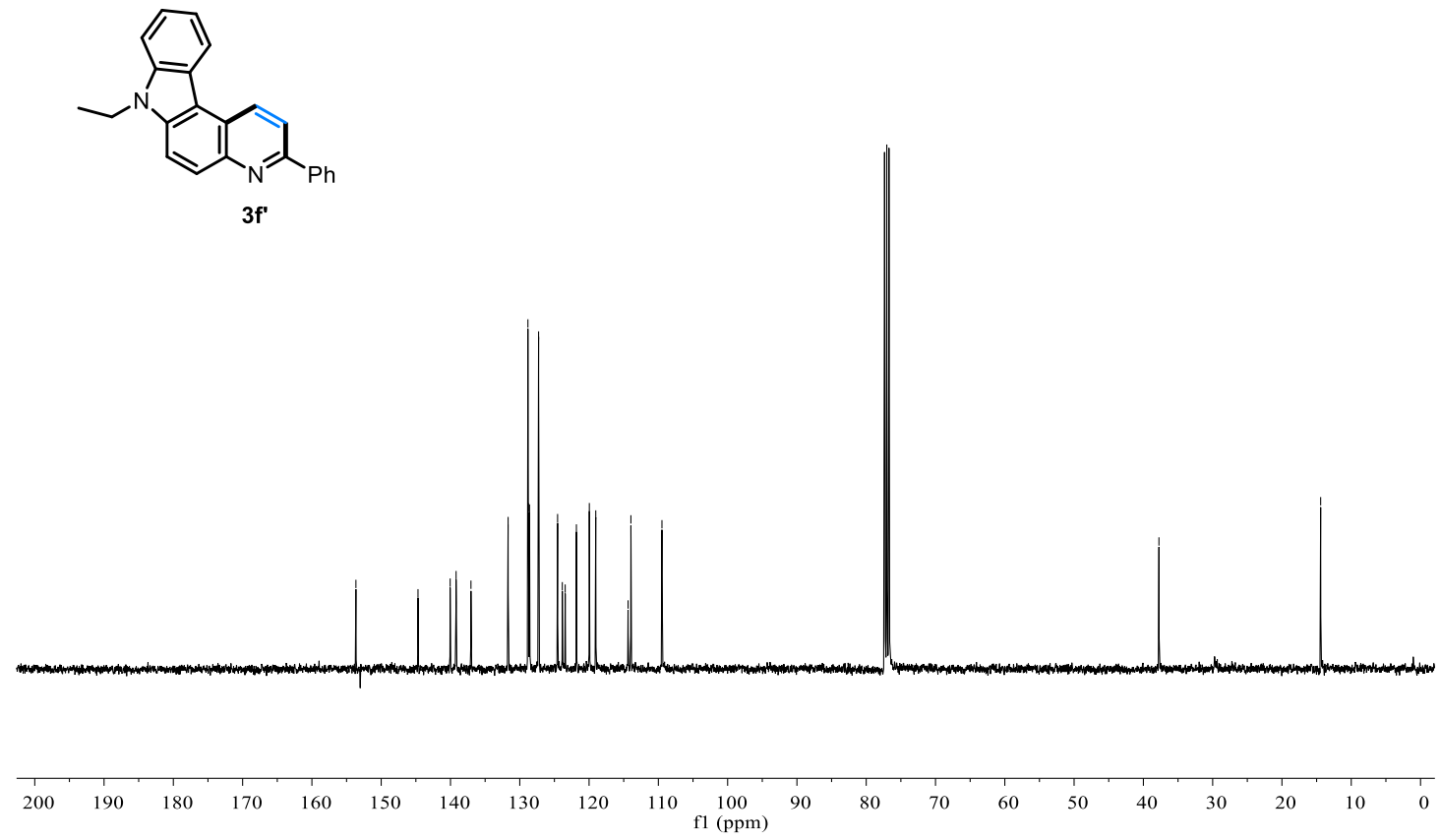

${ }^{1} \mathrm{H}$ NMR of jg' (400 MHz, DMSO)
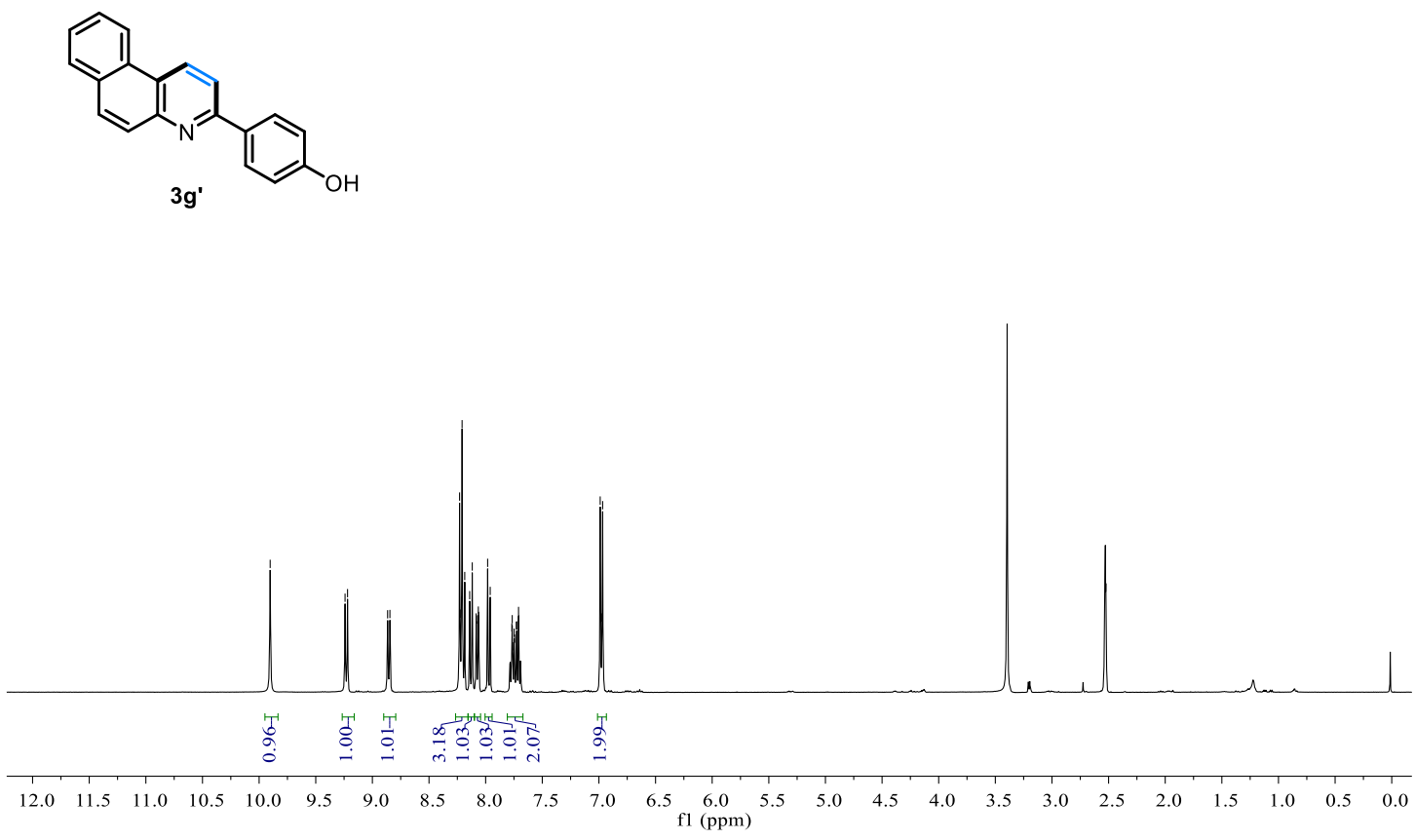

S48 
${ }^{13} \mathrm{C}$ NMR of 3g' (100 MHz, DMSO)

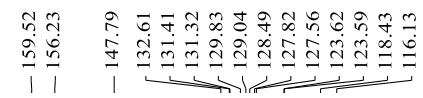
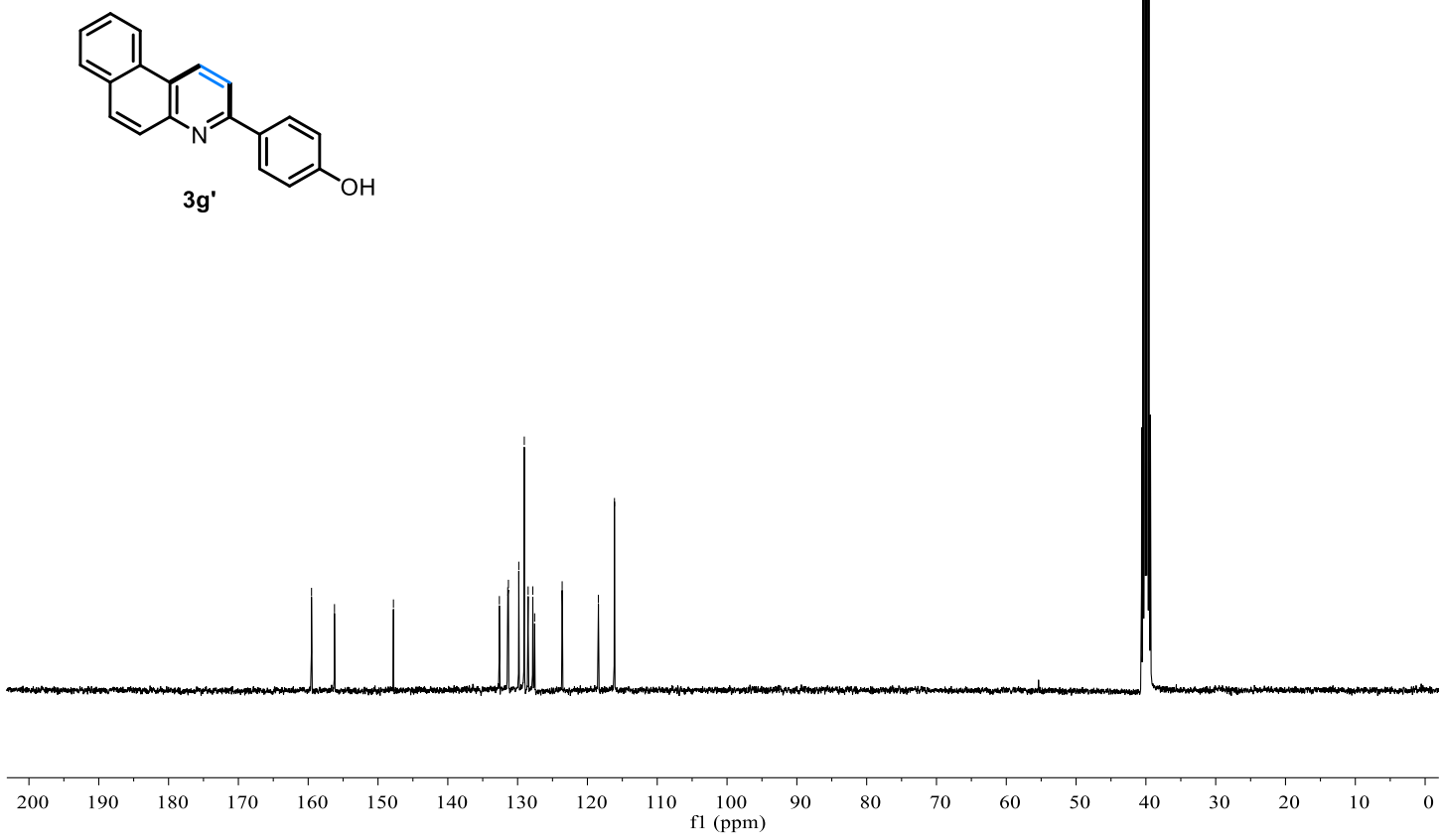

${ }^{1} \mathrm{H}$ NMR of 3h' (400 MHz, $\mathrm{CDCl}_{3}$ )

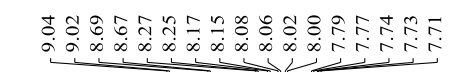
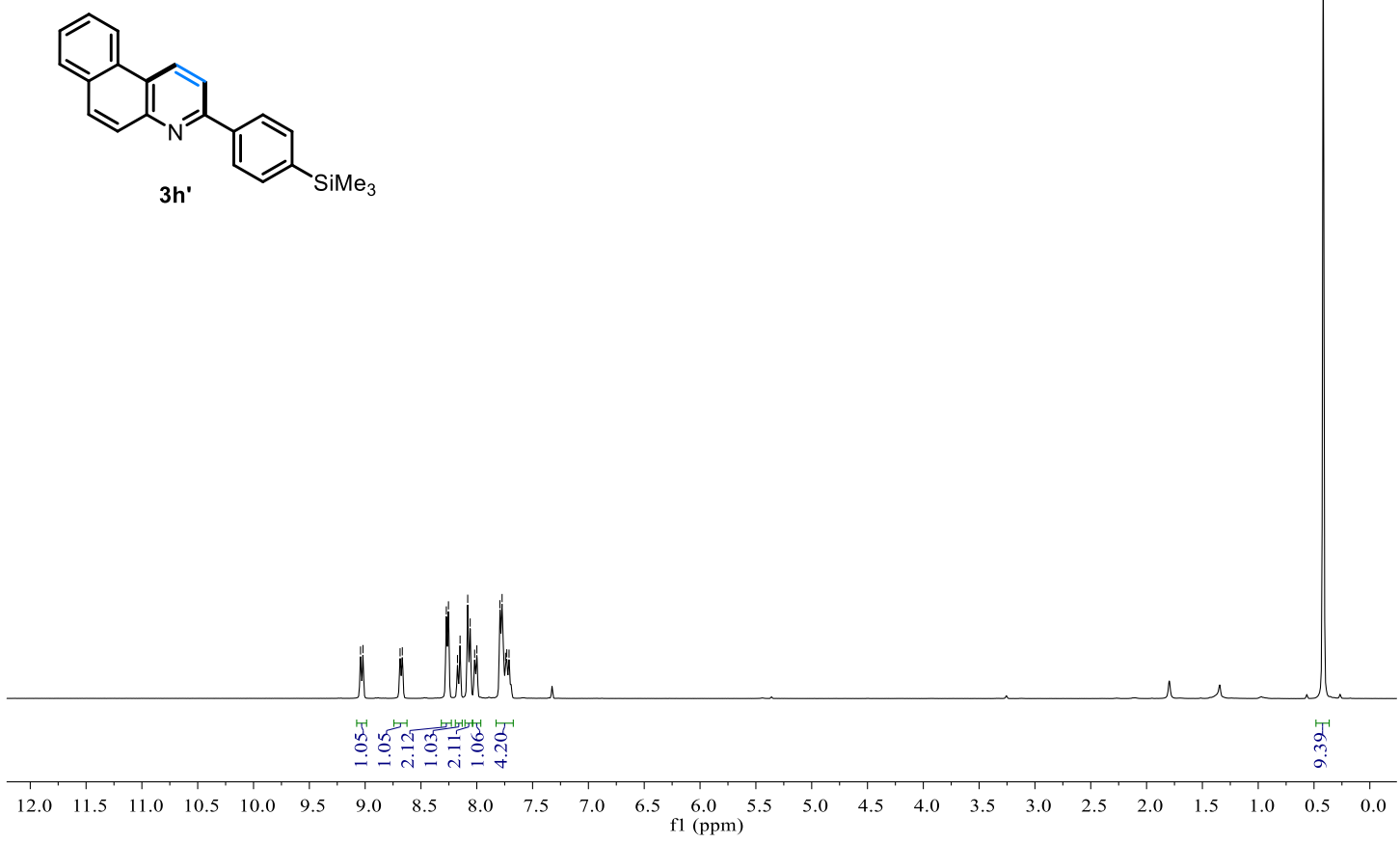

S49 
${ }^{13} \mathrm{C}$ NMR of Sh' (100 MHz, $\left.\mathrm{CDCl}_{3}\right)$

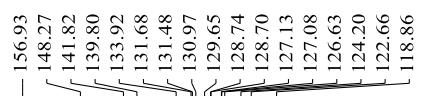

$\stackrel{\circ}{i}$
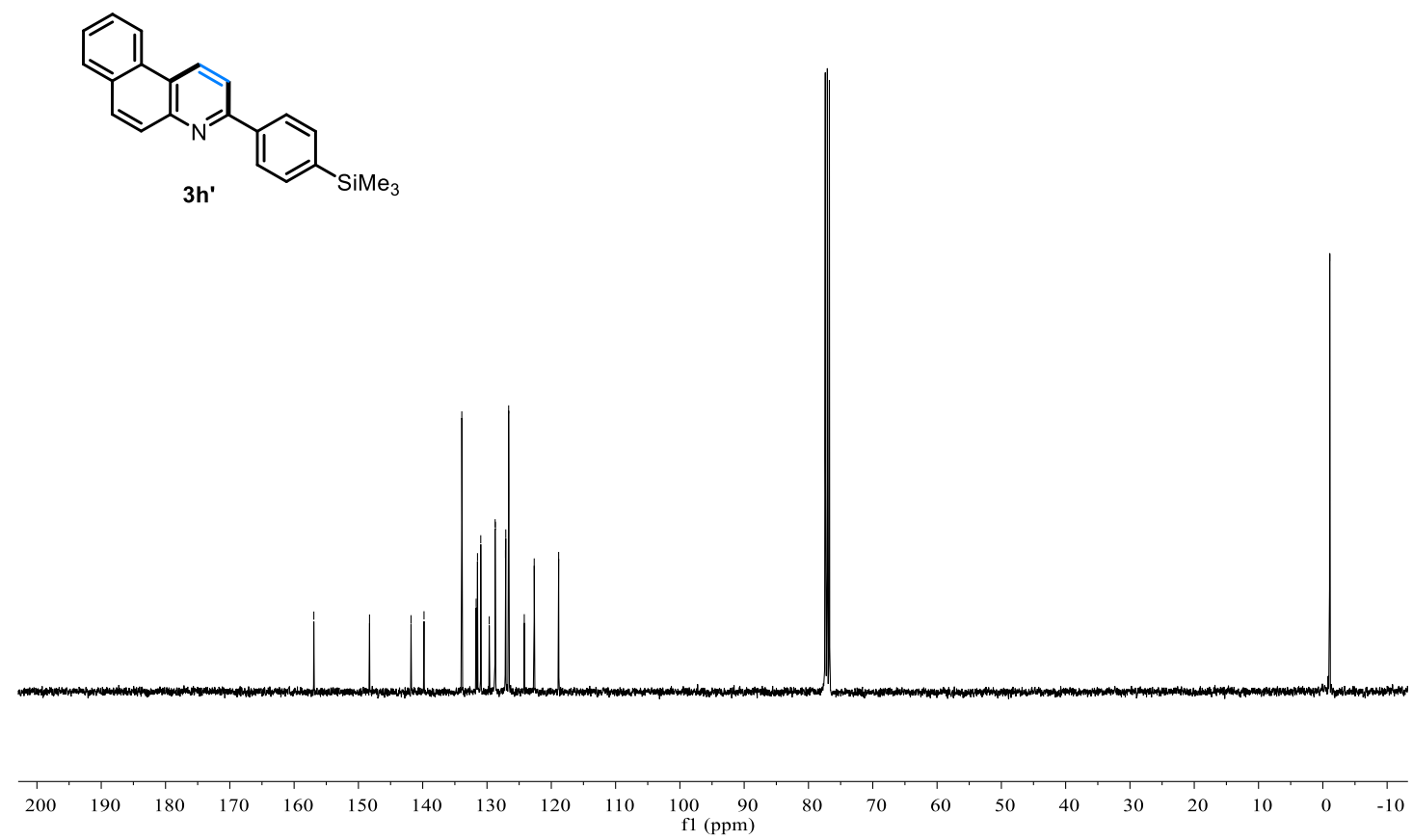

${ }^{1} \mathrm{H}$ NMR of Bi' (400 MHz, DMSO)

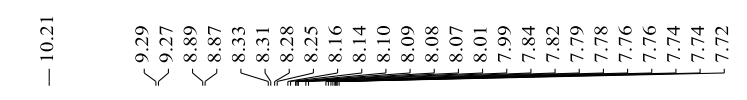

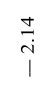
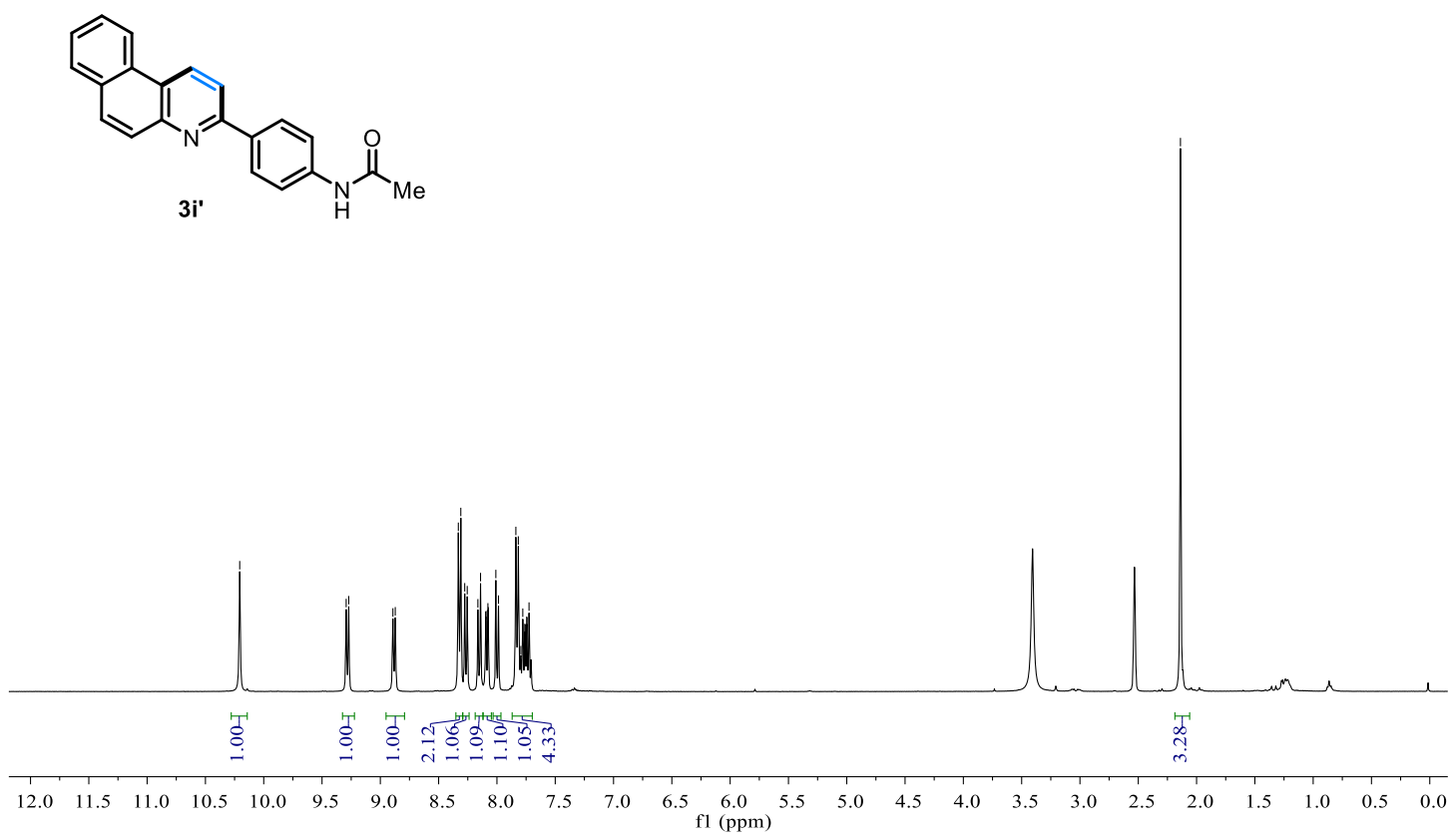

$\mathrm{S} 50$ 
${ }^{13} \mathrm{C}$ NMR of 3i' (100 MHz, DMSO)
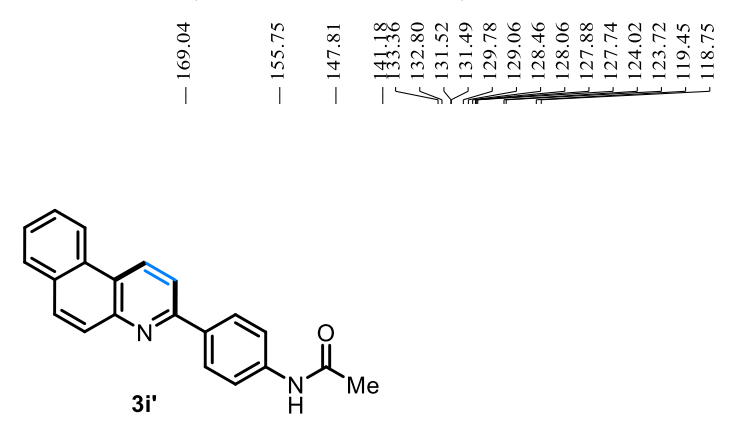
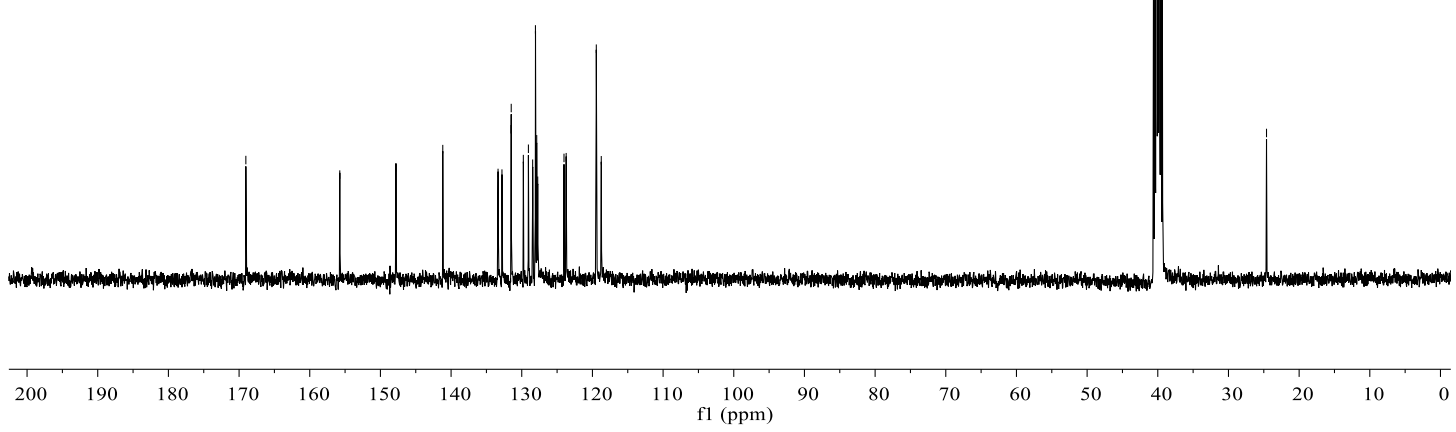

${ }^{1} \mathrm{H}$ NMR of 3j' (400 MHz, DMSO)
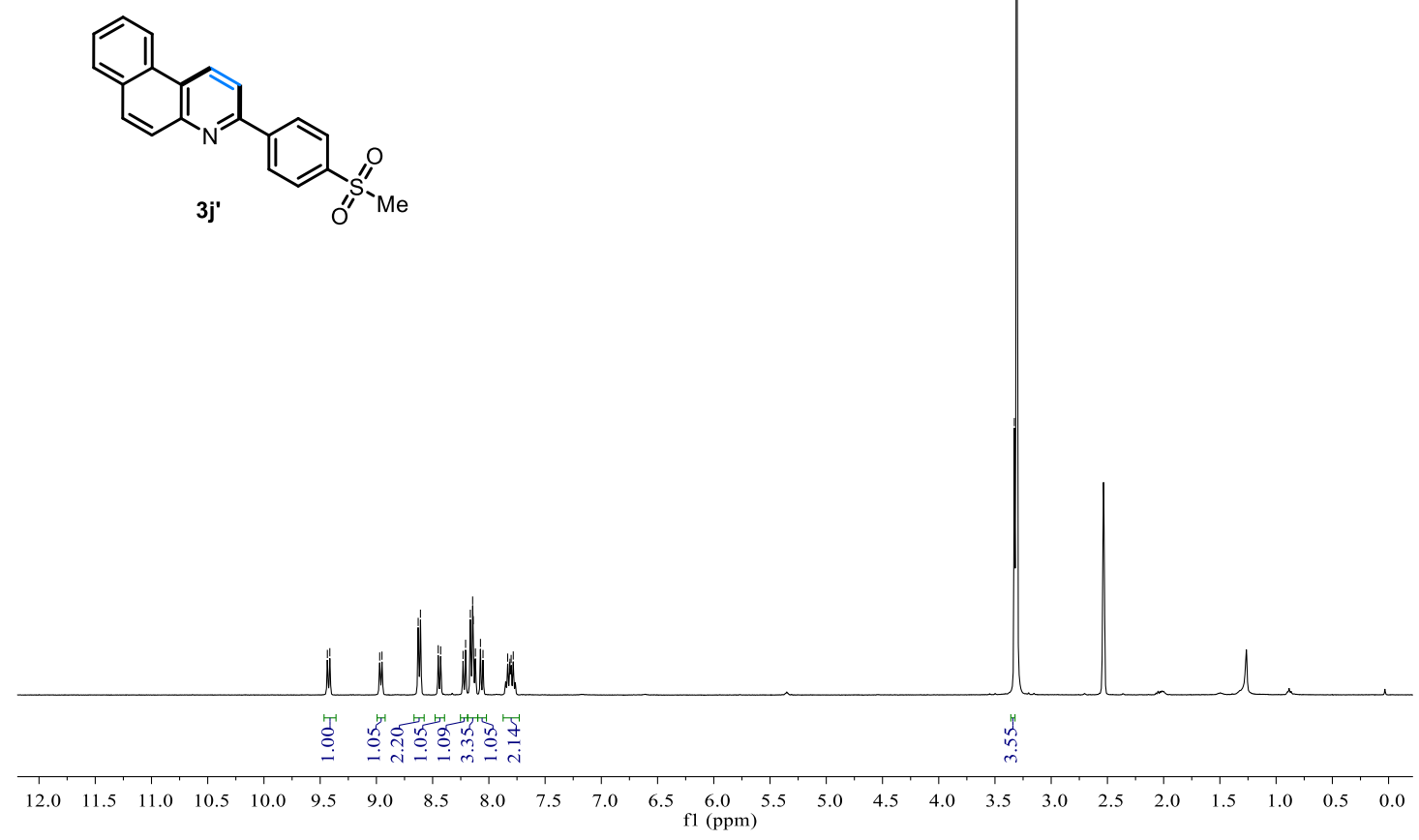

S51 
${ }^{13} \mathrm{C}$ NMR of 3j' (100 MHz, DMSO)

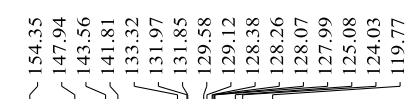

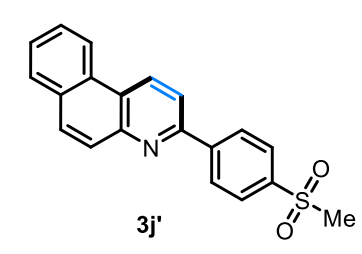

$3 \mathbf{j}^{\prime}$

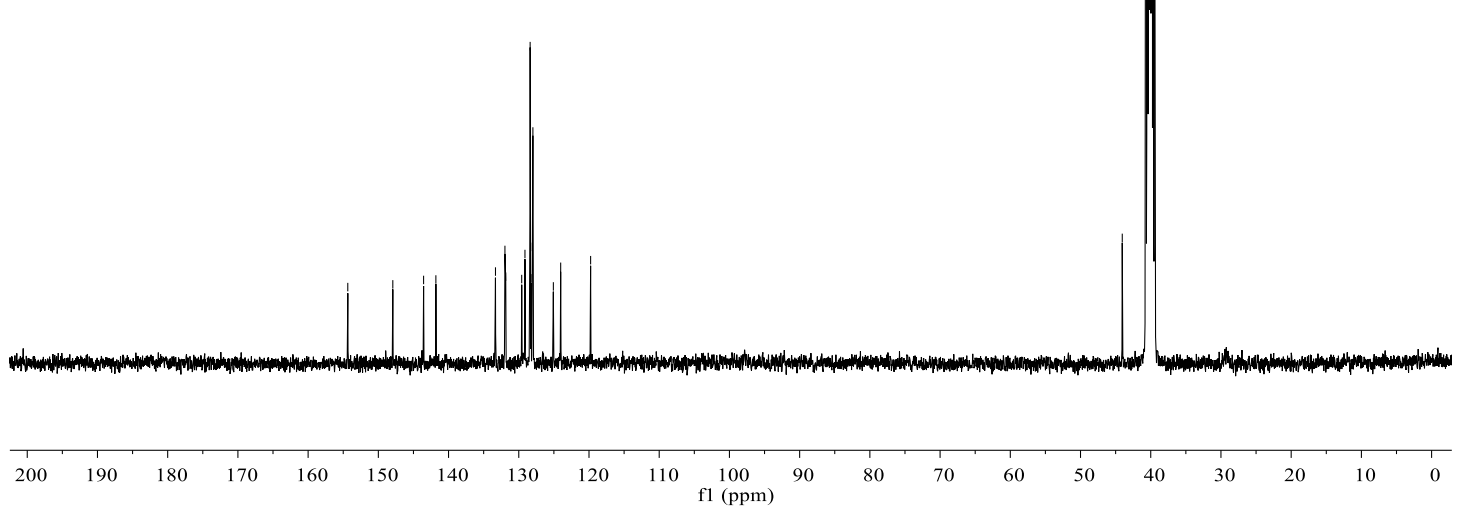

${ }^{1} \mathrm{H} \mathrm{NMR}$ of 3l' (400 MHz, $\mathrm{CDCl}_{3}$ )

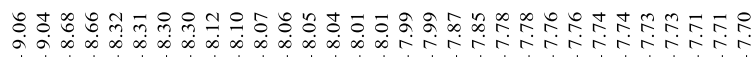
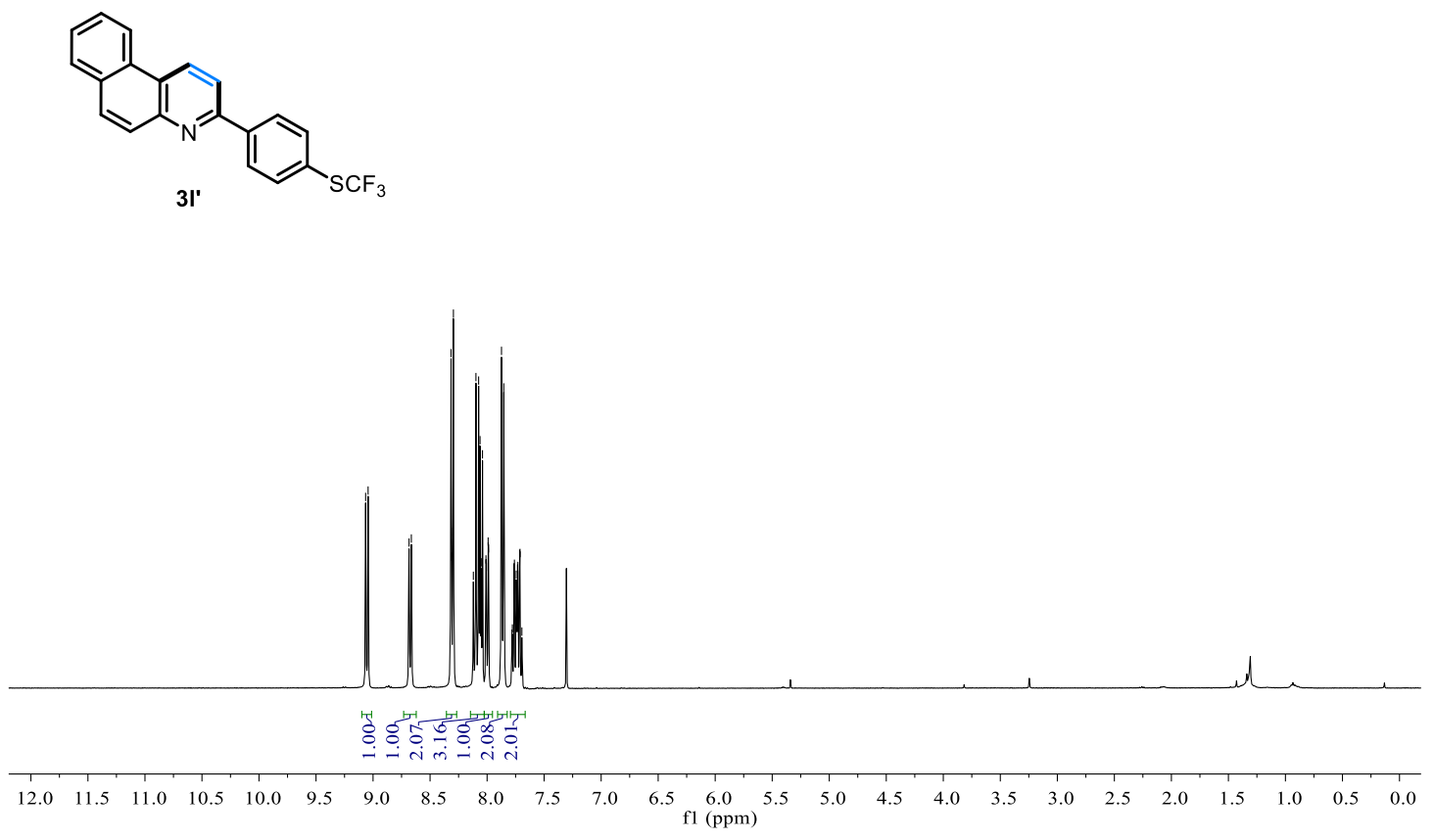

S52 
${ }^{13} \mathrm{C} \mathrm{NMR}$ of $3 \mathbf{l}^{\prime}\left(100 \mathrm{MHz}, \mathrm{CDCl}_{3}\right)$

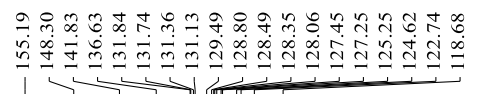
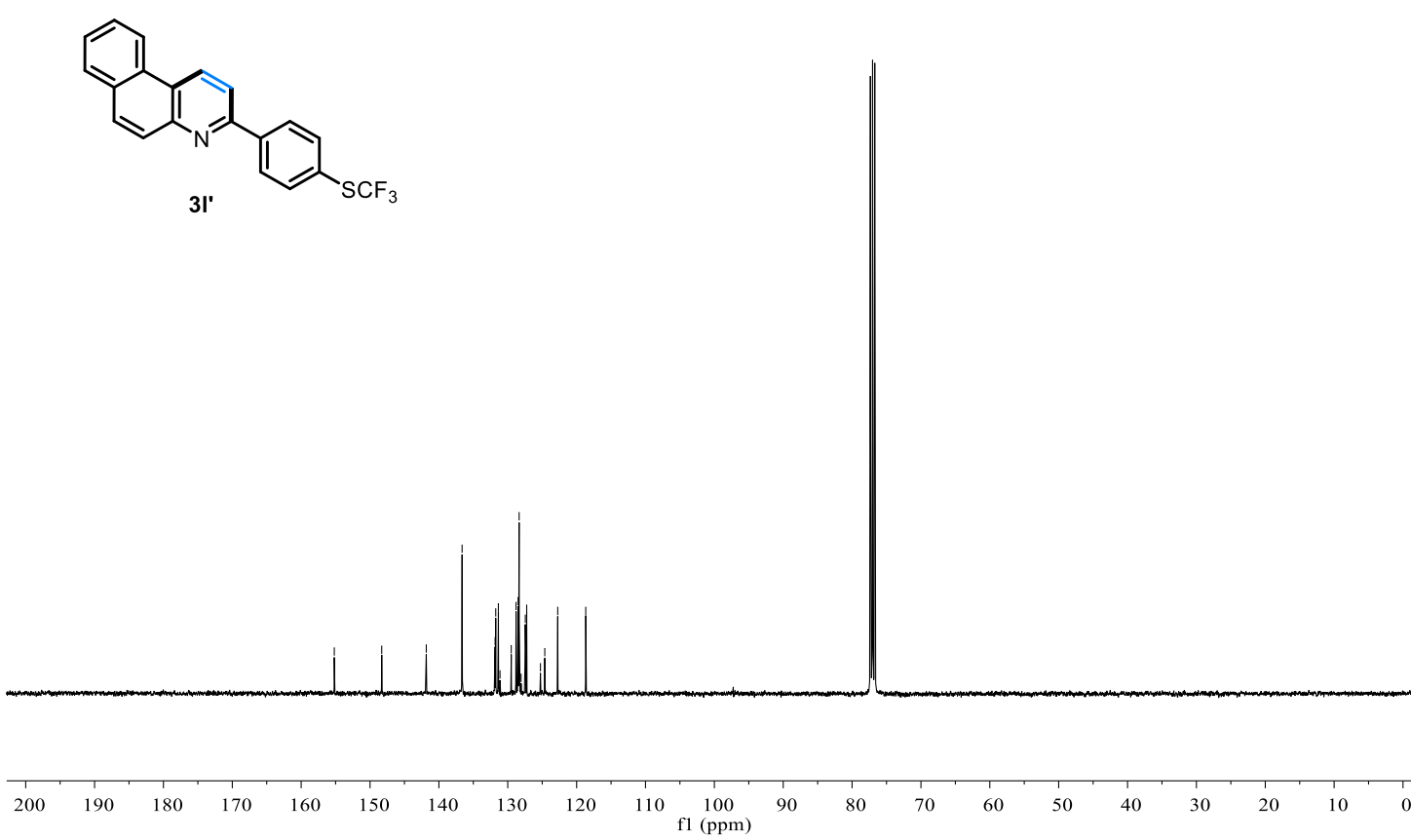

${ }^{19} \mathrm{~F}$ NMR of 3l' (376 MHz, $\left.\mathrm{CDCl}_{3}\right)$
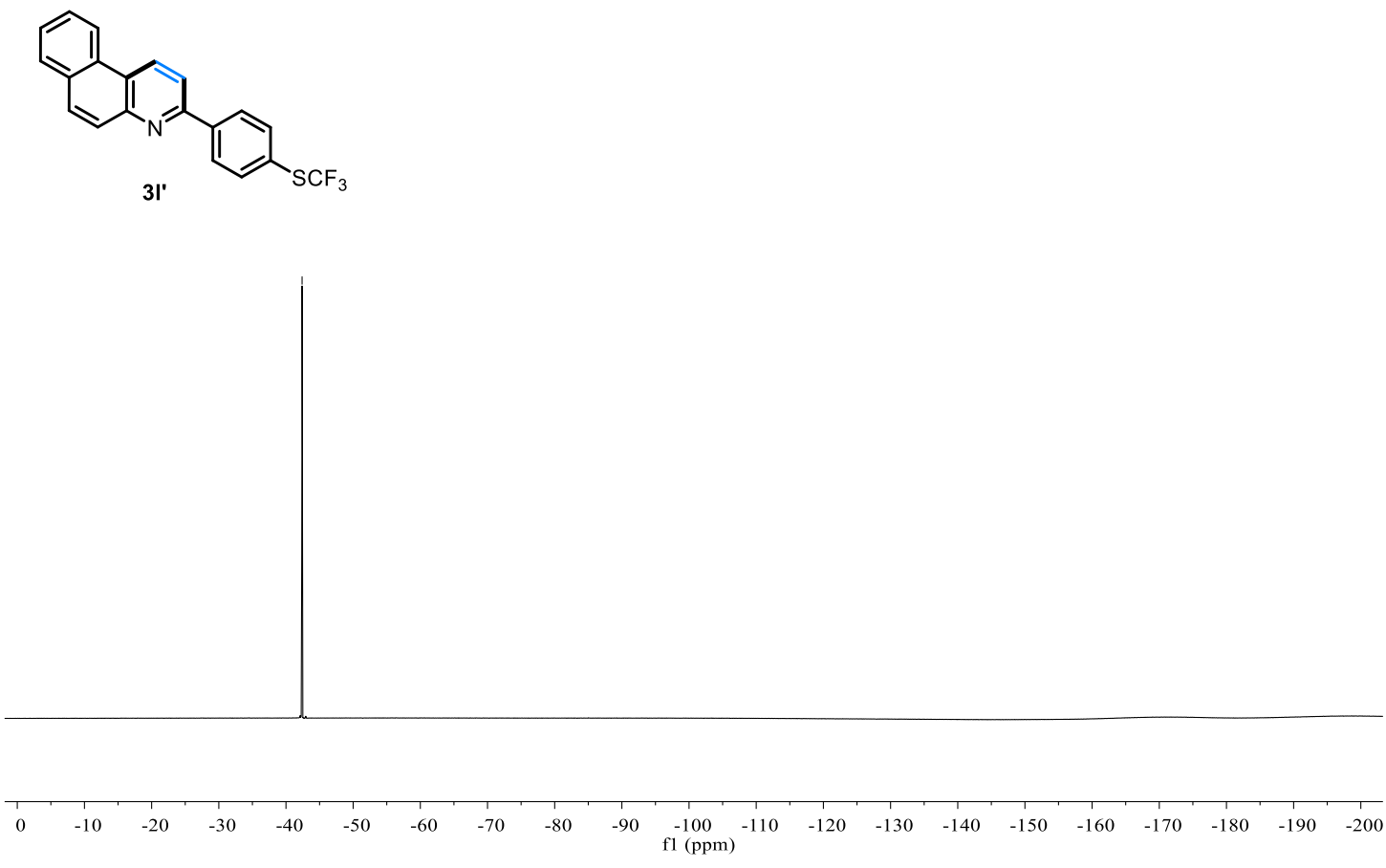

S53 
${ }^{1} \mathrm{H} \mathrm{NMR}$ of 3m' (400 MHz, $\mathrm{CDCl}_{3}$ )

$\underbrace{0}$
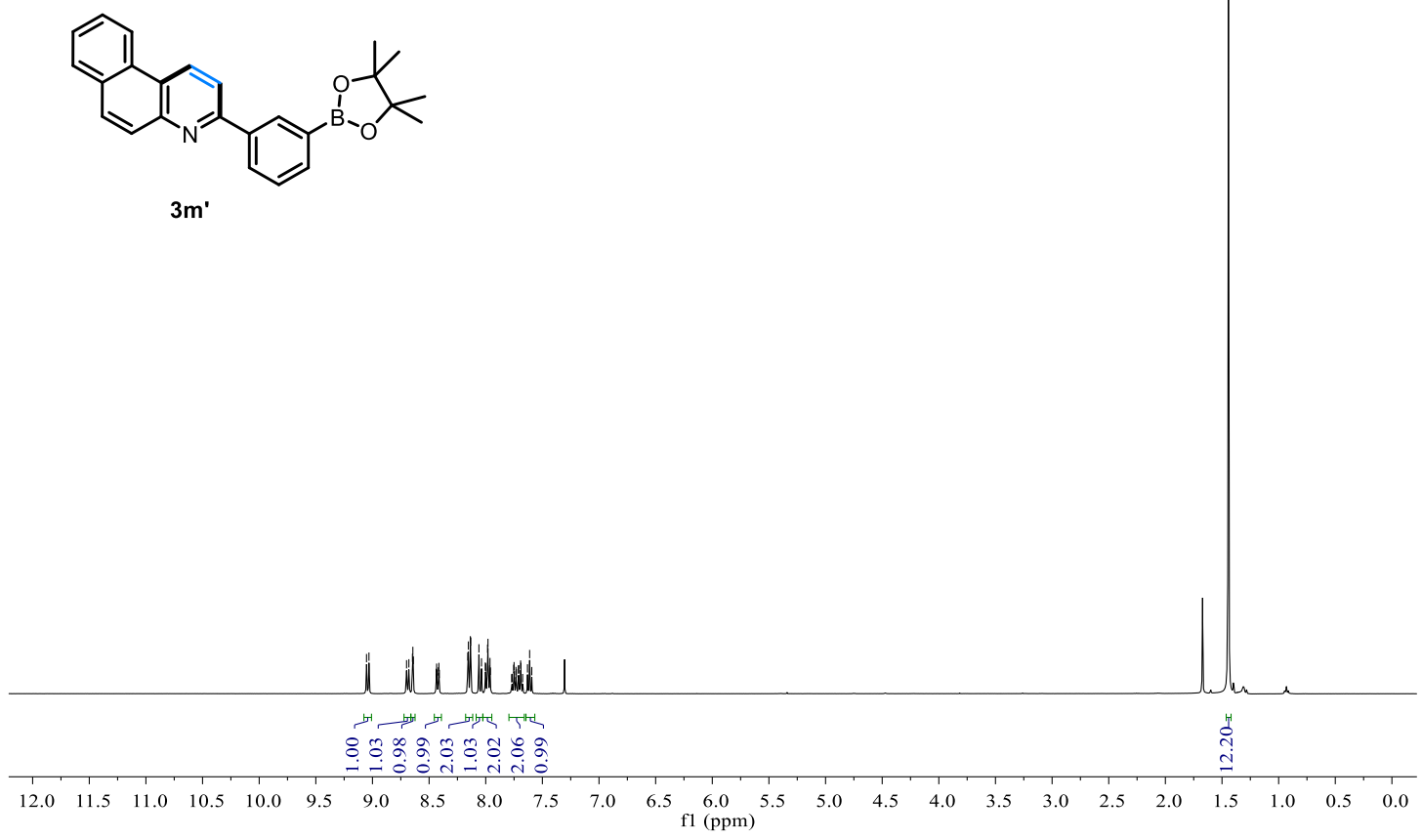

${ }^{13} \mathrm{C}$ NMR of 3m' (100 MHz, $\mathrm{CDCl}_{3}$ )

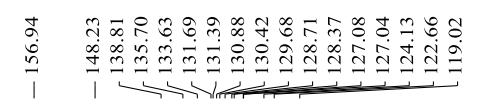

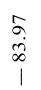

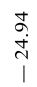
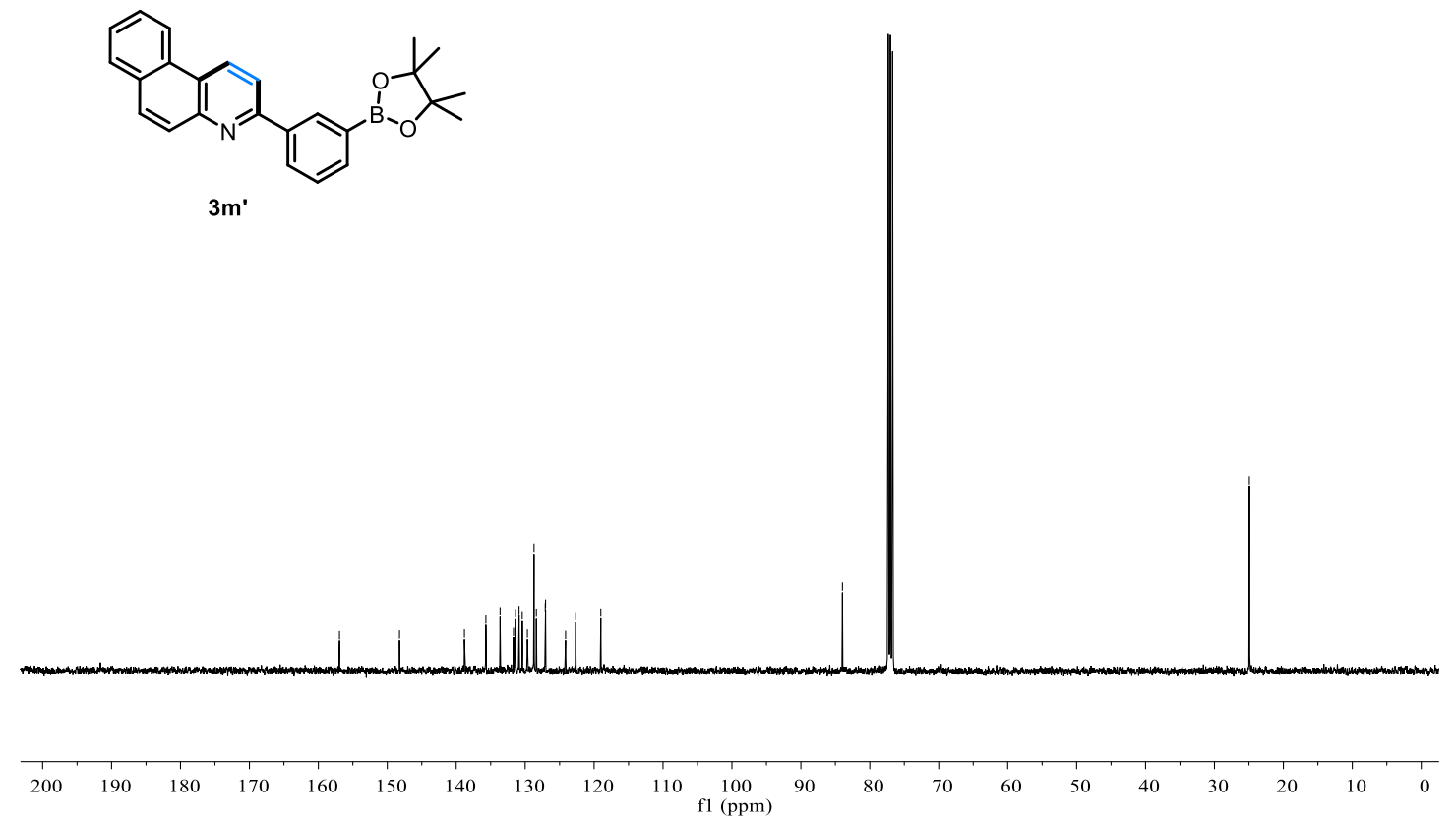

S54 
${ }^{1} \mathrm{H} \mathrm{NMR}$ of 3n' (400 MHz, $\left.\mathrm{CDCl}_{3}\right)$

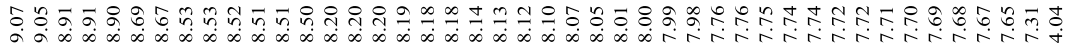

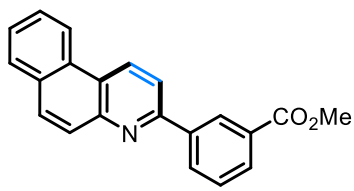

$3 n^{\prime}$

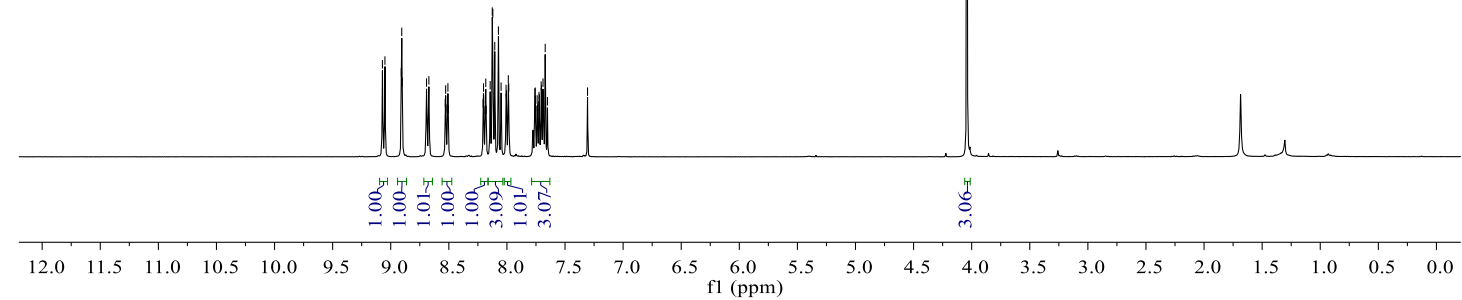

${ }^{13} \mathrm{C}$ NMR of 3n' (100 MHz, $\mathrm{CDCl}_{3}$ )

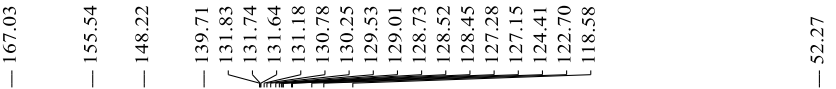

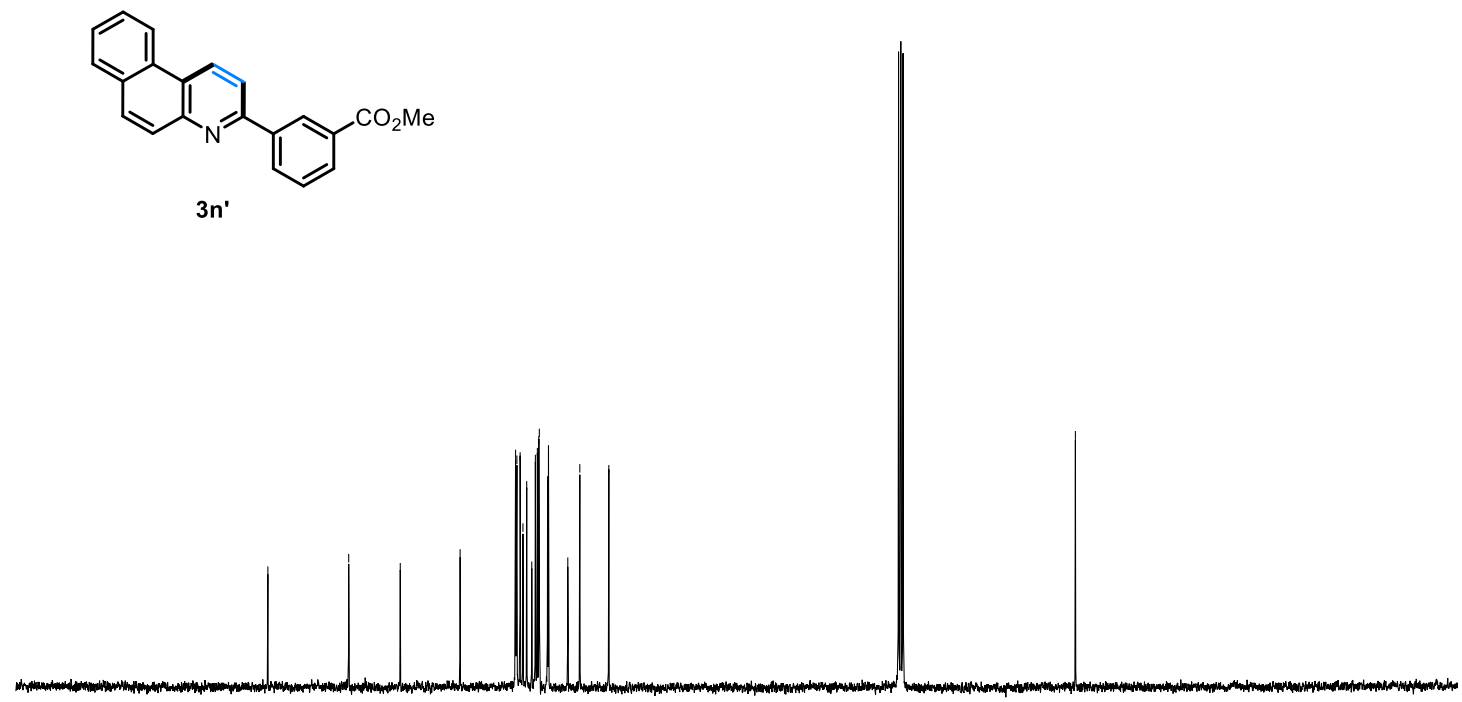

$3 n^{\prime}$

200

190

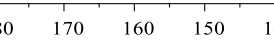

$40 \quad 130$

120

$10 \quad 100$

80

60

30

20 
${ }^{1} \mathrm{H} \mathrm{NMR}$ of 3o' $\left(400 \mathrm{MHz}, \mathrm{CDCl}_{3}\right)$

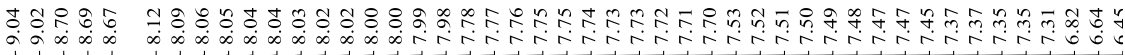

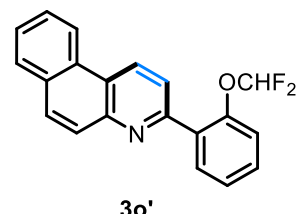

$30^{\prime}$

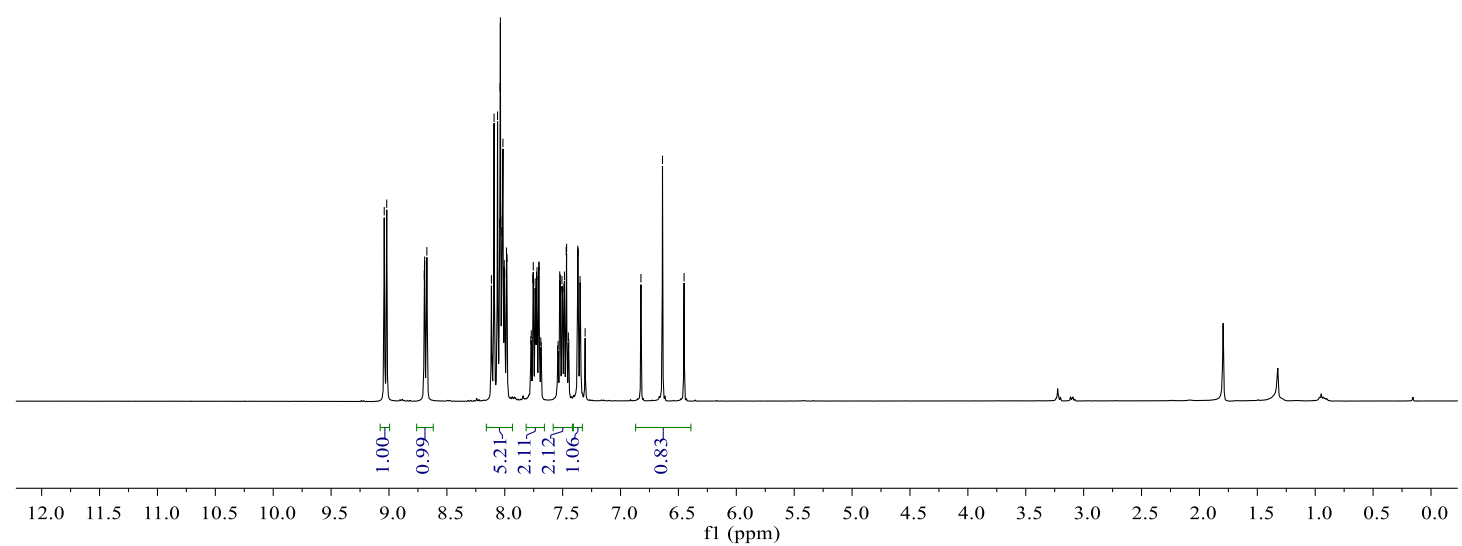

${ }^{13} \mathrm{C}$ NMR of 3o' (100 MHz, $\left.\mathrm{CDCl}_{3}\right)$

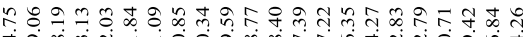

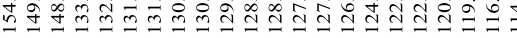

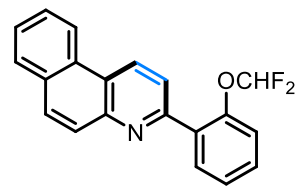

3o'

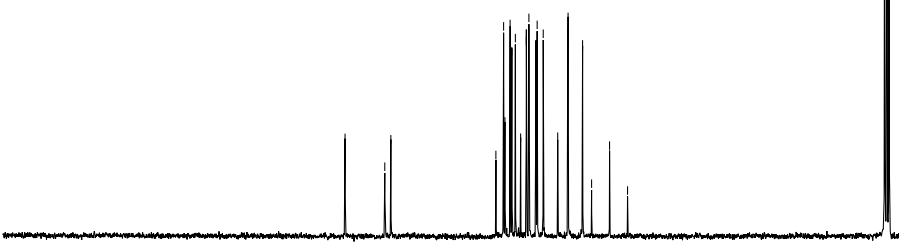

$\begin{array}{lllllllllllllllllllll}200 & 190 & 180 & 170 & 160 & 150 & 140 & 130 & 120 & 110 & 100 & 90 & 80 & 70 & 60 & 50 & 40 & 30 & 20 & 10 & 0\end{array}$ 
${ }^{19} \mathrm{~F}$ NMR of 3o' (376 MHz, $\left.\mathrm{CDCl}_{3}\right)$

吕
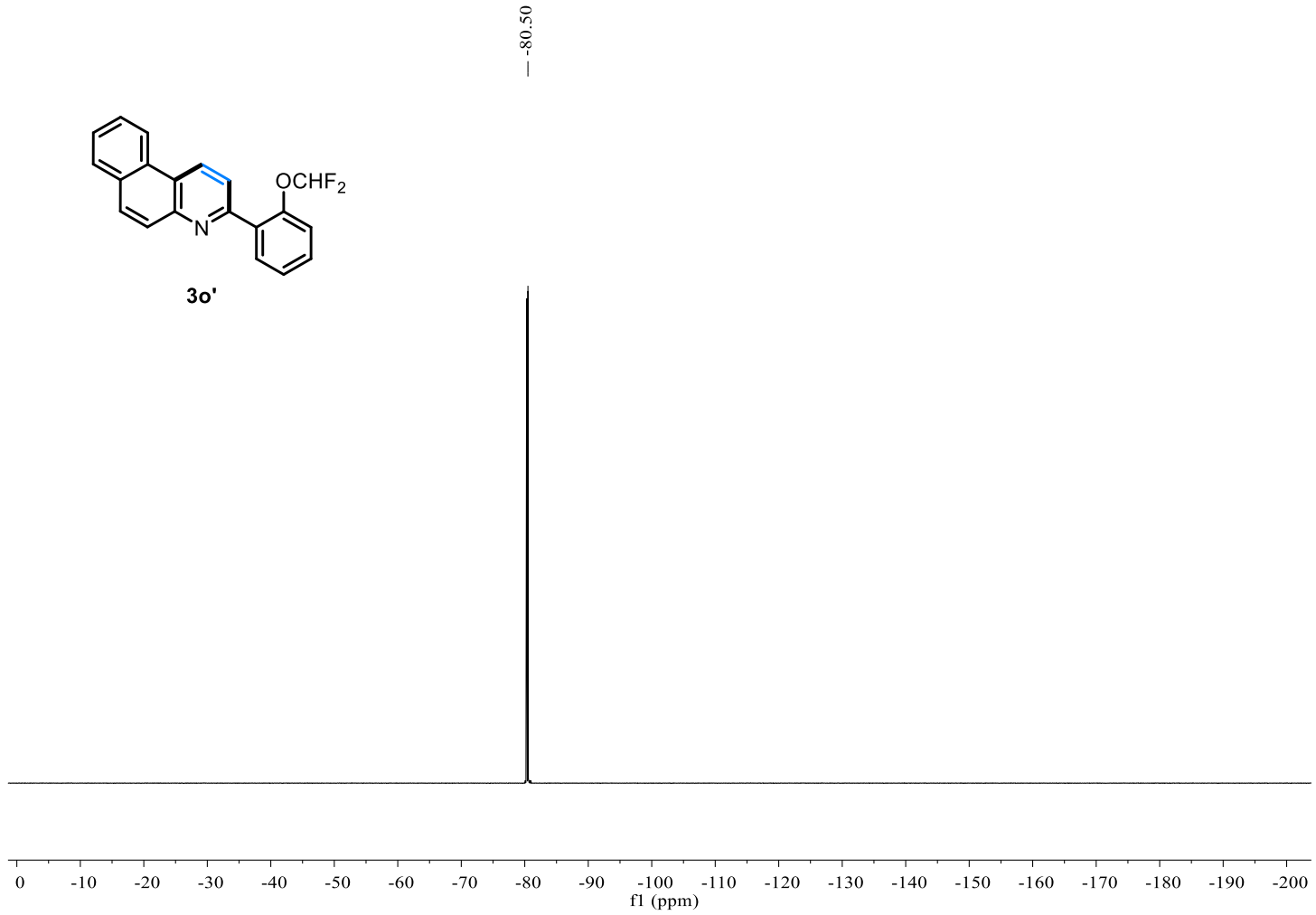

${ }^{1} \mathrm{H}$ NMR of 3p' (400 MHz, $\left.\mathrm{CDCl}_{3}\right)$
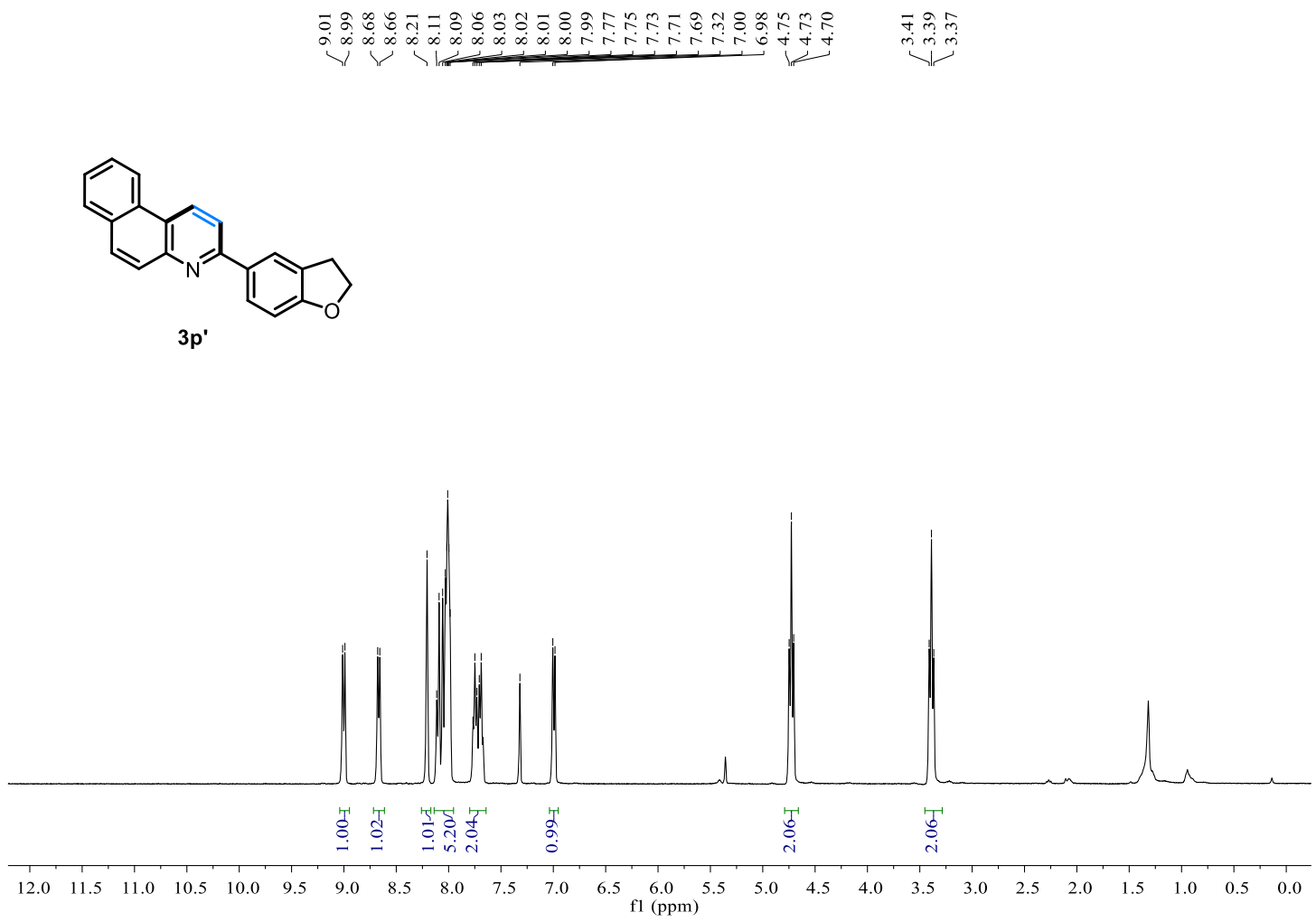

S57 
${ }^{13} \mathrm{C}$ NMR of 3p' $\left(100 \mathrm{MHz}, \mathrm{CDCl}_{3}\right)$

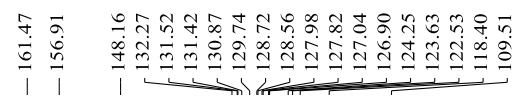

$\stackrel{\infty}{i}$

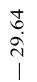
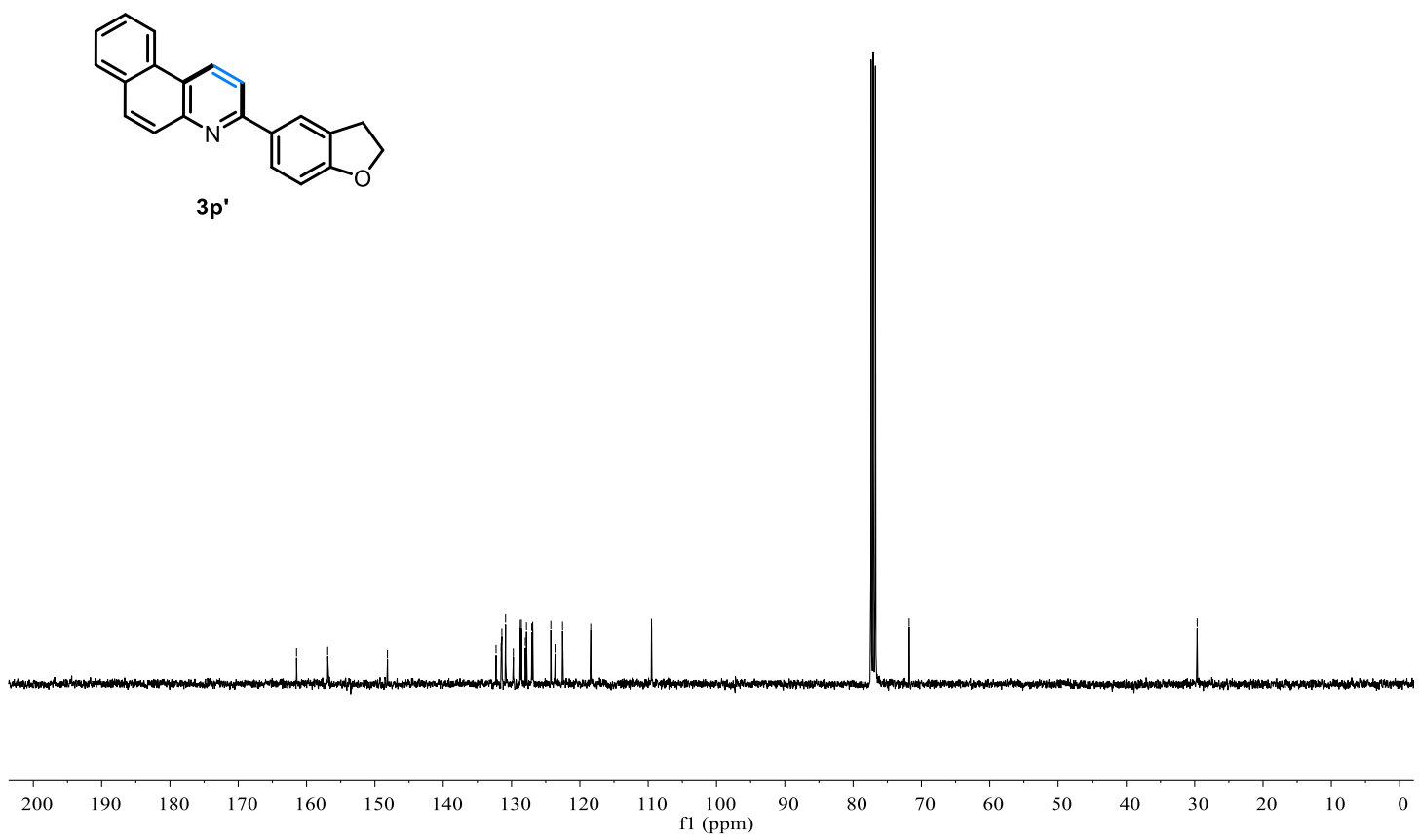

${ }^{1} \mathrm{H} \mathrm{NMR}$ of $\mathbf{3 q}$ ' (400 MHz, $\mathrm{CDCl}_{3}$ )

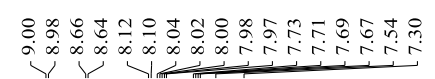

$\stackrel{\infty}{+\infty}$
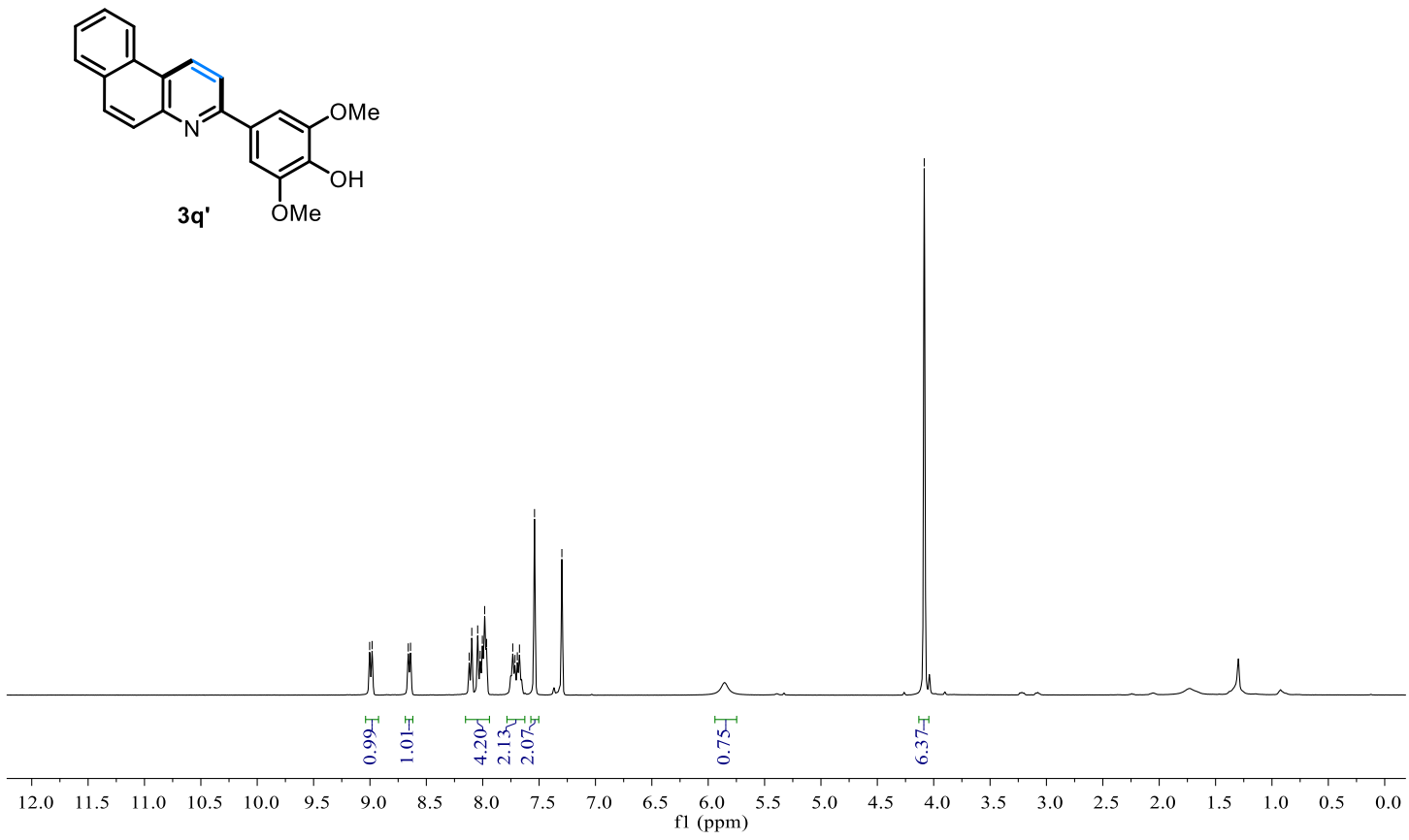

S58 
${ }^{13} \mathrm{C}$ NMR of $\mathbf{3 q}$ ' (100 MHz, $\left.\mathrm{CDCl}_{3}\right)$

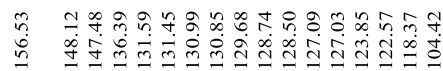

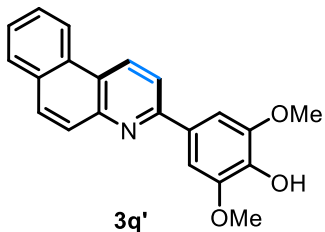

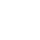


${ }^{13} \mathrm{C}$ NMR of 3r' (100 MHz, $\left.\mathrm{CDCl}_{3}\right)$
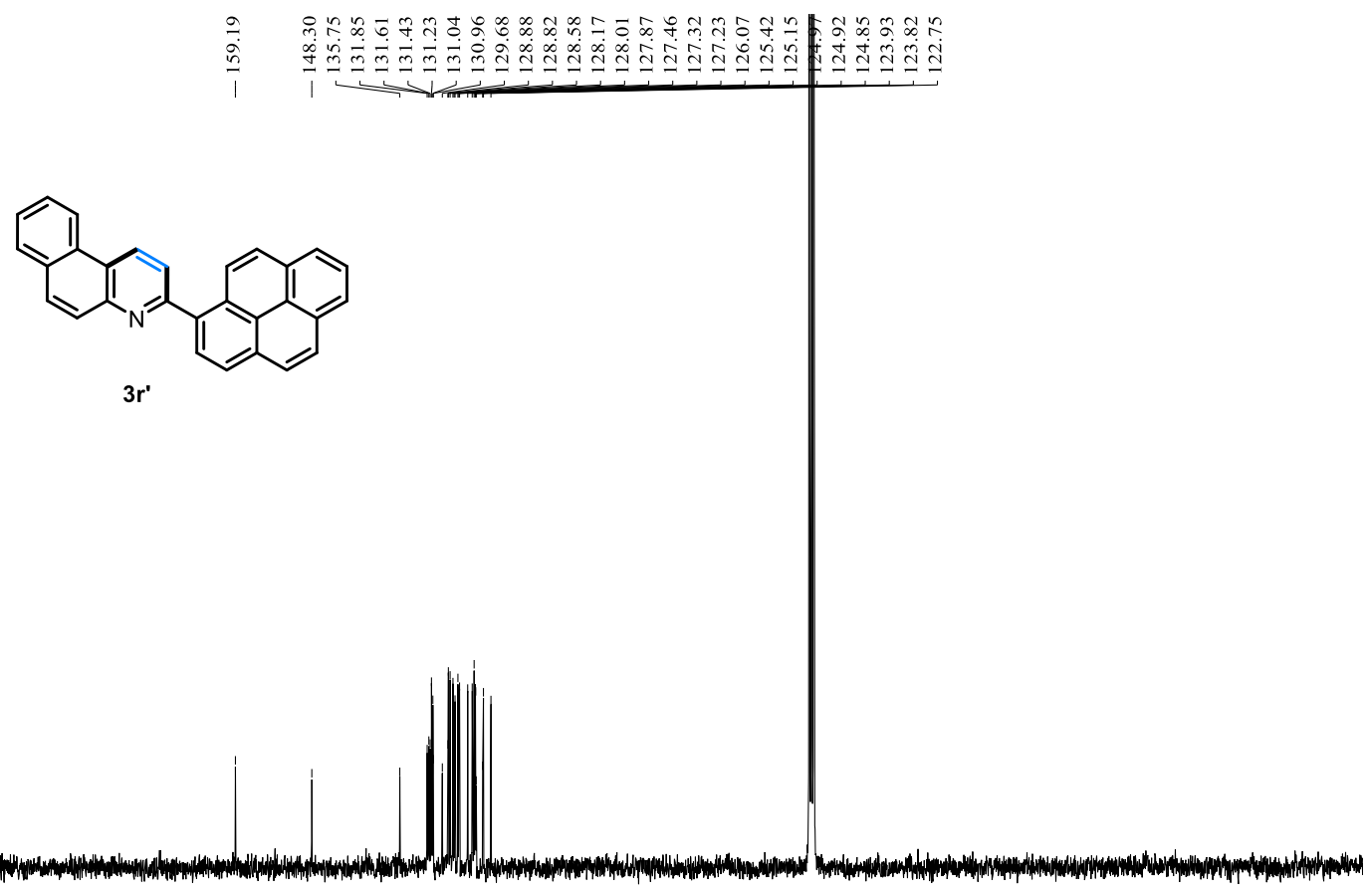

$3 r^{\prime \prime}$

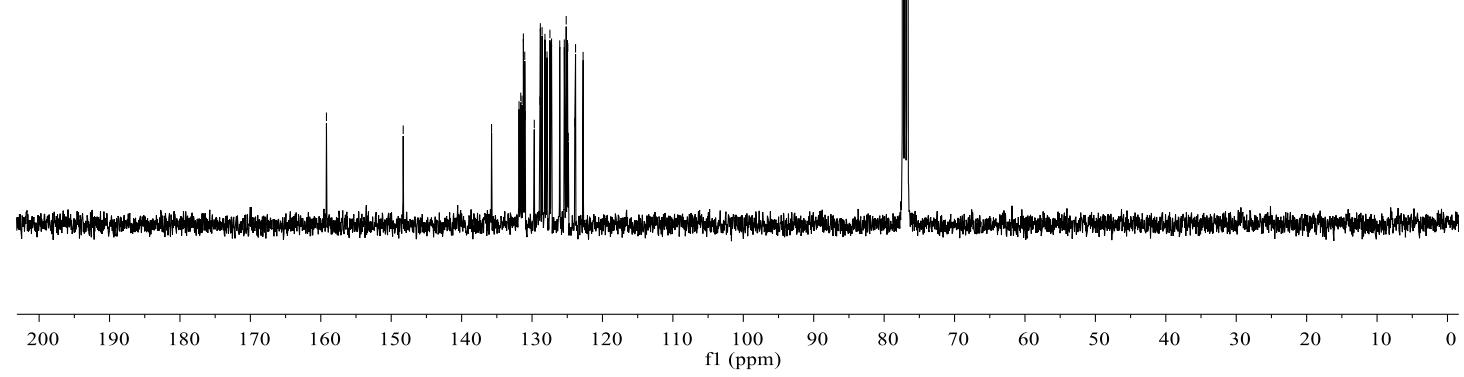

${ }^{1} \mathrm{H}$ NMR of 3s' (400 MHz, $\mathrm{CDCl}_{3}$ )

5
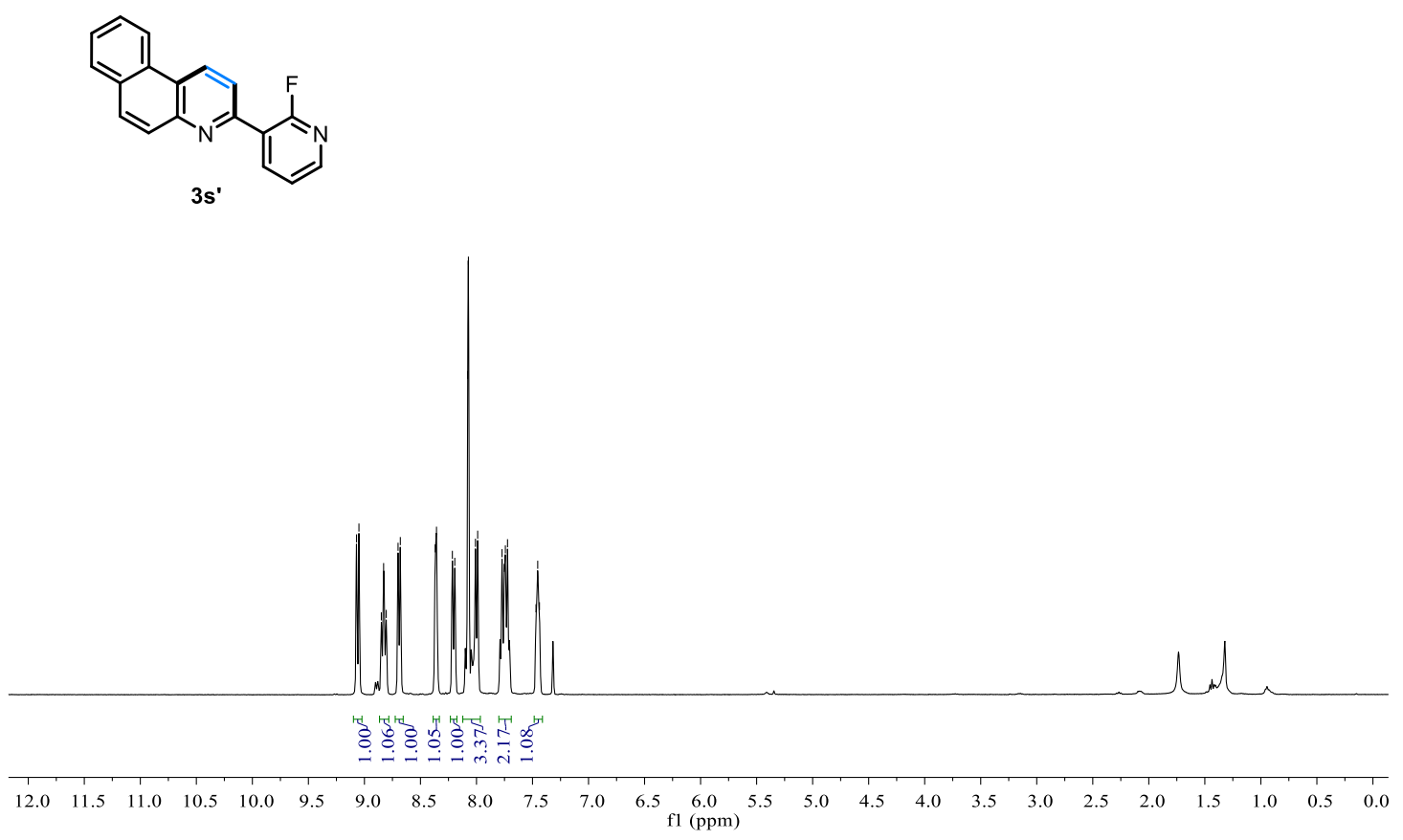

S60 
${ }^{13} \mathrm{C} \mathrm{NMR}$ of $3 \mathbf{s}^{\prime}\left(100 \mathrm{MHz}, \mathrm{CDCl}_{3}\right)$

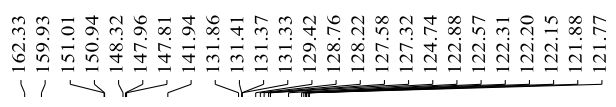
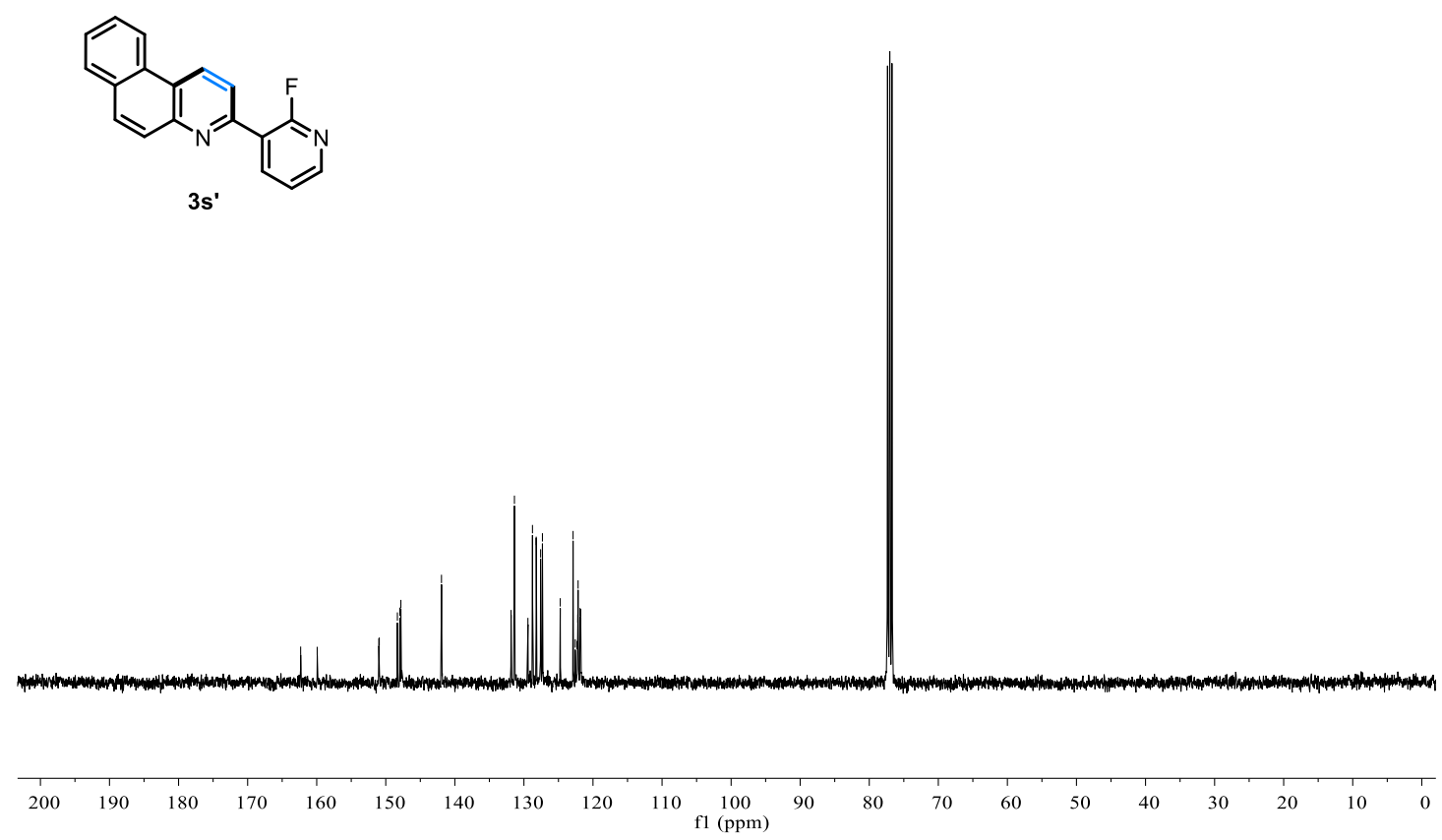

${ }^{19} \mathrm{~F}$ NMR of 3s' (376 MHz, $\mathrm{CDCl}_{3}$ )

亩

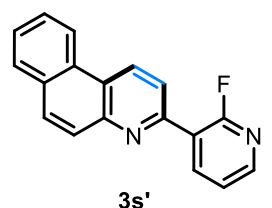

$3 s^{\prime}$

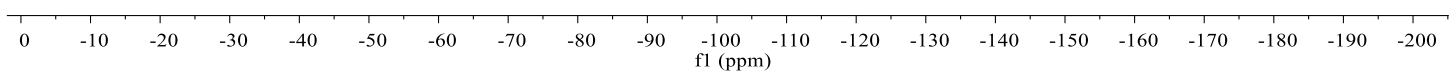

S61 
${ }^{1} \mathrm{H}$ NMR of 3t' $\left(400 \mathrm{MHz}, \mathrm{CDCl}_{3}\right)$

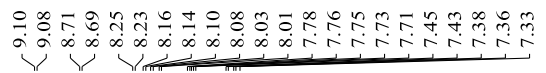
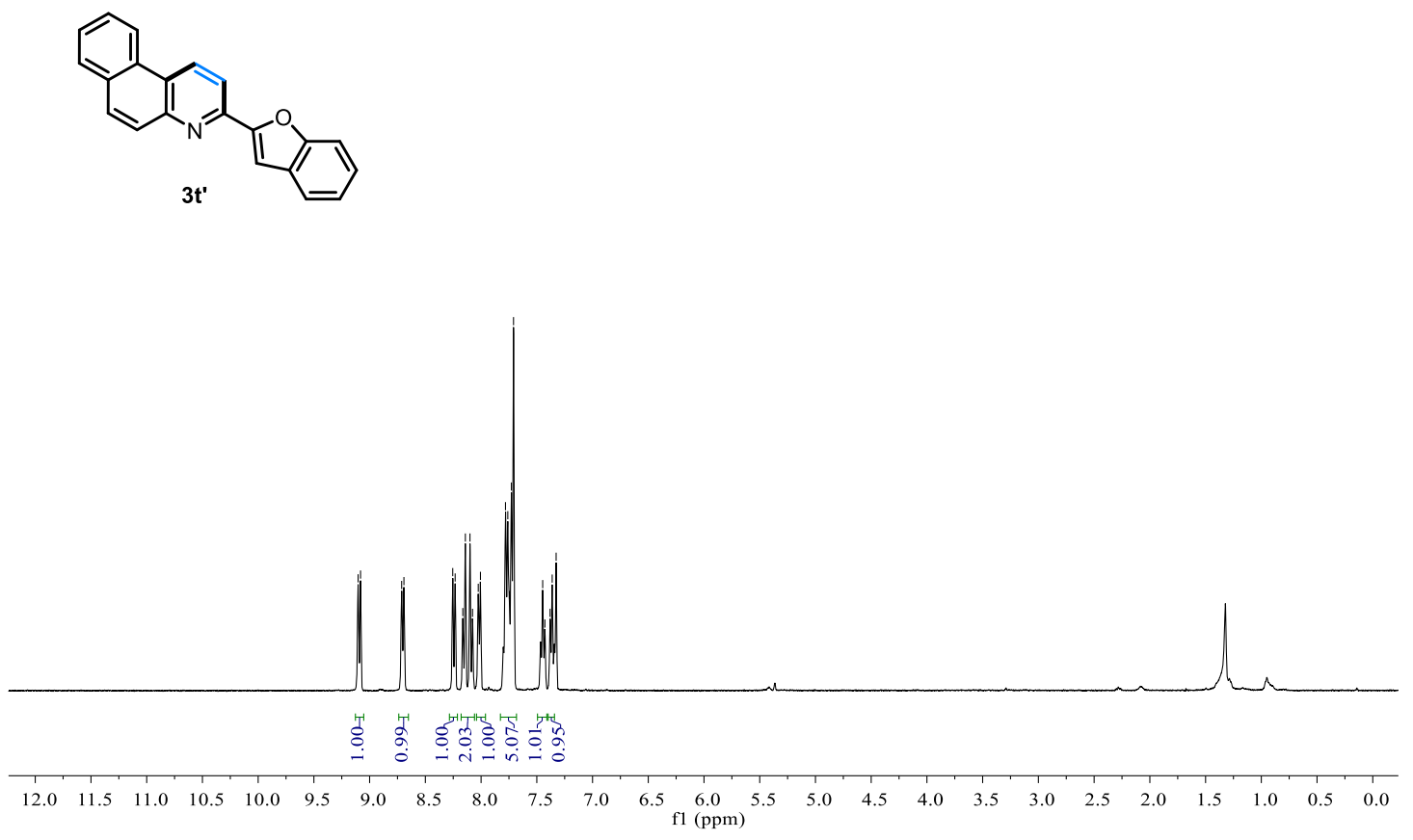

${ }^{13} \mathrm{C}$ NMR of $\mathbf{3 t}$ ' (100 MHz, $\left.\mathrm{CDCl}_{3}\right)$

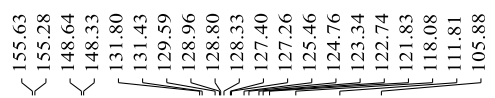
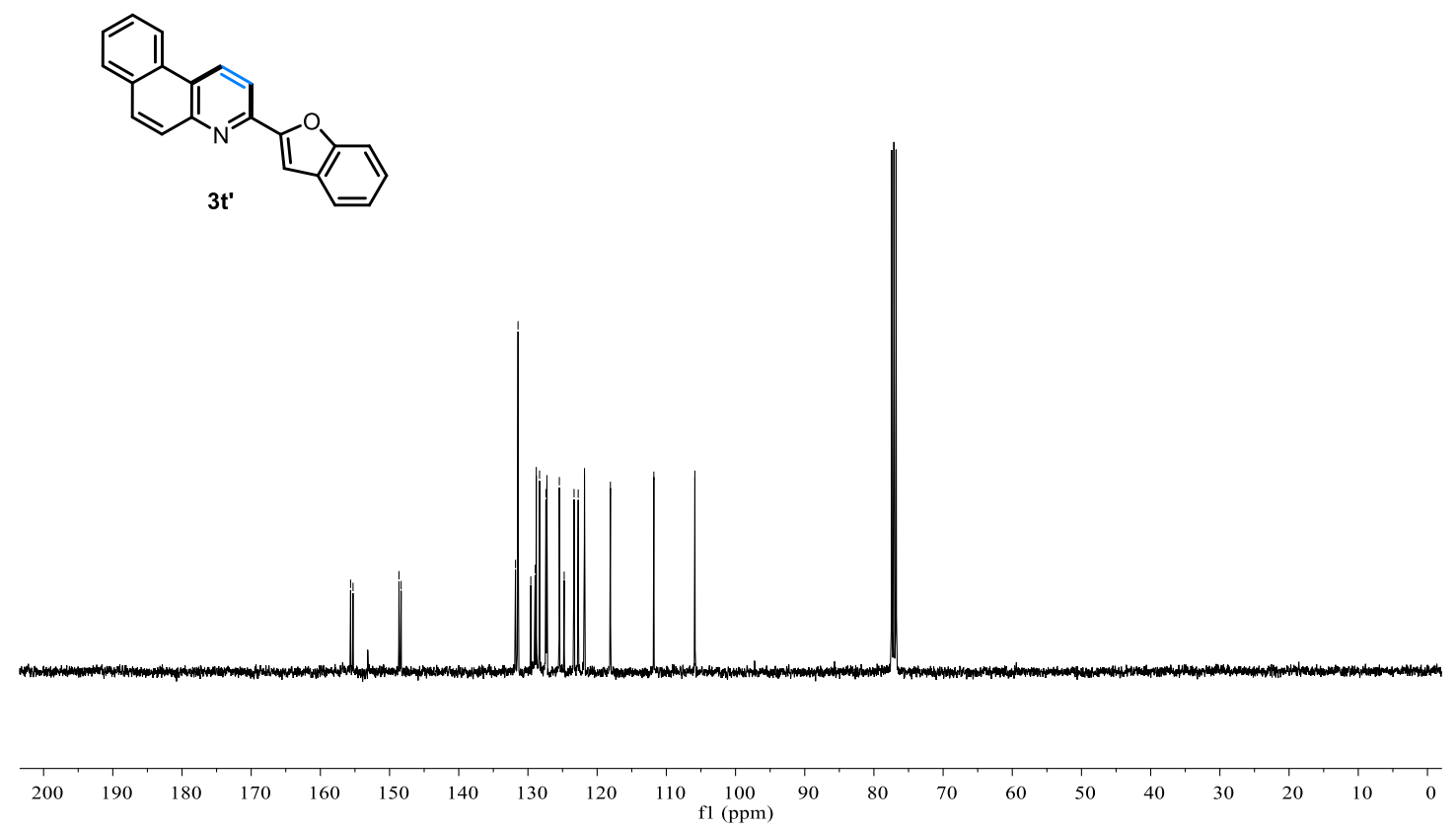

S62 
${ }^{1} \mathrm{H}$ NMR of 6 (400 MHz, DMSO)

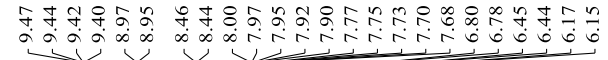
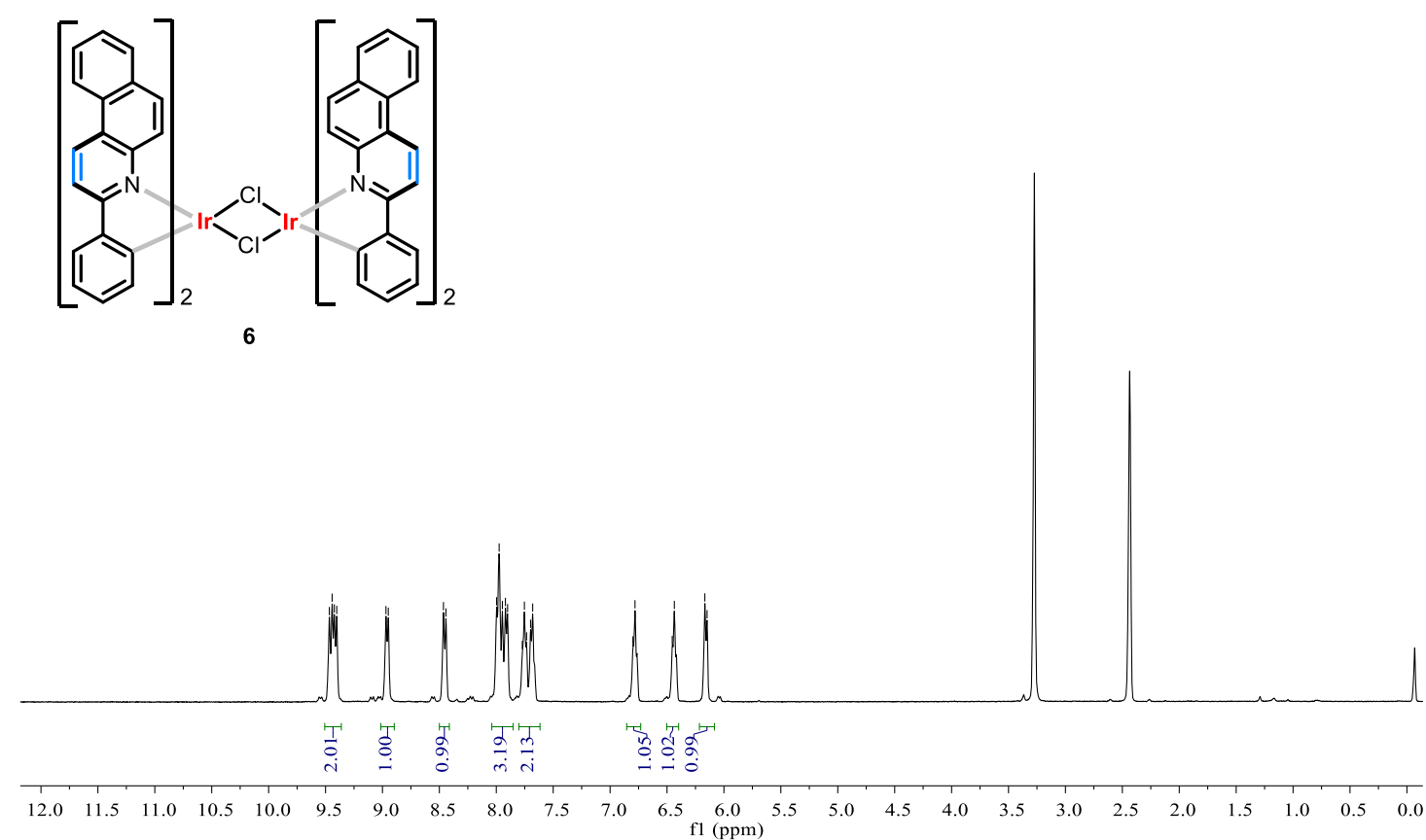

${ }^{13} \mathrm{C}$ NMR of 6 (100 MHz, DMSO)

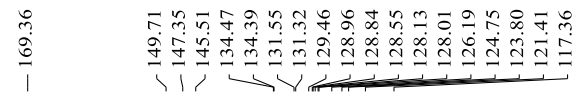
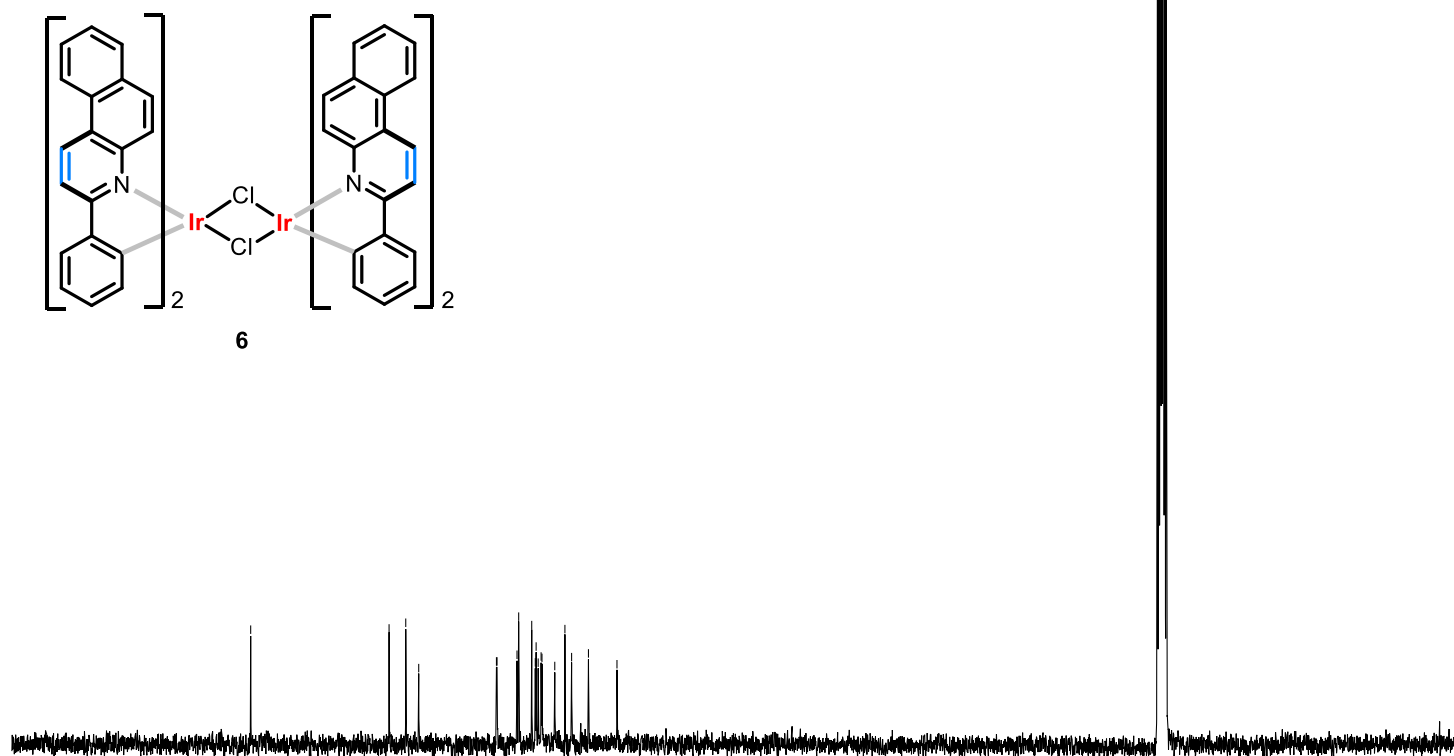

$\begin{array}{lllllllllllllllllllll}200 & 190 & 180 & 170 & 160 & 150 & 140 & 130 & 120 & 110 & \begin{array}{l}100 \\ 100\end{array} & 90 & 80 & 70 & 60 & 50 & 40 & 30 & 20 & 10 & 0\end{array}$ 
${ }^{1} \mathrm{H}$ NMR of $7\left(400 \mathrm{MHz}, \mathrm{CDCl}_{3}\right)$

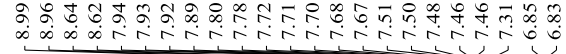
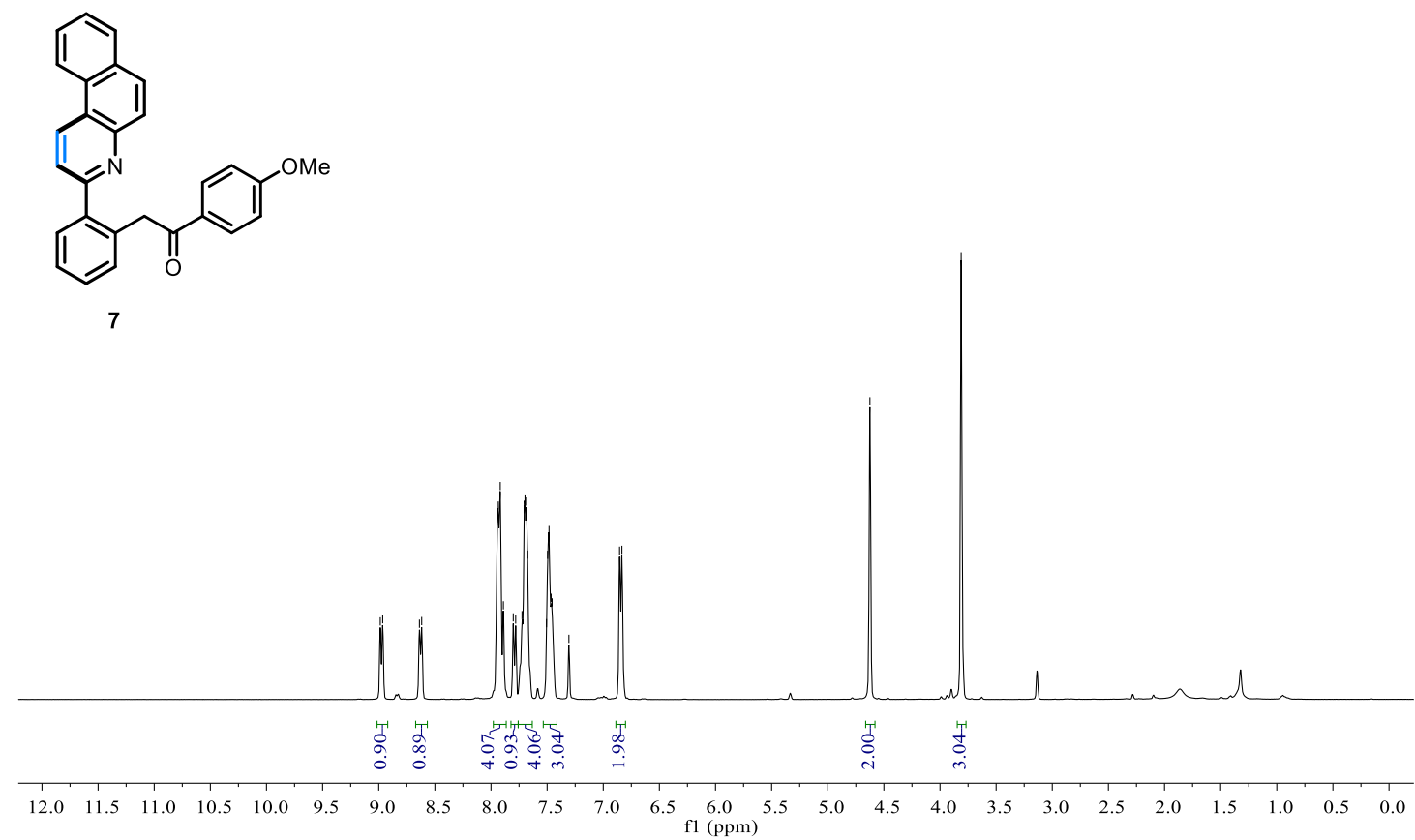

${ }^{13} \mathrm{C}$ NMR of $7\left(100 \mathrm{MHz}, \mathrm{CDCl}_{3}\right)$
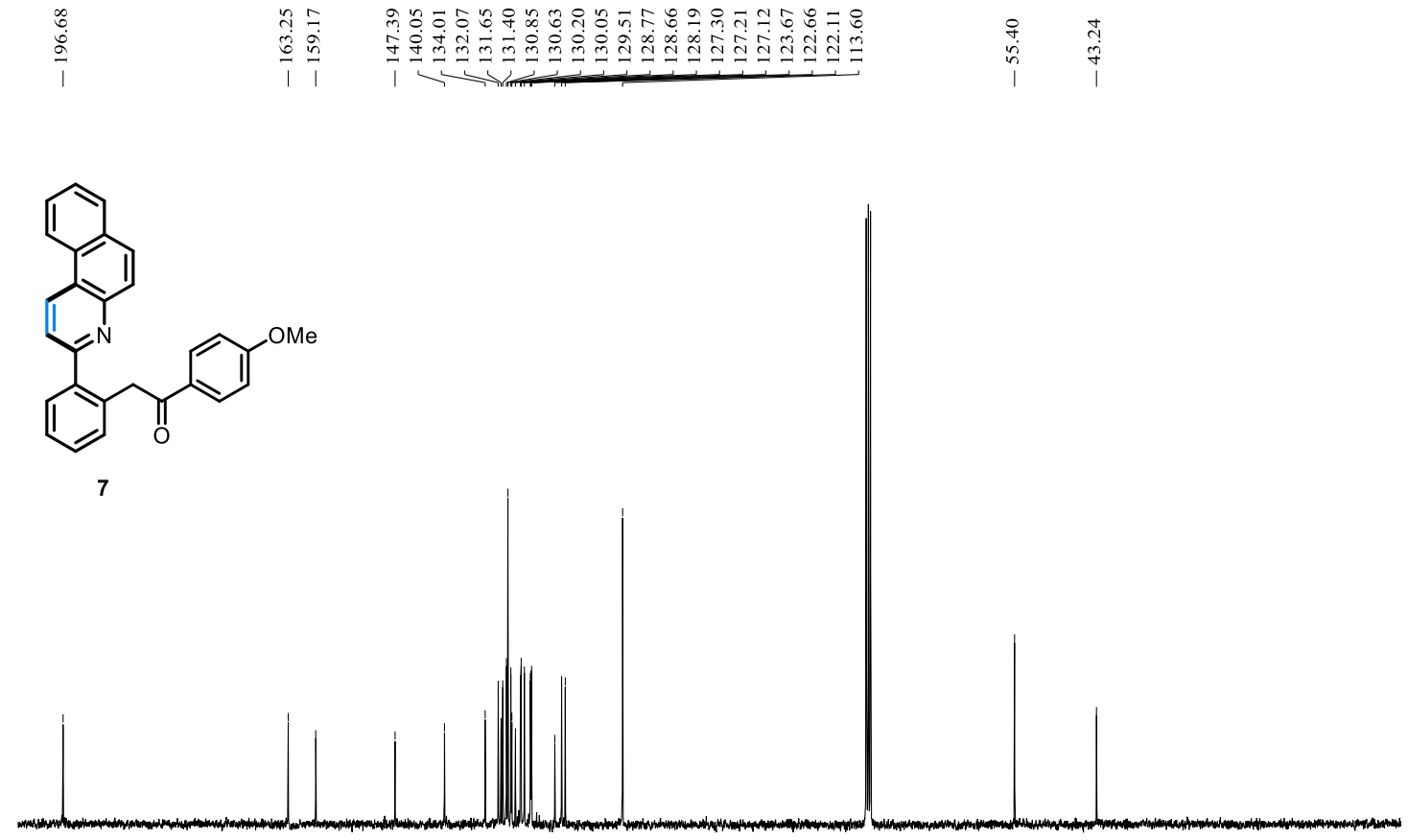

$\begin{array}{llllllllllllllllllllll}200 & 190 & 180 & 170 & 160 & 150 & 140 & 130 & 120 & 110 & 100 & 90 & 80 & 70 & 60 & 50 & 40 & 30 & 20 & 10 & 0\end{array}$ 

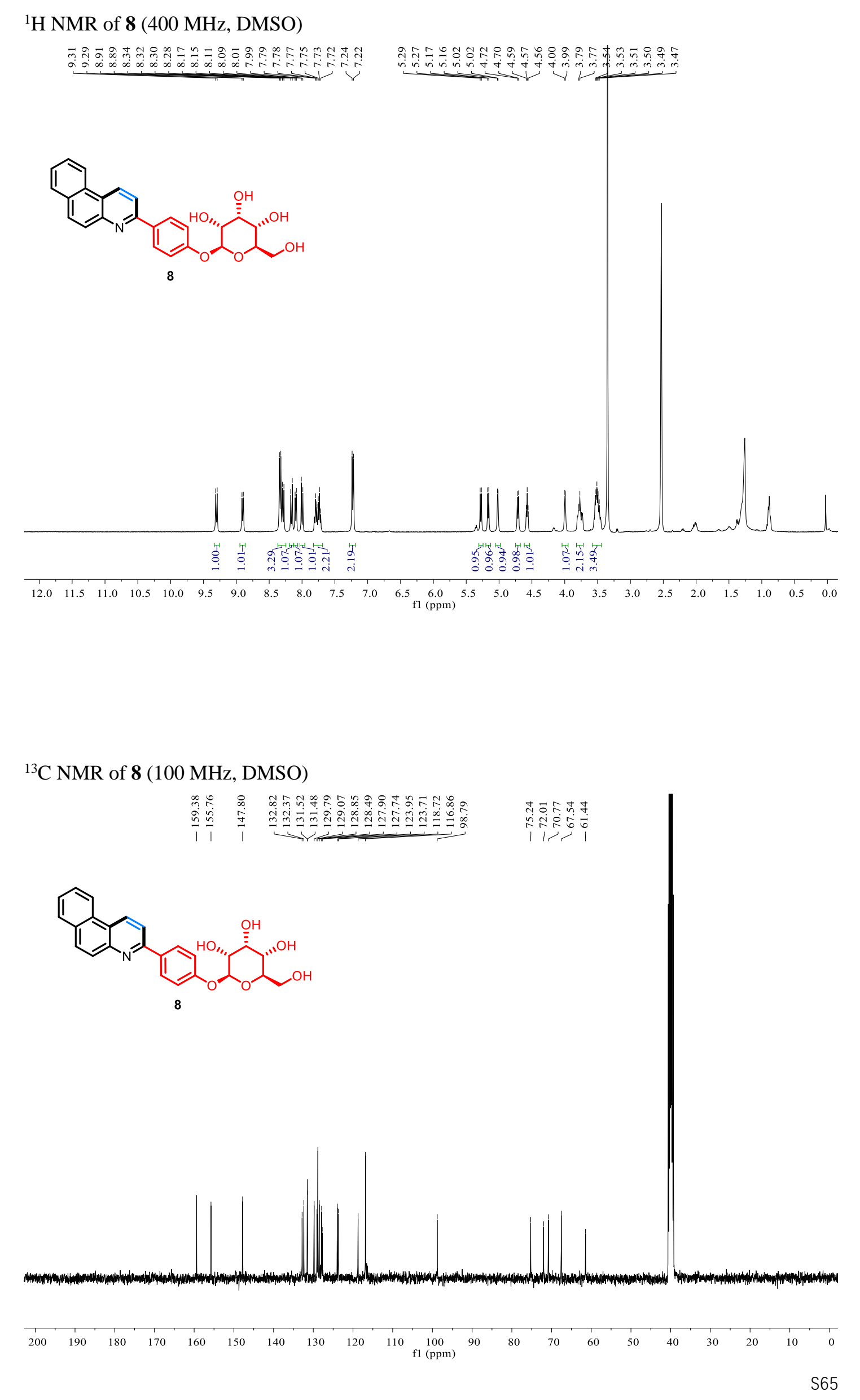
${ }^{1} \mathrm{H}$ NMR of $9\left(400 \mathrm{MHz}, \mathrm{CDCl}_{3}\right)$

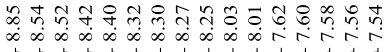

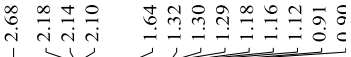
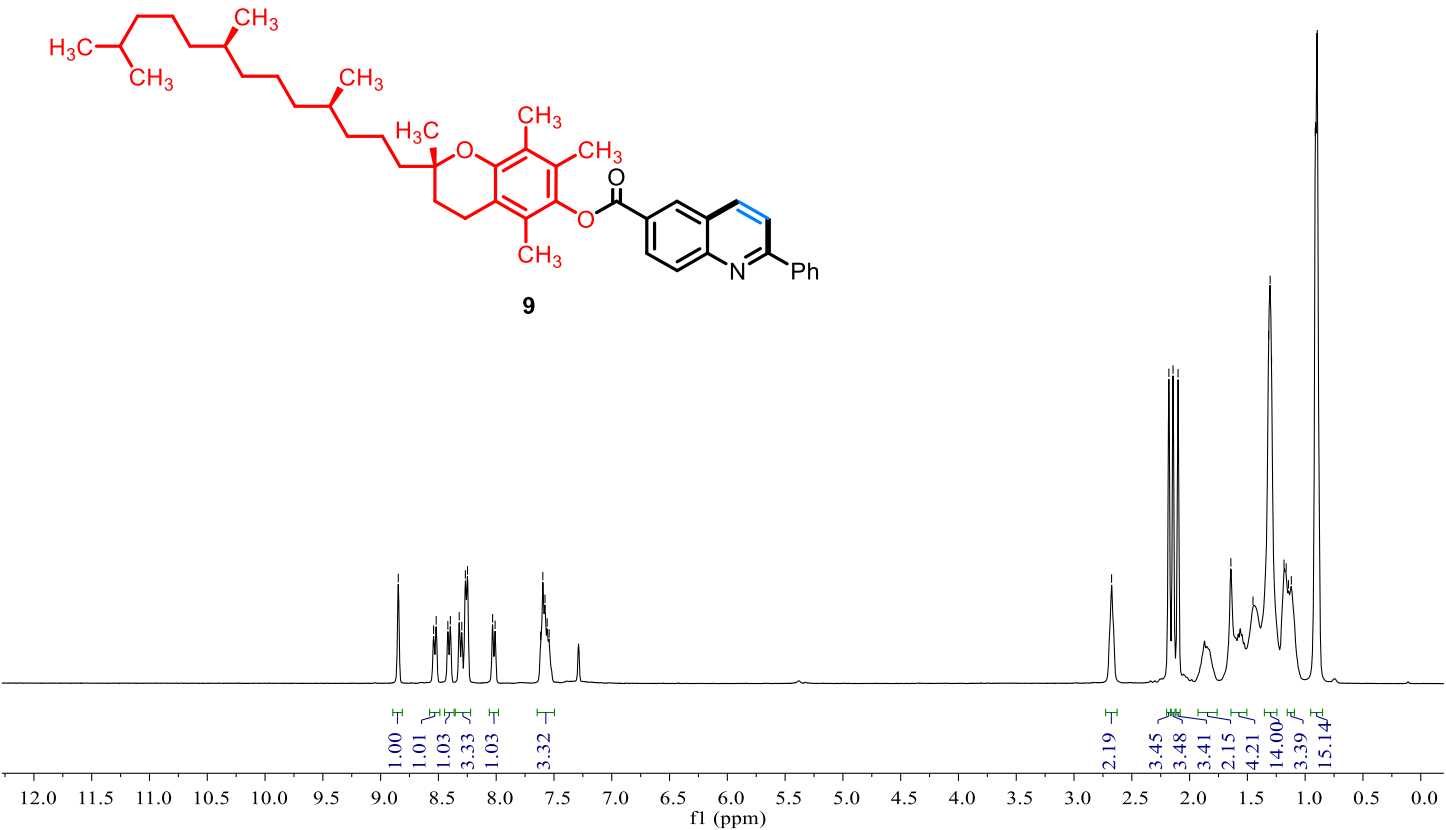

${ }^{13} \mathrm{C}$ NMR of $9\left(100 \mathrm{MHz}, \mathrm{CDCl}_{3}\right)$

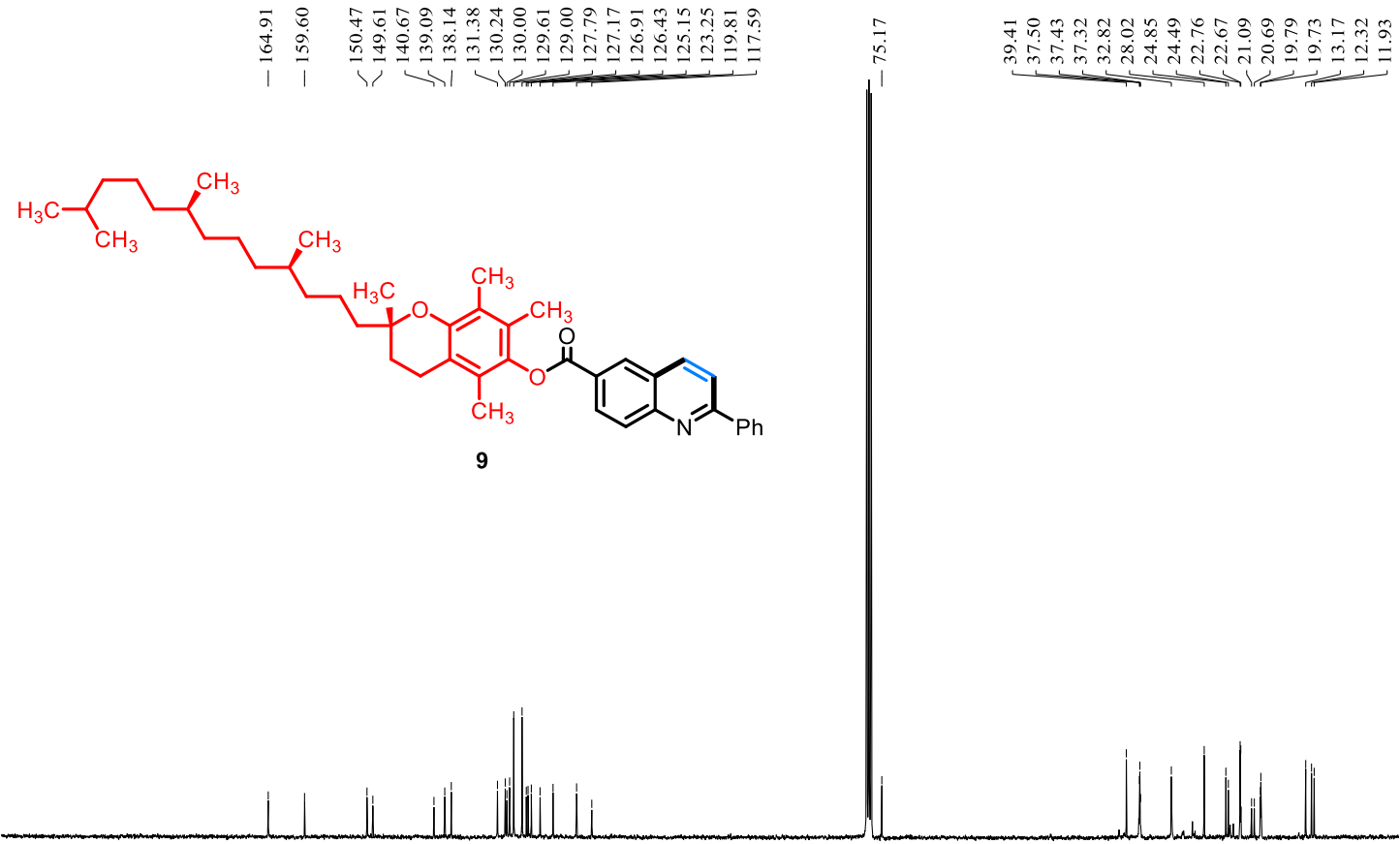

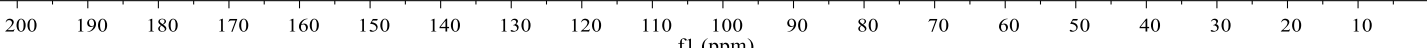

